\title{
Chiral dynamics in form factors, spectral-function sum rules, meson-meson scattering and semilocal duality
}

\author{
Zhi-Hui Guo ${ }^{a, b, *}$ J. A. Oller ${ }^{b, \dagger}$ and J. Ruiz de Elvira ${ }^{c, \ddagger}$ \\ a Department of Physics, Hebei Normal University, 050024 Shijiazhuang, P. R. China. \\ ${ }^{b}$ Departamento de Física, Universidad de Murcia, E-30071 Murcia, Spain. \\ ${ }^{c}$ Departamento de Física Teórica II, Universidad Complutense de Madrid, E-28040 Madrid, Spain.
}

\begin{abstract}
In this work, we perform the one-loop calculation of the scalar and pseudoscalar form factors in the framework of $U(3)$ chiral perturbation theory with explicit tree level exchanges of resonances. The meson-meson scattering calculation from Ref. [1] is extended as well. The spectral functions of the nonet scalar-scalar $(S S)$ and pseudoscalar-pseudoscalar $(P P)$ correlators are constructed by using the corresponding form factors. After fitting the unknown parameters to the scattering data, we discuss the resonance content of the resulting scattering amplitudes. We also study spectral-function sum rules in the $S S$ - $S S, P P-P P$ and $S S-P P$ sectors as well as semilocal duality from scattering. The former relate the scalar and pseudoscalar spectra between themselves while the latter mainly connects the scalar spectrum with the vector one. Finally we investigate these items as a function of $N_{C}$ for $N_{C}>3$. All these results pose strong constraints on the scalar dynamics and spectroscopy that are discussed. They are successfully fulfilled by our meson-meson scattering amplitudes and spectral functions.
\end{abstract}

PACS: $12.39 . \mathrm{Fe}, 11.55 . \mathrm{Hx}, 12.40 . \mathrm{Nn}, 11.15 . \mathrm{Pg}$

Keywords: Chiral perturbation theory, spectral-function sum rules, semilocal duality, $1 / N_{C}$ expansion, scalar resonance dynamics

*guo@um.es

†oller@um.es

¥jacobore@rect.ucm.es 


\section{Contents}

1 Introduction $\quad 3$

2 Chiral Lagrangian $\quad 4$

3 Spectral Functions and Form Factors $\quad 7$

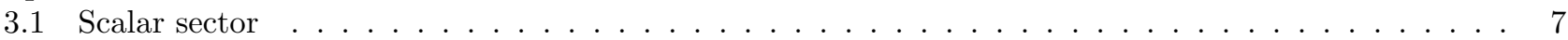

3.2 Pseudoscalar sector . . . . . . . . . . . . . . . . . . . . . . . . . . . . 11

4 Remarks on semilocal duality 12

5 Phenomenological Discussions 13

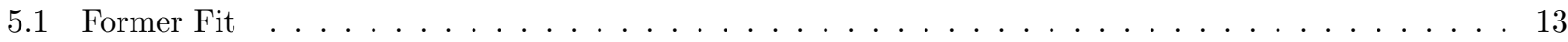

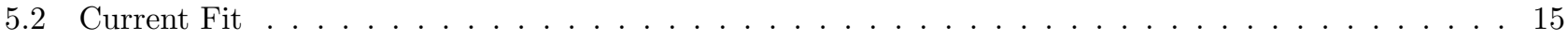

5.3 Phenomenological results of semilocal duality $\ldots \ldots \ldots \ldots \ldots \ldots \ldots$

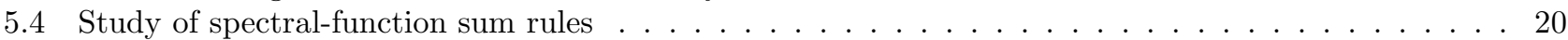

6 Results from the extrapolation of $N_{C} \quad 23$

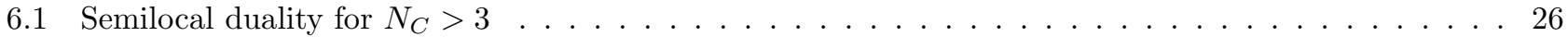

6.2 Resonance pole trajectories with varying $N_{C} \ldots \ldots \ldots \ldots \ldots \ldots \ldots \ldots$

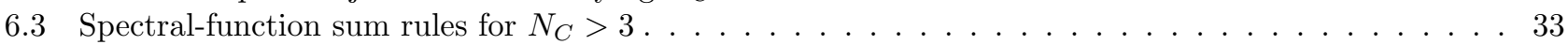

$\begin{array}{llr}7 & \text { Conclusions } & 37\end{array}$

$\begin{array}{lr}\text { A } \text { Masses and mixing parameters } & 38\end{array}$

B Analytic expressions for scalar and pseudoscalar form factors 39

C Tensor resonances in meson-meson scattering $\quad 40$ 


\section{Introduction}

Spectral functions of current-current correlators are interesting objects in hadron physics. They are sensitive to both low and high energy dynamics of QCD, which can be evaluated in two reliable approaches: chiral perturbation theory $(\chi \mathrm{PT})[2,3,4]$ in the low-energy region and the operator product expansion $(\mathrm{OPE})[5,6]$ for the higher one. The celebrated Weinberg sum rules are in fact derived by studying the differences of the vector-vector and axial-vector-axial-vector spectral functions [7], where not only the low and asymptotic energy regions but also the resonance region are considered. After then, great progresses, both on the experimental and theoretical sides [8], have been made along this research line. The vector and axial-vector spectral functions are experimentally measurable quantities, mainly through the $\tau$ decays, due to the noticeable $V-A$ nature of the Standard Model in the energy region well below the $W$ boson mass.

Concerning the scalar and pseudoscalar spectral functions, there are no direct experimental data. However, great efforts have been made on the theoretical sides both in perturbative QCD calculations $[9,10,11]$, which are important to reduce the QCD background in the search of the Higgs particle, and in the nonperturbative QCD region $[3,12,13,14,15,16,17,18,19,20]$. Through the matching between the low and high energy behaviors of the spectral functions, valuable information can be obtained: light quark masses and Cabibbo angle $\left|V_{u s}\right|[9,18,19]$, determination of the low energy constants (LECs) in $\chi \mathrm{PT}[12,13,16,17,21]$ and constraints on resonance parameters $[14,16,17,20,22,23]$.

On the other hand, in the past decade scalar dynamics in the nonperturbative QCD region has been greatly put forward thanks to the combination of $\chi \mathrm{PT}$ and nonperturbative approaches from the $S$-matrix theory [24, 25, 26, $27,28,29,30,31,32,33,34,35]$. One of the main results is the reappearance of the broad $f_{0}(500)$ (traditionally called $\sigma$ ), the lightest resonance in QCD spectrum, after its long absence in the PDG list [36]. Later on the $K_{0}^{*}(800)$ or $\kappa$ resonance in $K \pi$ scattering was also confirmed [36]. Though the mass and width of these broad scalar resonances predicted by different groups are rather consistent among each other, other properties about the resonances in the scalar family are still under a vivid debate (e.g. their nature).

The scalar-scalar $(S S)$ and pseudoscalar-pseudoscalar $(P P)$ correlation functions provide us another theoretical tool to further study the scalar resonances [37], once the phase shifts and inelasticities in meson-meson scattering are well reproduced $[25,26,27,29,30,31,32,33,34,35,38]$. As derived in Refs. $[39,12,13,40,20]$ these correlator functions should fulfill a set of spectral-function sum rules that imply tight constraints to the scalar and pseudoscalar spectra, involving nontrivial relations between them.

We take, and further study, the meson-meson scattering amplitudes of Ref. [1] that are calculated in one-loop $U(3) \chi \mathrm{PT}$ plus the explicit scalar and vector resonance exchanges at tree level within the framework of resonance chiral theory $(\mathrm{R} \chi \mathrm{T})[41]$. As a novelty, we include the contributions from the tree level exchange of pseudoscalar resonances in this work so that a new fit to data is also discussed. The unitarized $U(3) \chi \mathrm{PT}$ amplitudes are more appropriate to incorporate the heavier $f_{0}$ scalar resonances since the channels involving the $\eta$ and $\eta^{\prime}$ mesons can be taken into account (while $S U(3) \chi \mathrm{PT}$ [4] only contains the pure octet $\eta_{8}$ field). As noticed in Ref. [26], further developed later in Refs. [42, 43, 44, 45, 46, 47, 48,34, 1], the study of the resonance pole trajectories with increasing number of colors of QCD, $N_{C}$, is of interest to discern possible natures of the scalar resonances. E.g. a $\bar{q} q$ resonance evolves with a mass $M \sim \mathcal{O}\left(N_{C}^{0}\right)$, while its width decreases as $\Gamma \sim \mathcal{O}\left(N_{C}^{-1}\right)$. For the case of a glueball its mass behaves also as $M \sim \mathcal{O}\left(N_{C}^{0}\right)$ with a width that decreases faster with $N_{C}$ as $\Gamma \sim \mathcal{O}\left(N_{C}^{-2}\right)$ [49, 50]. In this respect $U(3) \chi \mathrm{PT}$ is also more adequate than the $S U(3)$ version to discuss the large $N_{C}$ limit because $U(3) \chi \mathrm{PT}$ includes not only the pseudo-Goldstone octet, as $S U(3)$ does, but also the singlet $\eta_{1}$ that in the chiral limit is the ninth Goldstone boson at large $N_{C}$ [51]. In this way, $U(3) \chi \mathrm{PT}$ has the appropriate low energy degrees of freedom in the chiral limit at large $N_{C}$. Additionally, as stressed in Ref. [1], the $\eta$ meson quickly becomes much lighter with increasing $N_{C}$ [52]. This fact has a strong impact on the dependence with $N_{C}$ of the pole trajectories of the scalar resonances, except for the $f_{0}(500)$, because of its weak couplings to the channels with $\eta$ and $\eta^{\prime}$ [1]. We show below the $N_{C}$ pole trajectories for the different resonances involved in our study from the new fit to data.

Another interesting property of strong interactions that can be used to restrict meson-meson scattering is average or semilocal duality [53]. It allows one to relate the crossed channel dynamics with the $s$-channel one, which supplies another stringent constraint on the resonance properties involved in scattering. In a recent work [54], Peláez et al. have investigated how semilocal duality between the light resonances of QCD and Regge theory can be satisfied in $\pi \pi$ scattering. These authors employ the Inverse Amplitude Method (IAM) $[55,56,57,58]$ to unitarize the $S U(3)$ one-loop and $S U(2)$ two-loop $\chi \mathrm{PT}$ amplitudes. The main conclusion of Ref. [54] is that in the physical case, i.e. 
when $N_{C}=3$, the $f_{0}(500)$ resonance, with its pole position around $440-i 250 \mathrm{MeV}$, plays a crucial role to oppose the vector strength from the $\rho(770)$ resonance, which guarantees the fulfillment of semilocal duality.

Having introduced the main research topics considered here for the sake of clarity we summarize our work and briefly comment on the main points and results obtained by considering these topics. A complete one-loop calculation of the scalar and pseudoscalar form factors within $U(3)$ unitary $\chi \mathrm{PT}$, including the tree-level exchange of scalar, pseudoscalar and vector resonances, is undertaken. The spectral functions of the $S S$ and $P P$ two-point correlators are constructed by using the resulting scalar and pseudoscalar form factors, respectively, which are unitarized for the case of the scalar ones. After updating the fit in Ref. [1], which is also extended by including the explicit exchange of pseudoscalar resonances, we study the resonance spectroscopy, quadratic pion scalar radius, the fulfillment of semilocal duality and the spectral function sum rules in the $S S-S S, P P-P P$ and $S S-P P$ cases, which are remarkably well satisfied simultaneously. We show that it is important to take under consideration the high energy constraint for the coupling of the vector resonances to pseudo-Goldstone bosons, in order to keep semilocal duality when varying $N_{C}$. An interesting interplay between different resonances when studying the $N_{C}$ evolution of semilocal duality and the spectral function sum rules is revealed. In the former case the scalar and vector spectra appear tightly related and in the latter one the same can be stated for the scalar and pseudoscalar ones. This issue is closely connected with the evolution of the resonance pole position with varying $N_{C}$ of the pseudoscalar, vector and scalar resonances.

In this respect, for larger $N_{C}$, the authors of Ref. [54] revealed that a $\bar{q} q$ subdominant component for the $f_{0}(500)$, with a mass around $1 \mathrm{GeV}$ and obtained for some sets of LECs in both one-loop and two-loop calculations, is needed in order to fulfill semilocal duality. For those solutions, the $f_{0}(500)$ pole moves away from the $400-700 \mathrm{MeV}$ region of the real axis, but it turns around moving back towards the real axis above $1 \mathrm{GeV}$ as $N_{C}$ becomes larger than 8 or so. The interpretation of these results was studied preliminarily in Ref. [59], where the $f_{0}(500)$ is considered as a combination of several states and it was found that the weight of the subdominant $\bar{q} q$ component in the $f_{0}(500)$ grows with increasing $N_{C}$. Immediately, one can ask a question: apart from the $f_{0}(500)$ resonance, which is only marginally contributed by this $\bar{q} q$ component, are there any other more obvious effects from the $\bar{q} q$ seed at $N_{C}=3$ ? If there are, what kind of role do these effects play in the fulfillment of semilocal duality?

Concerning the first question, we provide here, confirming Refs. [26, 1], a scenario for an affirmative answer. A bare singlet scalar resonance $S_{1}$, with its bare mass around $1 \mathrm{GeV}$, is found significant [1] to reproduce the $\pi \pi$ and $K \bar{K}$ scattering data around the $f_{0}(980)$ energy region and, thus, becomes an important part of the $f_{0}(980)$ resonance in the physical case, i.e. at $N_{C}=3$. When increasing $N_{C}$, the physical $f_{0}(980)$ resonance, gradually evolves to the singlet $S_{1}$, which acts like the role of the $\bar{q} q$ seed described in Refs. [54, 59]. Apparently, the scenario that we provide now is different from that in Ref. [54], since in the latter reference the signal of the $f_{0}(980)$ resonance gradually disappears when increasing $N_{C} .{ }^{1}$ Regarding the second question raised in the previous paragraph we find here that its role for fulfilling the requirements of semilocal duality becomes more important with increasing $N_{C}$, being only of little importance at $N_{C}=3$. This behavior is in qualitative agreement with the results of [54].

In addition to the results presented in our short letter [37], we show our theoretical calculations in detail and discuss more phenomenological materials in this work, such as the fit results, resonance pole positions and their coupling strengths, $N_{C}$ trajectories for all the relevant resonances and so on. The contents of this article are organized as follows. Section 2 is devoted to the introduction of the relevant chiral Lagrangian. In Sections 3 and 4 , we present the details of the calculations of the spectral functions and the quantities to measure the degree of fulfillment of semilocal duality, respectively. The phenomenological discussions are given in Section 5, which include the fit of unknown parameters to the experimental data and its consequences on the form factors, spectral functions, spectral-function sum rules and semilocal duality. Section 6 is then devoted to the study of the $N_{C}$ evolution of these quantities. Finally, we conclude in Section 7.

\section{Chiral Lagrangian}

The theoretical framework that we use in the present work is $U(3) \chi \mathrm{PT}[60,61]$, the tree level resonance exchanges from chiral invariant Lagrangians [41] and unitary $\chi \mathrm{PT}[26,62]$. The $U(3) \otimes U(3)$ chiral symmetry in $u, d, s$ massless QCD is broken because of quantum effects that violate the conservation of the singlet axial-vector current by the $U_{A}(1)$ anomaly $[63,64,65]$. As a result the pseudoscalar $\eta^{\prime}$ is not a pseudo-Goldstone boson [66]. Nevertheless,

\footnotetext{
${ }^{1}$ See the left panel of Fig.1 in Ref. [54]. It is verified that the peak around $1 \mathrm{GeV}^{2}$ finally disappears with larger values of $N_{C}$.
} 
from the large $N_{C}$ QCD point of view the quark loop responsible for the $U_{A}(1)$ anomaly [63] is $1 / N_{C}$ suppressed. In this way, the $\eta^{\prime}$ becomes the ninth pseudo-Goldstone boson in the large $N_{C}$ limit [51]. In large $N_{C}$ QCD the singlet field $\eta_{1}$ can be conveniently incorporated into the effective field theory by enlarging the number of degrees of freedom of the theory from an octet to a nonet of pseudo-Goldstone bosons, which is usually called $U(3) \chi \mathrm{PT}$ [60]. In this theory there are three expansion parameters: momentum, quark masses and $1 / N_{C}$, giving rise to a joint triple expansion $\delta \sim p^{2} \sim m_{q} \sim 1 / N_{C}$. We briefly recapitulate the relevant chiral Lagrangians for our work.

The leading order Lagrangian in $U(3) \chi \mathrm{PT}$ reads [60]

$$
\mathcal{L}_{\chi}=\frac{F^{2}}{4}\left\langle u_{\mu} u^{\mu}\right\rangle+\frac{F^{2}}{4}\langle\chi+\rangle+\frac{F^{2}}{3} M_{0}^{2} \ln ^{2} \operatorname{det} u
$$

where $\langle\ldots\rangle$ stands for the trace in flavor space and the last operator is the $U_{A}(1)$ anomaly term that gives rise to the singlet $\eta_{1}$ mass. The definitions for the chiral building blocks are

$$
\begin{aligned}
u_{\mu} & =i u^{+} D_{\mu} U u^{+}, \\
\chi_{ \pm} & =u^{+} \chi u^{+} \pm u \chi^{+} u, \\
U & =u^{2}=e^{i \frac{\sqrt{2} \Phi}{F}}, \\
D_{\mu} U & =\partial_{\mu} U-i r_{\mu} U+i U l_{\mu}, \\
\chi & =2 B(s+i p),
\end{aligned}
$$

where $r_{\mu}, l_{\mu}, s, p$ are external sources [3] and the pseudo-Goldstone bosons are collected in the matrix

$$
\Phi=\left(\begin{array}{ccc}
\frac{1}{\sqrt{2}} \pi^{0}+\frac{1}{\sqrt{6}} \eta_{8}+\frac{1}{\sqrt{3}} \eta_{1} & \pi^{+} & K^{+} \\
\pi^{-} & \frac{-1}{\sqrt{2}} \pi^{0}+\frac{1}{\sqrt{6}} \eta_{8}+\frac{1}{\sqrt{3}} \eta_{1} & K^{0} \\
K^{-} & \bar{K}^{0} & \frac{-2}{\sqrt{6}} \eta_{8}+\frac{1}{\sqrt{3}} \eta_{1}
\end{array}\right) .
$$

The axial decay constant of the pseudo-Goldstone bosons in the simultaneous chiral and large $N_{C}$ limit is denoted by $F$. In the same limit the parameter $B$ is related to the quark condensate through $\left\langle 0\left|\bar{q}^{i} q^{j}\right| 0\right\rangle=-F^{2} B \delta^{i j}$. We do not consider isospin $(I)$ breaking effects throughout.

In the present work, we exploit the assumption on the resonance saturation of the LECs [41, 67], so that, instead of local chiral terms contributing to meson-meson scattering, we take the tree level exchanges of the scalar, pseudoscalar and vector resonances. In this way we keep all local contributions to meson-meson scattering up to and including $\mathcal{O}\left(\delta^{3}\right)$, while also generating higher order ones. We also include the one-loop contributions that count one order higher in $\delta$, as calculated in Ref. [1].

The terms that describe the interactions between scalar resonances and pseudo-Goldstone bosons in $\mathrm{R} \chi \mathrm{T}$ $\operatorname{are}^{2}[41]$

$$
\mathcal{L}_{S}=c_{d}\left\langle S_{8} u_{\mu} u^{\mu}\right\rangle+c_{m}\left\langle S_{8} \chi_{+}\right\rangle+\widetilde{c}_{d} S_{1}\left\langle u_{\mu} u^{\mu}\right\rangle+\widetilde{c}_{m} S_{1}\left\langle\chi_{+}\right\rangle
$$

and for the vector resonances it reads

$$
\mathcal{L}_{V}=\frac{i G_{V}}{2 \sqrt{2}}\left\langle V_{\mu \nu}\left[u^{\mu}, u^{\nu}\right]\right\rangle
$$

where the anti-symmetric tensor formalism is used to describe the vector resonances [41]. In addition, we also include the pseudoscalar resonances in this work, which are not considered in Ref. [1]. The relevant Lagrangian reads [41]

$$
\mathcal{L}_{P}=i d_{m}\left\langle P_{8} \chi_{-}\right\rangle+i \widetilde{d}_{m} P_{1}\left\langle\chi_{-}\right\rangle
$$

\footnotetext{
${ }^{2}$ The terms $\hat{c}_{d}\left\langle S_{9} u_{\mu}\right\rangle\left\langle u_{\mu}\right\rangle+\hat{c}_{m} S_{1} \ln ^{2} \operatorname{det} u$ were also introduced in Ref. [1] but found phenomenologically irrelevant. This is in agreement with the fact that from the exchange of scalar resonances they start to generate tree level meson-meson contributions that are of higher order, $\mathcal{O}\left(\delta^{4}\right)$.
} 
The corresponding kinetic terms for the resonance fields are [41]

$$
\begin{gathered}
\mathcal{L}_{\text {kin }}^{V}=-\frac{1}{2}\left\langle\nabla^{\lambda} V_{\lambda \mu} \nabla_{\nu} V^{\nu \mu}-\frac{1}{2} M_{V}^{2} V_{\mu \nu} V^{\mu \nu}\right\rangle, \\
\mathcal{L}_{\text {kin }}^{R}=\frac{1}{2}\left\langle\nabla^{\mu} R_{8} \nabla_{\mu} R_{8}-M_{R_{8}}^{2} R_{8}^{2}\right\rangle+\frac{1}{2}\left(\partial^{\mu} R_{1} \partial_{\mu} R_{1}-M_{R_{1}}^{2} R_{1}^{2}\right),
\end{gathered}
$$

where the symbol $R$ refers to either scalar or pseudoscalar resonances.

One should notice that the operators appearing in Eq. (6) cause mixing terms proportional to the quark masses between the pseudoscalar resonances and the pseudo-Goldstone bosons. Though it is not a problem to consider this effect in the calculation of Feynman diagrams, it would be convenient to eliminate the mixing terms at the Lagrangian level. Indeed this can be accomplished by performing the following field redefinition in a chiral covariant way:

$$
\begin{aligned}
& P_{8} \rightarrow \bar{P}_{8}+i \frac{d_{m}}{M_{P_{8}}^{2}}\left(\chi_{-}-\frac{1}{3}\left\langle\chi_{-}\right\rangle I_{3 \times 3}\right), \\
& P_{1} \rightarrow \bar{P}_{1}+i \frac{\widetilde{d}_{m}}{M_{P_{1}}^{2}}\left\langle\chi_{-}\right\rangle,
\end{aligned}
$$

with $I_{3 \times 3}$ the unit $3 \times 3$ matrix. Substituting Eq. (9) into Eqs. (6) and (8), we have the new Lagrangian

$$
\begin{aligned}
\mathcal{L}_{\bar{P}} & =\frac{1}{2}\left\langle\nabla^{\mu} \bar{P}_{8} \nabla_{\mu} \bar{P}_{8}-M_{P_{8}}^{2} \bar{P}_{8}^{2}\right\rangle+\frac{1}{2}\left(\partial^{\mu} \bar{P}_{1} \partial_{\mu} \bar{P}_{1}-M_{P_{1}}^{2} \bar{P}_{1}^{2}\right) \\
& +i \frac{d_{m}}{M_{P_{8}}^{2}}\left\langle\nabla_{\mu} \bar{P}_{8} \nabla^{\mu} \chi_{-}\right\rangle+i \frac{\widetilde{d}_{m}}{M_{P_{1}}^{2}} \nabla_{\mu} \bar{P}_{1} \nabla^{\mu}\left\langle\chi_{-}\right\rangle \\
& -\frac{d_{m}^{2}}{2 M_{P_{8}}^{2}}\left\langle\chi_{-} \chi_{-}\right\rangle+\left(\frac{d_{m}^{2}}{6 M_{P_{8}}^{2}}-\frac{\widetilde{d}_{m}^{2}}{2 M_{P_{1}}^{2}}\right)\left\langle\chi_{-}\right\rangle\left\langle\chi_{-}\right\rangle+\cdots,
\end{aligned}
$$

where the omitted terms, represented by the dots in the last line, denote the local chiral operators that describe the interactions between the pseudo-Goldstone bosons at $\mathcal{O}\left(p^{6}\right)$ that we disregard throughout. We point out that the above procedure only eliminates the mixing terms between the pseudoscalar resonances and pseudo-Goldstone bosons that are linearly proportional to quark masses. The new mixing terms with higher power of quark masses that arise through the operators in the second line of Eq. (10) are $\mathcal{O}\left(p^{6}\right)$ contributions, which are then not considered in this work. It is interesting to point out that after the field redefinition of Eq. (9), two new operators describing interactions between pseudo-Goldstone bosons appear, i.e. the ones in the third line of Eq. (10). They coincide with the $\mathcal{O}\left(p^{4}\right)$ terms that result from the integration of the pseudoscalar resonances [41].

We remind that the nature of the pseudoscalar resonances is still a somewhat debated issue and also that typically the parameters of those resonances are not determined accurately [36]. In Ref. [68] the pseudoscalar resonances are generated dynamically due to the interactions between the scalar resonances and the pseudo-Goldstone bosons, instead of introducing them as basic degrees of freedom at the Lagrangian level. In order to compensate for the uncertainties arising from the not well settled properties of the pseudoscalar resonances, as well as from the simple tree level exchanges with bare propagators that we take here to describe them (neglecting more involved contributions as those pointed out in Ref. [68]), we introduce an $L_{8}$-like operator [4] in our study

$$
\frac{\delta L_{8}}{2}\left\langle\chi_{+} \chi_{+}+\chi_{-} \chi_{-}\right\rangle
$$

The reason behind is that only $L_{8}$ and $L_{7}$ could receive contributions at the $\mathcal{O}\left(p^{4}\right)$ level after the integration of the pseudoscalar resonances from Eqs. (6) and (8). Nevertheless the $L_{7}$ term vanishes if one further imposes the large $N_{C}$ relations for the pseudoscalar resonances (as we take here)

$$
\begin{aligned}
\tilde{d}_{m} & =\frac{d_{m}}{\sqrt{3}}, \\
M_{P_{1}} & =M_{P_{8}} .
\end{aligned}
$$


We stress that $\delta L_{8}$ is different from $L_{8}$ in the $\chi \mathrm{PT}$ Lagrangian [4] and their relation can be written as

$$
L_{8}^{\chi \mathrm{PT}}=L_{8}^{\text {Resonances }}+\delta L_{8} .
$$

So that we interpret $\delta L_{8}$ as the contributions from some remnant pieces that are sub-leading in $1 / N_{C}$, minding that the leading $N_{C}$ contributions are already included in the resonance part.

Finally, there are two relevant terms at $\mathcal{O}(\delta)$ that only incorporate pseudo-Goldstone bosons [69] and cannot be generated from the exchange of the explicit resonance fields discussed above. These contributions are:

$$
\begin{aligned}
\mathcal{L}_{\Lambda} & =\Lambda_{1} \frac{F^{2}}{12} D_{\mu} \psi D^{\mu} \psi-i \Lambda_{2} \frac{F^{2}}{12}\left\langle U^{+} \chi-\chi^{+} U\right\rangle \psi, \\
\psi & =-i \ln \operatorname{det} U \\
D_{\mu} \psi & =\partial_{\mu} \psi-2\left\langle a_{\mu}\right\rangle,
\end{aligned}
$$

with $a_{\mu}=\left(r_{\mu}-l_{\mu}\right) / 2$. As we commented in Ref. [1] the $\Lambda_{1}$ parameter only affects the calculation of the masses, scattering amplitudes and also the scalar and pseudoscalar form factors in an indirect way, i.e. through the renormalization of the $\eta_{1}$ field and its effect in the global fit is tiny. We explicitly check that if we include $\Lambda_{1}$ in our discussion, the fitted value for this parameter approaches to zero. So that this operator is not considered in the following.

For the remaining definitions of the basic chiral building blocks, the reader is referred to Ref.[1] and references therein for further details.

\section{Spectral Functions and Form Factors}

The two-point correlation function is defined as

$$
\delta^{a b} \Pi_{R}\left(p^{2}\right)=i \int d^{4} x e^{i p \cdot x}<0\left|T\left[R^{a}(x) R^{b}(0)\right]\right| 0>,
$$

where the scalar and pseudoscalar densities correspond to $R^{a} \equiv S^{a}=\bar{q} \lambda_{a} q$ and $R^{a} \equiv P^{a}=i \bar{q} \gamma_{5} \lambda_{a} q$, in order, with $\lambda_{a}$ the Gell-Mann matrices for $a=1, \ldots, 8$ and $\lambda_{0}=I_{3 \times 3} \sqrt{2 / 3}$ for $a=0$. The spectral-function sum rule in the chiral limit can be then represented as

$$
\int_{0}^{\infty}\left[\operatorname{Im} \Pi_{R}(s)-\operatorname{Im} \Pi_{R^{\prime}}(s)\right] d s=0,
$$

where $R$ and $R^{\prime}$ are different scalar or pseudoscalar densities mentioned above. The imaginary part of the two-point correlation function $\operatorname{Im} \Pi_{R}$, also called the spectral function, is one of the key quantities that we calculate in this work.

\subsection{Scalar sector}

The scalar form factor of a pseudo-Goldstone boson pair $P Q$ is defined as

$$
F_{P Q}^{a}(s)=\frac{1}{B}\left\langle 0\left|\bar{q} \lambda_{a} q\right| P Q\right\rangle .
$$

In the present work, we focus on the chiral dynamics for the components with $a=0,1,2,3,8$, which preserve the strangeness. By imposing the isospin symmetry, only three of the five components are independent. We take the neutral ones, i.e. $a=0,8,3$, which correspond to the singlet and $I=0,1$ octet $S U(3)$ densities, respectively. The scalar form factors with components $a=4,5,6,7$ correspond to the strangeness changing ones with $I=\frac{1}{2}$ and $\frac{3}{2}$, that were studied in [23].

The scalar spectral function is related to the scalar form factors through

$$
\operatorname{Im} \Pi_{S^{a}}(s)=\sum_{i} \rho_{i}(s)\left|F_{i}^{a}(s)\right|^{2} \theta\left(s-s_{i}^{\mathrm{th}}\right),
$$


where $\theta(x)$ is the Heaviside step function and the sum on $i$ extends over the different pseudo-Goldstone boson pairs. In addition, $s$ is the energy squared in the center of mass frame, $s_{i}^{\text {th }}=\left(m_{A}+m_{B}\right)^{2}$ is the threshold of the $i_{\text {th }}$ channel and $m_{A}, m_{B}$ are the masses of the corresponding two particles. In the previous equation only twobody intermediate states are considered, the same ones as taken in Ref. [1] to study meson-meson scattering. We point out that the unitarized scalar form factors, among others, include the contributions of the single resonance exchanges to the spectral functions. We proof below, when discussing the evolution with $N_{C}$, that the two-point correlator in our analysis reduces to the single resonance exchange diagram at large $N_{C}$ [70].

The phase space factor for the $i_{\text {th }}$ channel in Eq. (19) is

$$
\rho_{i}(s)=\frac{\sqrt{\left[s-\left(m_{A}+m_{B}\right)^{2}\right]\left[s-\left(m_{A}-m_{B}\right)^{2}\right]}}{16 \pi s}=\frac{q_{i}}{8 \pi \sqrt{s}},
$$

being $q_{i}$ the three momentum in center of mass frame.

For the isoscalar case, there are five two-particle intermediate states made by a pseudo-Goldstone pair in $U(3)$ $\chi \mathrm{PT}$, namely, $\pi \pi, K \bar{K}, \eta \eta, \eta \eta^{\prime}$ and $\eta^{\prime} \eta^{\prime}$. For the isovector case, there are three channels: $\pi \eta, K \bar{K}$ and $\pi \eta^{\prime}$. The two-particle states with well defined isospin for the isoscalar case read

$$
\begin{aligned}
|\pi \pi\rangle_{I=0} & =-\frac{1}{\sqrt{2}} \frac{\left|\pi^{+} \pi^{-}\right\rangle+\left|\pi^{-} \pi^{+}\right\rangle+\left|\pi^{0} \pi^{0}\right\rangle}{\sqrt{3}} \\
|K \bar{K}\rangle_{I=0} & =-\frac{\left|K^{+} K^{-}\right\rangle+\left|K^{0} \bar{K}^{0}\right\rangle}{\sqrt{2}}, \\
|\eta \eta\rangle_{I=0} & =\frac{|\eta \eta\rangle}{\sqrt{2}}, \\
\left|\eta \eta^{\prime}\right\rangle_{I=0} & =\left|\eta \eta^{\prime}\right\rangle \\
\left|\eta^{\prime} \eta^{\prime}\right\rangle_{I=0} & =\frac{\left|\eta^{\prime} \eta^{\prime}\right\rangle}{\sqrt{2}} .
\end{aligned}
$$

For the isovector case they are

$$
\begin{aligned}
|\pi \eta\rangle_{I=1} & =|\pi \eta\rangle \\
|K \bar{K}\rangle_{I=1} & =-\frac{\left|K^{+} K^{-}\right\rangle-\left|K^{0} \bar{K}^{0}\right\rangle}{\sqrt{2}} \\
\left|\pi \eta^{\prime}\right\rangle_{I=1} & =\left|\pi \eta^{\prime}\right\rangle .
\end{aligned}
$$

We point out that the so-called unitary normalization for the identical particles, as proposed in Ref. [25], has been used in Eq. (21) for the $\pi \pi, \eta \eta$ and $\eta^{\prime} \eta^{\prime}$ states. In this way, the normalization that we employ in this work coincides with the one used in [1]. We can then construct the unitarized form factors by using the partial wave scattering amplitudes calculated in [1] without any adjustment in the normalization.

The perturbative calculation of the scalar form factors of the pseudo-Goldstone pairs at the one-loop level consists of evaluating the Feynman graphs shown in Fig. 1. As we have done for the scattering in Ref. [1], the contributions beyond the leading order to the form factors are calculated in terms of $\bar{\eta}$ and $\bar{\eta}^{\prime}$ fields that result by diagonalizing the fields $\eta_{8}$ and $\eta_{1}$ at leading order from Eq. (1)

$$
\begin{aligned}
& \eta_{8}=c_{\theta} \bar{\eta}+s_{\theta} \bar{\eta}^{\prime}, \\
& \eta_{1}=-s_{\theta} \bar{\eta}+c_{\theta} \bar{\eta}^{\prime},
\end{aligned}
$$

with $c_{\theta}=\cos \theta$ and $s_{\theta}=\sin \theta$. Compared to the basis with the fields $\eta_{8}$ and $\eta_{1}$, the use of the fields $\bar{\eta}$ and $\bar{\eta}^{\prime}$ allows us to include the relevant Feynman diagrams conveniently in the calculation. As discussed in Ref. [1] we avoid in this way having to include an arbitrary number of insertions of the leading order $\eta_{8}-\eta_{1}$ mixing. As a result, one can consider the $\eta$ and $\eta^{\prime}$ mixing effects in the form factors order by order in the $\delta$ counting scheme.

There are several sources that contribute to diagram (f) in Fig. $1: \bar{\eta}-\bar{\eta}^{\prime}$ mixing (notice that it is different from $\eta_{1}-\eta_{8}$ mixing [1], as just discussed), and the local contributions from the last two terms in Eq. (10) and the 


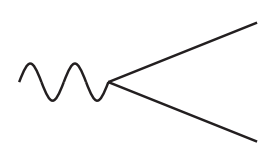

(a)

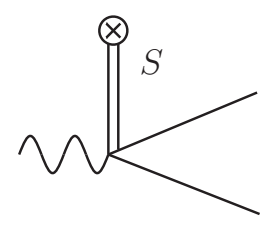

(d)

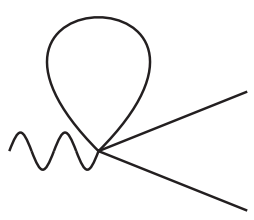

(b)

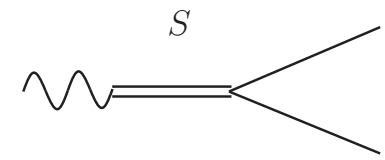

(e)

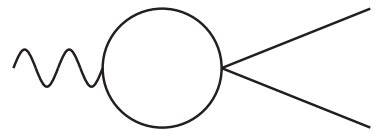

(c)

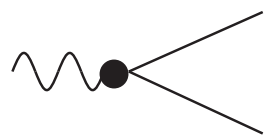

(f)

Figure 1: Feynman diagrams for the calculation of the scalar form factor in one-loop $U(3) \chi \mathrm{PT}$. The wavy line denotes the scalar external source. The cross in diagram (d) indicates the coupling between the scalar resonance and the vacuum. The filled circle in diagram (f) corresponds to the vertices that only involve the pseudo-Goldstone bosons and are beyond leading order from Eq. (1). The different terms that contribute to diagram (f) are explained in detail in the text.

operators in Eqs. (11) and (15). The contributions from the $\bar{\eta}-\bar{\eta}^{\prime}$ mixing to the form factors are calculated in the same way as for the calculation of scattering amplitudes in Ref. [1]. First, we substitute Eq. (16) of Ref. [1], which parameterizes the relations between $\bar{\eta}, \bar{\eta}^{\prime}$ and the physical states $\eta, \eta^{\prime}$, into the leading order Lagrangian Eq. (1). Afterwards, we calculate the form factors in terms of the physical states $\eta$ and $\eta^{\prime}$. Due to the inclusion of the pseudoscalar resonances and the operator in Eq. (11), which contribute to the $\bar{\eta}-\bar{\eta}^{\prime}$ mixing parameters $\delta_{i}$ in Eq. (14) of Ref. [1], we present the updated results in Appendix A. In addition, the wave function renormalization of the pseudo-Goldstone bosons, which has been calculated in Ref. [1], also contributes to the scalar form factors. The explicit expressions for the scalar form factors are given in Appendix B.

In $U(3) \chi \mathrm{PT}$ the resummation of the unitarity chiral loops is essential because of the large mass of the $s$ quark and the large anomaly mass in Eq. (1). Then, in many kinematical regions the resulting pseudo-Goldstone thresholds are much larger than the three-momenta involved and this enhances the two-pseudo-Goldstone reducible loop contributions [71]. In addition, we are also interested in the chiral dynamics involving the resonance region where the unitarity upper bound in partial waves could be easily found to be saturated. So that it is not meaningful to treat unitarity in a perturbative way as in $\chi \mathrm{PT}$. As a result, one must resum the right-hand cut that stems from unitarity and for that we employ Unitary $\chi \mathrm{PT}(\mathrm{U} \chi \mathrm{PT})$, both for calculating the meson-meson partial wave amplitudes $[26,22,1]$ and the scalar form factors $[72,73,74]$.

The unitarization method that we use to resum the unitarity cut for the scalar form factors was developed in Ref. [73, 72]. It is based on the $N / D$ method and was first applied to meson-meson scattering in Ref. [26]. For completeness, we recapitulate the essentials of this method here.

In the case of two-particle intermediate states, the absorptive part of the form factor obeys the relation

$$
\operatorname{Im} F_{j}^{I}(s)=\sum_{k=1}^{Z} T_{j k}^{I J}(s)^{*} \rho_{k}(s) F_{k}^{I}(s),
$$

where $T^{I J}(s)$ denotes the partial wave scattering amplitudes with definite isospin number $I$ and angular momentum $J$ and $Z$ is the number of channels with the same quantum numbers $I J$. The $T$-matrix $T^{00}(s)$, relevant to the scalar form factors with $a=0,8$, is a $5 \times 5$ matrix and $T^{10}(s)$, relevant to $a=3$, is a $3 \times 3$ matrix. Both of them are studied in detail in Ref. [1]. Nevertheless due to the fact that now we also include the pseudoscalar resonances 
and the $\delta L_{8}$ operator, we need to consider their contributions in the scattering amplitudes as well. The effect from the pseudoscalar resonances can be easily included, since after the field redefinition the only relevant terms are the last two operators in Eq. (10). The pertinent expressions for the perturbative scattering amplitudes from $U(3)$ $\chi \mathrm{PT}$ including explicit exchanges of scalar and vector resonances [1] is given in Ref. [75]. The new contributions from the $\delta L_{8}$ operator and pseudoscalar resonances (as well as from tensor resonances, introduced below) are given in [76], together with the expressions for the form factors.

Following the method elaborated in Refs. [72, 73], see Ref. [74] for a simplified discussion, the unitarized scalar form factor can be cast as

$$
F^{I}(s)=\left[1+N^{I J}(s) g^{I J}(s)\right]^{-1} R^{I}(s),
$$

where

$$
\begin{gathered}
N^{I J}(s)=T^{I J}(s)^{(2)+\text { Res+Loop }}+T^{I J}(s)^{(2)} g^{I J}(s) T^{I J}(s)^{(2)}, \\
R^{I}(s)=F^{I}(s)^{(2)+\text { Res+Loop }}+N^{I J}(s)^{(2)} g^{I J}(s) F^{I}(s)^{(2)} .
\end{gathered}
$$

The matrix $N^{I J}(s)$ contains the crossed-channel cuts from the meson-meson scattering and the bare resonance poles. It was calculated in Ref. [1], and extended here by including the pseudoscalar resonance exchanges and the $\delta L_{8}$ operator. The superscripts (2), Res and Loop in Eq. (26) denote the perturbative calculations from the tree level result using the leading order Lagrangian Eq. (1), resonance contributions (also including the operators in Eqs. (11) and (15)) and pseudo-Goldstone loops, respectively. On the other hand, $R^{I}(s)$ is a vector with $Z$ rows constructed from the $U(3) \chi \mathrm{PT}$ form factors similarly as $N^{I J}(s)$ is calculated for scattering. The vector $R^{I}(s)$ does not contain any cut singularity and is real $[72,73]$.

The matrix $g^{I J}(s)$ in Eq. (26) is a diagonal $Z \times Z$ matrix, with its $i_{\text {th }}$ non-vanishing matrix element given by

$$
\begin{aligned}
16 \pi^{2} g^{I J}(s)_{i} & =a_{S L}(\mu)+\log \frac{m_{B}^{2}}{\mu^{2}}-x_{+} \log \frac{x_{+}-1}{x_{+}}-x_{-} \log \frac{x_{-}-1}{x_{-}}, \\
x_{ \pm} & =\frac{s+m_{A}^{2}-m_{B}^{2}}{2 s} \pm \frac{1}{2 s} \sqrt{-4 s\left(m_{A}^{2}-i 0^{+}\right)+\left(s+m_{A}^{2}-m_{B}^{2}\right)^{2}},
\end{aligned}
$$

where $a_{S L}(\mu)$ is a subtraction constant. The matrix $g^{I J}(s)$ collects the discontinuity caused by the two-particle intermediate states along the right hand cut and plays a key role in the $N / D$ unitarization approach. We refer to Section IV of Ref. [1], and references therein, for detailed discussions on the calculation of the scattering $T$-matrix. The latter is given by a similar expression to Eq. (25) in terms of $N^{I J}(s)$ and $g^{I J}(s)$,

$$
T^{I J}(s)=\left[1+N^{I J}(s) g^{I J}(s)\right]^{-1} N^{I J}(s) .
$$

Before ending this section, we introduce the scalar form factors in the quark flavor basis instead of in the singletoctet flavor basis. There are two kinds of isoscalar scalar densities in the quark flavor basis: $\bar{u} u+\bar{d} d$ and $\bar{s} s$. The relations between the form factors in the two different bases read

$$
\begin{aligned}
F^{\bar{u} u+\bar{d} d} & =\frac{1}{\sqrt{3}} F^{a=8}+\sqrt{\frac{2}{3}} F^{a=0}, \\
F^{\bar{s} s} & =-\frac{1}{\sqrt{3}} F^{a=8}+\frac{1}{\sqrt{6}} F^{a=0} .
\end{aligned}
$$

The pion scalar radius $\left\langle r^{2}\right\rangle_{S}^{\pi}$, an important low energy observable, is defined through the low energy Taylor expansion of the pion scalar form factor in the quark flavor basis $[77,78]$

$$
F_{\pi \pi}^{\bar{u} u+\bar{d} d}(s)=F_{\pi \pi}^{\bar{u} u+\bar{d} d}(0)\left[1+\frac{1}{6}\left\langle r^{2}\right\rangle_{S}^{\pi} s+\ldots\right],
$$

with

$$
m_{\pi}^{2} F_{\pi \pi}^{\bar{u} u+\bar{d} d}(s) \equiv 2 B m\langle 0|\bar{u} u+\bar{d} d| \pi \pi\rangle_{I=0} .
$$

In the above equation, $m$ stands for the up or down quark mass (we ignore isospin breaking) so that $2 B m=\bar{m}_{\pi}^{2}$, with $\bar{m}_{\pi}$ the leading order pion mass from Eq. (1). The relation between the physical mass square of the pion, $m_{\pi}^{2}$, and the leading order one, $\bar{m}_{\pi}^{2}$, was given in the Appendix of Ref. [1]. The updated version that includes the pseudoscalar resonances and the $\delta L_{8}$ effect is collected in Appendix A. 


\subsection{Pseudoscalar sector}

For the pseudoscalar spectral function, the leading order contribution is due to the single pseudo-Goldstone boson exchange. The next non-vanishing contribution from the pure pseudo-Goldstone system requires at least three intermediate mesons, which belongs to a two-loop calculation that is beyond the scope of our current discussion. In order to take into account the chiral dynamics above $1 \mathrm{GeV}$ in the pseudoscalar spectral function, we include the pseudoscalar resonance exchanges explicitly making use of the chiral invariant Lagrangian, Eq. (10). In light of the results of the work [68], where the pseudoscalar resonances were dynamically generated through the scattering of the scalar resonances and the pseudo-Goldstone bosons, we could instead consider the scalar resonances and the pseudo-Goldstone bosons in the intermediate states. This is left for future work.

The pseudoscalar spectral function is related to the pseudoscalar form factors calculated here in a simple way

$$
\operatorname{Im} \Pi_{P^{a}}(s)=\sum_{i} \pi \delta\left(s-m_{P_{i}}^{2}\right)\left|H_{i}^{a}(s)\right|^{2}
$$

where $\delta(x)$ is the standard Dirac $\delta$-function, $m_{P_{i}}$ denotes the masses of the pseudo-Goldstone bosons and the pseudoscalar resonances with the same isospin as the considered spectral function. Finally, $H_{i}^{a}(s)$ is the corresponding pseudoscalar form factor

$$
H_{i}^{a}(s)=\frac{1}{B}\left\langle 0\left|i \bar{q} \gamma_{5} \lambda_{a} q\right| P_{i}\right\rangle .
$$

The calculation of the pseudoscalar form factor for the pseudo-Goldstone boson consists of evaluating the Feynman diagrams (a)-(d) in Fig. 2. Only the Feynman diagram (e) in Fig. 2 is relevant for the calculation of the pseudoscalar form factor for the pseudoscalar resonances. This diagram stems from the operators in the second line of Eq. (10). Moreover, we also consider the effects from the wave function renormalization of the pseudo-Goldstone bosons and express in the final results the pion decay constant $F$ in the chiral and large $N_{C}$ limits by the physical one $F_{\pi}$. The latter was calculated in Ref. [1]. This reshuffling in the expressions was also done for the scattering amplitudes in Ref. [1].

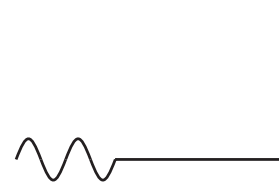

(a)

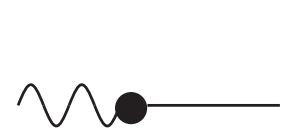

(d)

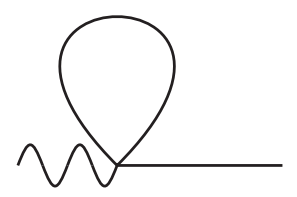

(b)

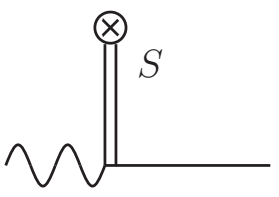

(c)

\section{$P$}

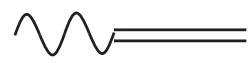

(e)

Figure 2: Pseudoscalar form factors of the pseudo-Goldstone bosons and pseudoscalar resonances. The symbols have the same meanings as those in Fig. 1, except that the wavy line stands now for the pseudoscalar external source. See the text for further details. 


\section{Remarks on semilocal duality}

In this section, we introduce the basic idea of Regge theory in order to formulate the notation used in this work. For a detailed account on Regge theory see e.g. Ref. [53]. Regge theory relates the dynamics in the $t$-channel with the $s$-channel for the scattering processes. In $\pi \pi$ scattering, the isospin well-defined amplitudes from both channels are related through [53]

$$
\begin{aligned}
& T_{\mathrm{t}}^{(0)}(s, t)=\frac{1}{3} T_{\mathrm{s}}^{(0)}(s, t)+T_{\mathrm{s}}^{(1)}(s, t)+\frac{5}{3} T_{\mathrm{s}}^{(2)}(s, t), \\
& T_{\mathrm{t}}^{(1)}(s, t)=\frac{1}{3} T_{\mathrm{s}}^{(0)}(s, t)+\frac{1}{2} T_{\mathrm{s}}^{(1)}(s, t)-\frac{5}{6} T_{\mathrm{s}}^{(2)}(s, t), \\
& T_{\mathrm{t}}^{(2)}(s, t)=\frac{1}{3} T_{\mathrm{s}}^{(0)}(s, t)-\frac{1}{2} T_{\mathrm{s}}^{(1)}(s, t)+\frac{1}{6} T_{\mathrm{s}}^{(2)}(s, t),
\end{aligned}
$$

where the subscript of $T$ denotes the $t$ - or $s$-channel and the superscript refers to the proper isospin quantum number $I$ in the $t$ - or $s$-channel, respectively.

The object of the Regge theory is the fixed- $t$ scattering amplitude and the proposed quantity to quantify the fulfillment of semilocal (or average) duality between Regge theory and hadronic degrees of freedom (h.d.f.) in Ref. [54] is

$$
\int_{\nu_{1}}^{\nu_{2}} \nu^{-n} \operatorname{Im} T_{\mathrm{t}, \text { Regge }}^{(I)}(\nu, t) d \nu=\int_{\nu_{1}}^{\nu_{2}} \nu^{-n} \operatorname{Im} T_{\mathrm{t}, \text { Hadrons }}^{(I)}(\nu, t) d \nu,
$$

where $\nu=\frac{s-u}{2}=\frac{2 s+t-4 m_{\pi}^{2}}{2}$ and $s, t, u$ are the standard Mandelstam variables. The "averaging" should take place over at least one resonance tower. Therefore, the integration region $\nu_{2}-\nu_{1}$ is typically taken as a multiple of $1 \mathrm{GeV}^{2}$. In this work, we shall focus on the energy region below $2 \mathrm{GeV}^{2}$. Semilocal duality should be well satisfied for forward scattering, i.e. with $t=0$, where the leading Regge trajectory (the one taken here into account) dominates. Like in Ref. [54], we also evaluate Eq. (36) at the threshold point $t=t_{\mathrm{th}}=4 m_{\pi}^{2}$ to show the robustness of the approach under changes of $t$ (still small compared with $\mathrm{GeV}^{2}$ ). Different choices for $n$ enable us to test the different energy regions that dominate the integrals in Eq. (36). For negative values of $n$, the dynamics in the high energy region definitely plays a more important role and it is beyond the scope of the current work. For large positive values of $n$, the integrations in Eq. (36) are then dominated by the very low energy physics, where resonances marginally contribute. In this work we test semilocal duality by taken $n$ from 0 to 3 , as suggested in Ref. [54], which is an adequate choice for the intermediate energy region from threshold up to $2 \mathrm{GeV}^{2}$, where several resonances contribute.

To evaluate the left hand side of Eq. (36), the Regge asymptotic results continued down to threshold for $\operatorname{Im} T_{\mathrm{t}, \text { Regge }}^{(I)}(\nu, t)$ at fixed $t$ are needed. The explicit formulae and detailed discussions can be found in Ref. [54] and references therein. We do not repeat here the formulation. For the right hand side of Eq. (36), one can decompose the isospin amplitudes into a sum of partial waves

$$
\operatorname{Im} T_{\mathrm{s}}^{(I)}(\nu, t)=\sum_{J}(2 J+1) \operatorname{Im} T^{I J}(s) P_{J}\left(z_{s}\right),
$$

where $z_{s}=1+2 t /\left(s-4 m_{\pi}^{2}\right)$ is the cosine of the scattering angle in the $s$-channel center of mass frame and $P_{J}\left(z_{s}\right)$ denotes the Legendre polynomials. By substituting Eq. (37) into Eq. (35) $\operatorname{Im} T_{t, \text { Hadrons }}^{(I)}(\nu, t)$ is obtained and then the right hand side of Eq. (36) can be compared with the results from Regge theory.

In order to quantify the fulfillment of semilocal duality we define, as in Ref. [54], two types of ratios of integrals like these in Eq. (36), instead of comparing directly the value of the integration from the Regge theory and the h.d.f. . The first one, $R_{n}^{I}$ is defined as:

$$
R_{n}^{I}=\frac{\int_{\nu_{1}}^{\nu_{2}} \nu^{-n} \operatorname{Im} T_{\mathrm{t}}^{(I)}(\nu, t) d \nu}{\int_{\nu_{1}}^{\nu_{3}} \nu^{-n} \operatorname{Im} T_{\mathrm{t}}^{(I)}(\nu, t) d \nu}
$$

To make closer the comparison with Ref. [54], we set in the following $\nu_{1}$ at threshold, $\nu_{2}=1 \mathrm{GeV}^{2}$ and $\nu_{3}=2 \mathrm{GeV}$. 
Other interesting objects to consider are the Finite Energy Sum Rule (FESR) between different isospin amplitudes with the same upper integration limit

$$
F_{n}^{I I^{\prime}}=\frac{\int_{\nu_{1}}^{\nu_{\max }} \nu^{-n} \operatorname{Im} T_{\mathrm{t}}^{(I)}(\nu, t) d \nu}{\int_{\nu_{1}}^{\nu_{\max }} \nu^{-n} \operatorname{Im} T_{\mathrm{t}}^{\left(I^{\prime}\right)}(\nu, t) d \nu},
$$

where $\nu_{\max }=1 \mathrm{GeV}^{2}$ or $2 \mathrm{GeV}^{2}$ in the later discussions.

Among the various cases to investigate semilocal duality between h.d.f. and Regge theory in $\pi \pi$ scattering, the golden mode is the isotensor one in the $t$-channel, since then the Regge exchange is highly suppressed (as there are no $\bar{q} q$ states with $I=2$ ). As a result, the ratios $F_{n}^{21}$ and $F_{n}^{20}$ should tend to vanish in order to satisfy semilocal duality. In contrast, the dual direct $s$-channel allows the exchanges of several resonances with isospin $I=0,1$, see Eq. (35). Thus, if semilocal duality is fulfilled, one should expect the cancellation between the different resonances exchanged in the $s$-channel, which sheds light on the resonance properties. Indeed, it establishes serious relations between the scalar and vector spectra, as we discuss later.

Finally, for the evaluation of the quantities in Eqs. (38) and (39) that quantify semilocal duality, the key ingredients are the partial waves $\operatorname{Im} T^{I J}(s)$ in Eq. (37), which were studied by us in Refs. [1, 37]. This method is different from the one used in Ref. [54], where the resonances are regenerated by unitarizing the perturbative $\chi \mathrm{PT}$ amplitudes within the IAM $[42,43]$. An important difference is that instead of the explicit resonance states, as we employ here, the IAM depends on the LECs from $S U(2)$ or $S U(3) \chi \mathrm{PT}[2,3,4]$. Nevertheless, including the explicit resonance states in the Lagrangian is not enough to guarantee that one can apply the theory to higher energies due to the important contribution from the pseudo-Goldstone boson loops. In this case, one needs to resum the unitarity chiral loops and a sophisticated approach based on the N/D method is formulated in Ref. [26], and already used to construct the unitarized scalar form factors in the previous section where we also discussed why $U(3) \chi \mathrm{PT}$ should be unitarized. Through the procedure to resum the chiral loops, in addition to extending the application energy region of $\mathrm{R} \chi \mathrm{T}$, one also generates physical resonances with finite widths $[25,26,79,80,81,22,1]$ in contrast to the zero-width resonances in the bare chiral Lagrangian. In the following, we employ the partial wave amplitudes from this procedure to analyze the semilocal duality.

\section{$5 \quad$ Phenomenological Discussions}

In this section, we study the phenomenological results of the several different types of spectral-function sum rules presented in Eq. (17) and the ratios in Eqs. (38) and (39) that quantify the fulfillment of semilocal duality. In order to perform the analyses, we need to provide the values for the resonance parameters, the low energy constants in Eqs. (11) and (15) and the subtraction constants in Eq. (27) introduced by the unitarization procedure.

We want to stress that all the parameters in the form factors already appear in the meson-meson scattering amplitudes and the pseudo-Goldstone masses. Thus, once we determine them by fitting the scattering data and the $\eta$ and $\eta^{\prime}$ masses, the form factors and the spectral functions are all predictions.

\subsection{Former Fit}

We employ here the best fit in Eq. (55) of Ref. [1], which is referred as "former fit" from now on, to calculate the form factors and spectral functions. In Figs. 3 and 4, we show in order the results for the pion scalar form factors in the quark flavor basis and the spectral functions with $a=0,3$ and 8 .

The quadratic pion scalar radius defined in Eq. (31) is found to be

$$
\left\langle r^{2}\right\rangle_{S}^{\pi}=0.43 \pm 0.01 \mathrm{fm}^{2}
$$

Compared to the dispersive result from Ref. [82]

$$
\left\langle r^{2}\right\rangle_{S}^{\pi}=0.61 \pm 0.04 \mathrm{fm}^{2}
$$

our result is around a $30 \%$ smaller than this well accepted value [82, 83]. The main reason for getting a smaller value for the pion scalar radius is that our predictions for the low energy constants $L_{4}$ and $L_{5}$ are quite small by 
using the resonance parameters from the best fit in Ref. [1]

$$
\begin{aligned}
& L_{4}(\mu=770 \mathrm{MeV})=\left(0.03_{-0.05}^{+0.08}\right) \times 10^{-3}, \\
& L_{5}(\mu=770 \mathrm{MeV})=\left(0.26_{-0.18}^{+0.11}\right) \times 10^{-3} .
\end{aligned}
$$

These two low energy constants are important in the determination of the pion scalar radius [77]. So in order to improve its determination, we need to increase the values of $L_{4}$ and $L_{5}$ from the resonance contribution. In Ref. [23], it is realized that around $50 \%$ of $L_{5}$ is in fact contributed by a second nonet of scalar resonances. Thus we decide to include a second multiplet of scalar resonances in our discussion so as to achieve a bigger pion scalar radius. This requires us to update the fit we did in Ref. [1], which we discuss in detail in the next section. Another motivation to start a new fit is that we also include the pseudoscalar resonances in the present work, which turn out to be important in the spectral-function sum rules that we discuss below.
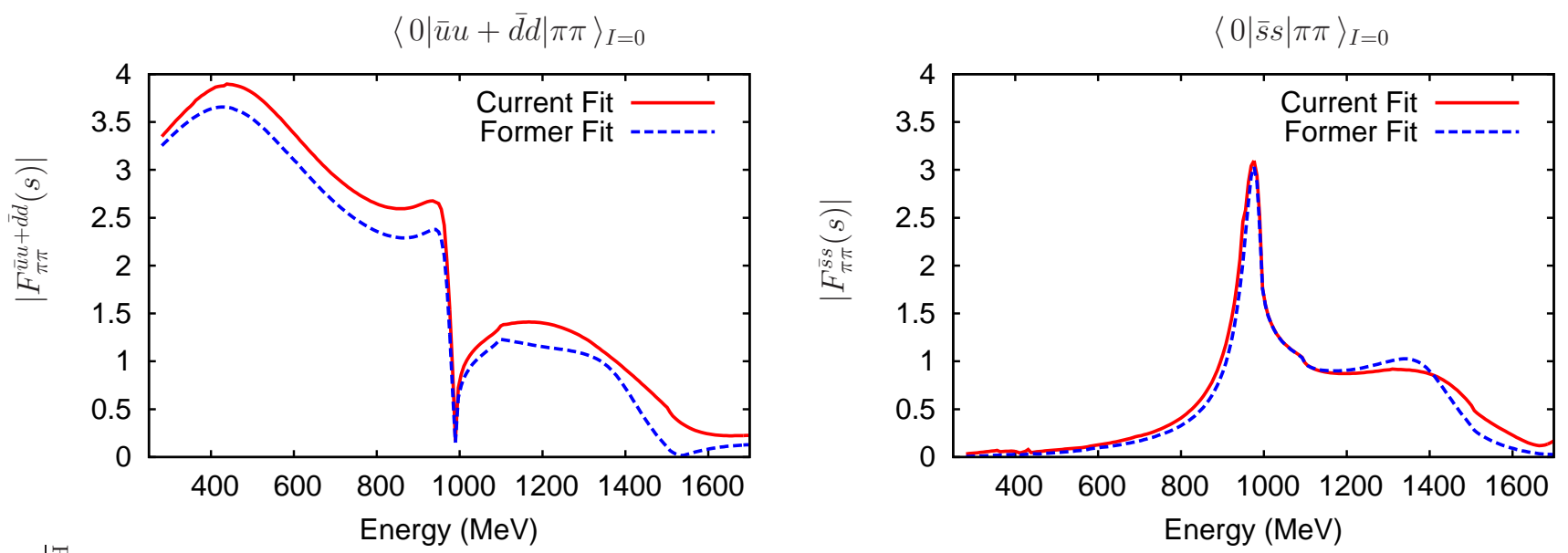

Figure 3: The $\bar{u} u+\bar{d} d$ (left panel) and $\bar{s} s$ (right panel) scalar form factors of the pion in the quark flavor basis. The (red) solid lines are for the current fit and the (blue) dashed ones for the former fit.

\section{Current Fit}

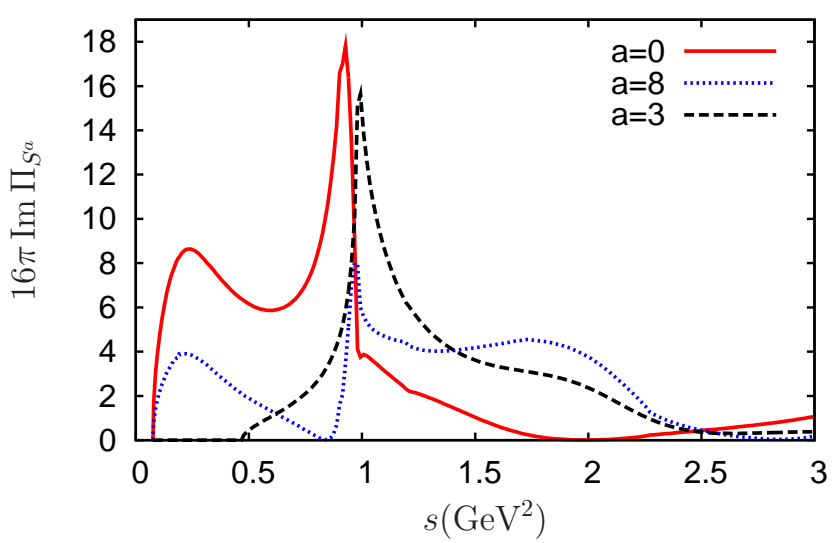

Former Fit

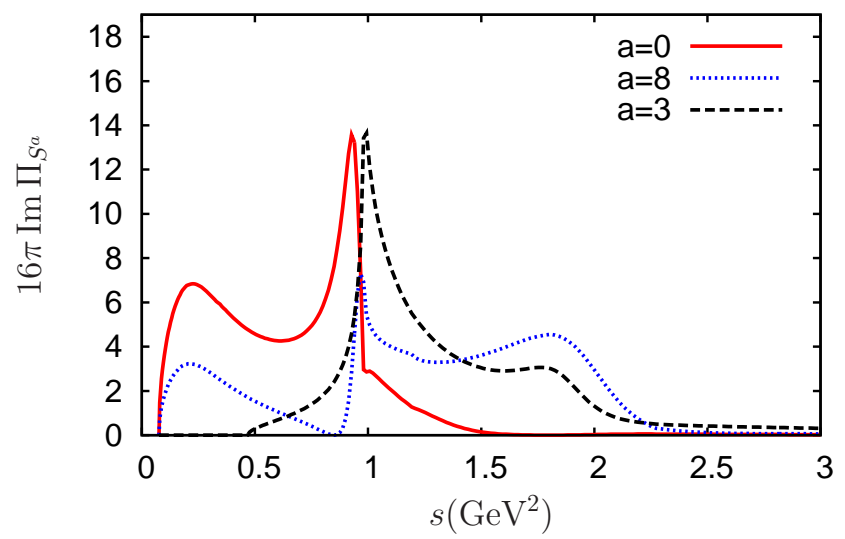

Figure 4: The scalar spectral functions for the $a=0$ (solid line), 8 (dotted line) and 3 (dashed line) correlators. The left panel is for the current fit and the right one for the former fit. 


\subsection{Current Fit}

Since we do not include the $4 \pi$ channel which turns out to be important in the energy region above $1.3 \mathrm{GeV}$ in the isoscalar scalar case [35], we fit the scattering data in the $I J=00$ channel up to $1.3 \mathrm{GeV}$ as done in Ref. [1]. In this way, the second multiplet of scalar resonances around $2 \mathrm{GeV}[22,23]$ behaves more like a background in our study and we cannot fit its parameters in a precise manner. Instead, we take the values for the resonance parameters of the second multiplet of scalar resonances from the preferred fit of Ref. [22], given in its Eq. (6.10): ${ }^{3}$

$$
\begin{aligned}
c_{d}^{\prime} & =\sqrt{3} \widetilde{c}_{d}^{\prime}=c_{m}^{\prime}=\sqrt{3} \widetilde{c}_{m}^{\prime} \simeq 40 \mathrm{MeV}, \\
M_{S_{1}^{\prime}} & =M_{S_{8}^{\prime}}=2570 \mathrm{MeV} .
\end{aligned}
$$

In this reference the scattering data in the $I J=\frac{1}{2} 0$ and $I J=\frac{3}{2} 0$ channels were investigated in a similar approach as we use here up to around $2 \mathrm{GeV}$. About the pseudoscalar resonances, we take $d_{m}=\sqrt{3} \widetilde{d}_{m}=30 \mathrm{MeV}$ and $M_{P_{8}}=M_{P_{1}}=1350 \mathrm{MeV}$, which lie in the commonly determined regions of these parameters $[41,20,17]$.

In Ref. [1] we found that the scalar resonance couplings from the fit perfectly obey the large $N_{C}$ relations

$$
\begin{aligned}
\widetilde{c}_{d} & =\frac{c_{d}}{\sqrt{3}}, \\
\widetilde{c}_{m} & =\frac{c_{m}}{\sqrt{3}},
\end{aligned}
$$

so that in the current fit we also impose them. For the subtraction constants we always keep the isospin constraints on them [1]. Finally, in the updated fit we have 18 free parameters and the fit results are

$$
\begin{array}{rlrl}
c_{d} & =\left(19.8_{-5.2}^{+2.0}\right) \mathrm{MeV} & c_{m} & =\left(41.9_{-9.2}^{+3.9}\right) \mathrm{MeV} \\
M_{S_{8}} & =\left(1397_{-61}^{+73}\right) \mathrm{MeV} & M_{S_{1}} & =\left(1100_{-63}^{+30}\right) \mathrm{MeV} \\
M_{\rho} & =\left(801.2_{-6.9}^{+8.2}\right) \mathrm{MeV} & M_{K^{*}} & =\left(910.0_{-9.1}^{+7.0}\right) \mathrm{MeV} \\
G_{V} & =\left(62.1_{-2.1}^{+1.9}\right) \mathrm{MeV} & a_{S L}^{10, \pi \eta} & =2.0_{-4.5}^{+3.3} \\
a_{S L}^{00, \pi \pi} & =-1.27_{-0.12}^{+0.12} & a_{S L}^{00, K \bar{K}} & =-0.95_{-0.16}^{+0.33} \\
a_{S}^{\frac{1}{2} 0, K \pi} & =-1.12_{-0.17}^{+0.12} & a_{S L}^{\frac{1}{2} 0, K \eta} & =-0.08_{-1.04}^{+0.38} \\
a_{S L}^{\frac{1}{2} 0, K \eta^{\prime}} & =-1.25_{-1.23}^{+1.11} & \delta L_{8} & =0.23_{-0.19}^{+0.29} \times 10^{-3} \\
M_{0} & =\left(951_{-50}^{+50}\right) \mathrm{MeV}^{-19} & \Lambda_{2} & =-0.37_{-0.19}^{+0.19} \\
\mathcal{N} & =\left(0.76_{-0.35}^{+0.36}\right) \mathrm{MeV}^{-2} & c & =1.05_{-0.33}^{+0.43}
\end{array}
$$

with $\chi^{2} /($ degrees of freedom $)=784 /(348-18) \simeq 2.38$. For the remaining subtraction constants, we impose the following relations in the fit

$$
\begin{aligned}
& a_{S L}^{00, \eta \eta}=a_{S L}^{00, \eta \eta^{\prime}}=a_{S L}^{00, \eta^{\prime} \eta^{\prime}}=a_{S L}^{00, K \bar{K}}, \\
& a_{S L}^{20, \pi \pi}=a_{S L}^{00, \pi \pi}, \\
& a_{S L}^{\frac{3}{2} 0, K \pi}=a_{S L}^{\frac{1}{2} 0, K \pi}, \\
& a_{S L}^{10, \pi \eta^{\prime}}=a_{S L}^{10, K \bar{K}}=a_{S L}^{00, K \bar{K}},
\end{aligned}
$$

and all of the subtraction constants in the vector channels (which are barely sensitive to them while they are of natural size [62]) are set equal to $a_{S L}^{00, \pi \pi}$. The parameters $\mathcal{N}$ and $c$ in Eq. (45) are introduced to describe the $\pi \eta$ distribution

$$
\frac{d N_{\pi \eta}}{d E_{\pi \eta}}=q_{\pi \eta} \mathcal{N}\left|T_{K \bar{K} \rightarrow \pi \eta}^{10}(s)+c T_{\pi \eta \rightarrow \pi \eta}^{10}(s)\right|^{2},
$$

with $q_{\pi \eta}$ the three momentum of the $\pi \eta$ system in the center of mass frame.

\footnotetext{
${ }^{3}$ The bare mass of the second nonet of scalar resonances is not either well fixed from Ref. [22]. E.g. in its fit (6.11) its bare mass is $\sim 2 \mathrm{GeV}$. The range $2-2.5 \mathrm{GeV}$ is then a realistic one for the bare mass of the second nonet of scalar resonances as follows from Ref. [22].
} 
The resulting plots from the fit in Eq. (45) are shown in Figs. 5, 6 and 7 by the solid curves, where we have also shown the best fit results from Ref. [1] by the dashed lines. The masses of $\eta$ and $\eta^{\prime}$ from the new fit read

$$
m_{\eta}=536.7_{-39.6}^{+43.3} \mathrm{MeV}, \quad m_{\eta^{\prime}}=956_{-30.4}^{+45.9} \mathrm{MeV},
$$

which are clearly improved comparing with the ones from Ref. [1]. And the leading order mixing angle of $\eta_{1}$ and $\eta_{8}$ introduced in Eq. (23) is

$$
\theta=-\left(15.1_{-2.4}^{+2.4}\right)^{\circ}
$$

Compared to the former fit from Ref. [1], three more subtraction constants, $a_{S L}^{00, K \bar{K}}, a_{S L}^{\frac{1}{2} 0, K \eta}$ and $a_{S L}^{\frac{1}{2} 0, K \eta^{\prime}}$ are set free in the current fit Eq. (45), while we reduce now the scalar resonance parameters by explicitly imposing the large $N_{C}$ relations of Eq. (44). For the subtraction constants in the isoscalar scalar channel, $a_{S L}^{00, \pi \bar{\pi}}$ and $a_{S L}^{00, K \bar{K}}$ are compatible with the former fit within error bands. For the $I J=\frac{1}{2} 0$ channel, $a_{S L}^{\frac{1}{2} 0, K \pi}$ and $a_{S L}^{\frac{1}{2} 0, K \eta^{\prime}}$ are quite similar and obey nonet symmetry approximately, while $a_{S L}^{\frac{1}{2} 0, K \eta}$ is much more different. Nevertheless, one should notice that both $a_{S L}^{\frac{1}{2} 0, K \eta}$ and $a_{S L}^{\frac{1}{2} 0, K \eta^{\prime}}$ carry especially large error bars. About the resonance parameter $c_{d}$, its value from the new fit is larger by around $30 \%$ than the one from the former fit in Ref. [1]. This new value is closer to those determined in other works $[26,22,84]$. The figure $c_{d}=19.1_{-2.1}^{+2.4} \mathrm{MeV}$ was reported in Ref. [26], $c_{d}=23.8 \mathrm{MeV}$ and $c_{d}=26 \pm 7 \mathrm{MeV}$ were given in Refs. [22, 84], respectively. For the parameter $c_{m}$, its value from the new fit also increases around $30 \%$ compared to that from the former fit in Ref. [1]. However, the error bars accompanying $c_{m}$ are now considerably smaller. This is because in the new fit we impose the large $N_{C}$ relation for the singlet couplings, Eq. (44). Concerning the bare masses, both for the resonances (scalar and vector) and the singlet $\eta_{1}$, no appreciable changes in the new fit are seen, compared with the ones from the previous one [1]. The central value of $\Lambda_{2}$ is now around a $60 \%$ of that from the best fit in Eq. (55) of Ref. [1], though both determinations are affected by large uncertainties. For the vector resonance coupling $G_{V}$, the results from the current fit and the previous one in Ref.[1] perfectly agree with each other. Concerning $\delta L_{8}$, which is introduced to compensate the uncertainties in the pseudoscalar resonance sector, it also carries large error bars.

The quality of the current fit in Eq. (45) and the best fit in Ref. [1] is quite similar, as one can see from Figs. 5, 6 and 7 . Nevertheless, the prediction for $L_{5}$ from resonance contributions using the new fit Eq. (45) is considerably increased, compared to the results in Eq. (42), due to the inclusion of the second scalar multiplet. The resulting values for $L_{4}$ and $L_{5}$ are now

$$
\begin{aligned}
& L_{4}(\mu=770 \mathrm{MeV})=\left(0.09_{-0.04}^{+0.02}\right) \times 10^{-3}, \\
& L_{5}(\mu=770 \mathrm{MeV})=\left(0.67_{-0.17}^{+0.04}\right) \times 10^{-3} .
\end{aligned}
$$

They new value for $L_{5}$ agrees well with the recent determination $L_{5}=(0.58 \pm 0.13) \times 10^{-3}$ from the latest $\mathcal{O}\left(p^{6}\right)$ $S U(3) \chi \mathrm{PT}$ fits of Ref. [85]. Concerning $L_{4}$ the latest reference cannot pin down a precise value, giving the result $L_{4}=(0.75 \pm 0.75) \times 10^{-3}$. Our determination in Eq. (50) is compatible with the latter number given its large uncertainty. Related to the larger value for $L_{5}$ in Eq. (50), the quadratic pion scalar radius from the new fit is also improved with the resulting value

$$
\left\langle r^{2}\right\rangle_{S}^{\pi}=0.49_{-0.03}^{+0.01} \mathrm{fm}^{2},
$$

increasing around a $14 \%$ compared to the value in Eq. (40) from the former fit, Eq. (55) of Ref. [1]. The resulting scalar pion form factors and spectral functions are displayed in Figs. 3 and 4 respectively, together with the results from the former fit [1].

The resulting resonance pole positions on the complex plane from the new fit Eq. (45) are collected in Table 1. We refer to Ref. [1] for the discussions on how to perform the extrapolation from the physical sheet to the unphysical Riemann sheets. Around a resonance pole $s_{R}$, corresponding to a resonance $R$, the partial wave amplitude $T_{J}^{I}(s)_{i \rightarrow j}$ tends to

$$
T_{J}^{I}(s)_{i \rightarrow j} \rightarrow-\frac{g_{R \rightarrow i} g_{R \rightarrow j}}{s-s_{R}}
$$

By calculating the residue of the resonance pole we then obtain the product of the couplings to the corresponding decay modes, $g_{R \rightarrow i} g_{R \rightarrow j}$. The pole positions for the vector resonances $\rho(770), K^{*}(892)$ and $\phi(1020)$ agree perfectly 
with those in Ref. [1]. Only slight changes are observed for the $f_{0}(500), f_{0}(980), K_{0}^{*}(800)$ and $a_{0}(980)$ resonances. While all of the excited scalar resonances, such as the $f_{0}(1370), K_{0}^{*}(1430)$ and $a_{0}(1450)$, have larger widths in the new fit Eq. (45) than in the previous one [1].
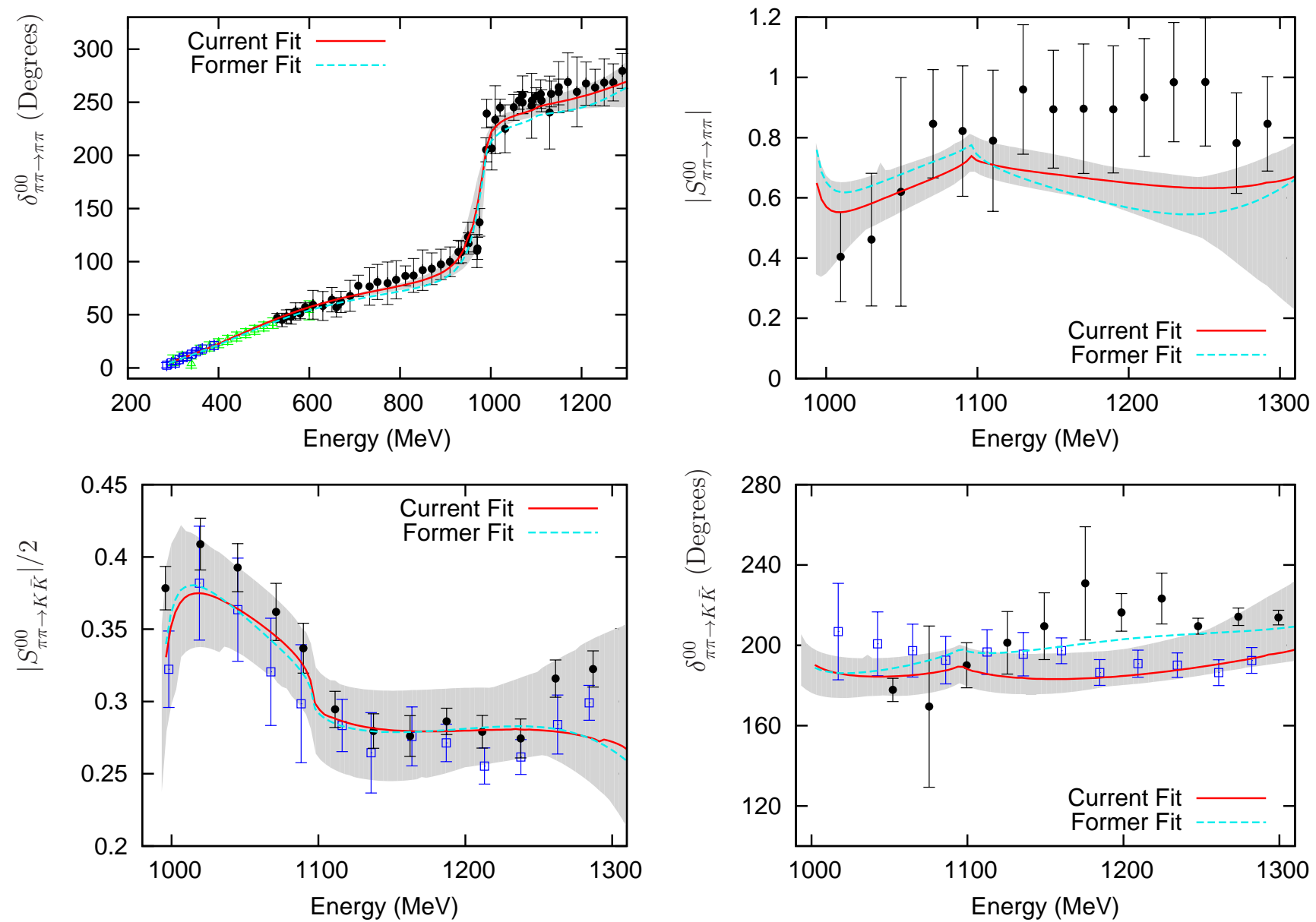

Figure 5: Plots for the $I J=00$ case from the current and former fits. From top to bottom and left to right: the $\pi \pi \rightarrow \pi \pi$ phase shifts $\left(\delta_{\pi \pi \rightarrow \pi \pi}^{00}\right)$, the modulus of the $\pi \pi \rightarrow \pi \pi$ S-matrix element $\left(\left|S_{\pi \pi \rightarrow \pi \pi}^{00}\right|\right)$, one half of the modulus of the $\pi \pi \rightarrow K \bar{K}$ S-matrix element $\left(\left|S_{\pi \pi \rightarrow K \bar{K}}^{00}\right| / 2\right)$ and the $\pi \pi \rightarrow K \bar{K}$ phase shifts $\left(\delta_{\pi \pi \rightarrow K \bar{K}}^{00}\right)$. The solid (red) line corresponds to the current fit, Eq. (45), and the dashed (blue) line represents the former fit of Ref. [1]. The error bands are represented by the shadowed areas, which are calculated using Eq. (45). The data for $\delta_{\pi \pi \rightarrow \pi \pi}^{00}$ are from Refs. [86] (green triangle), [87] (blue square) and the average data from Refs. [88, 89, 90] (black circle), as used in Ref. [26]. The data for $\left|S_{\pi \pi \rightarrow \pi \pi}^{00}\right|$ are from Ref. [88] while those for $\left|S_{\pi \pi \rightarrow K \bar{K}}^{00}\right| / 2$ are from Refs. [91] (blue square) and [92] (black circle). The phase shifts $\delta_{\pi \pi \rightarrow K \bar{K}}^{00}$ correspond to the data from Refs. [91] (blue square) and [93] (black circle). 

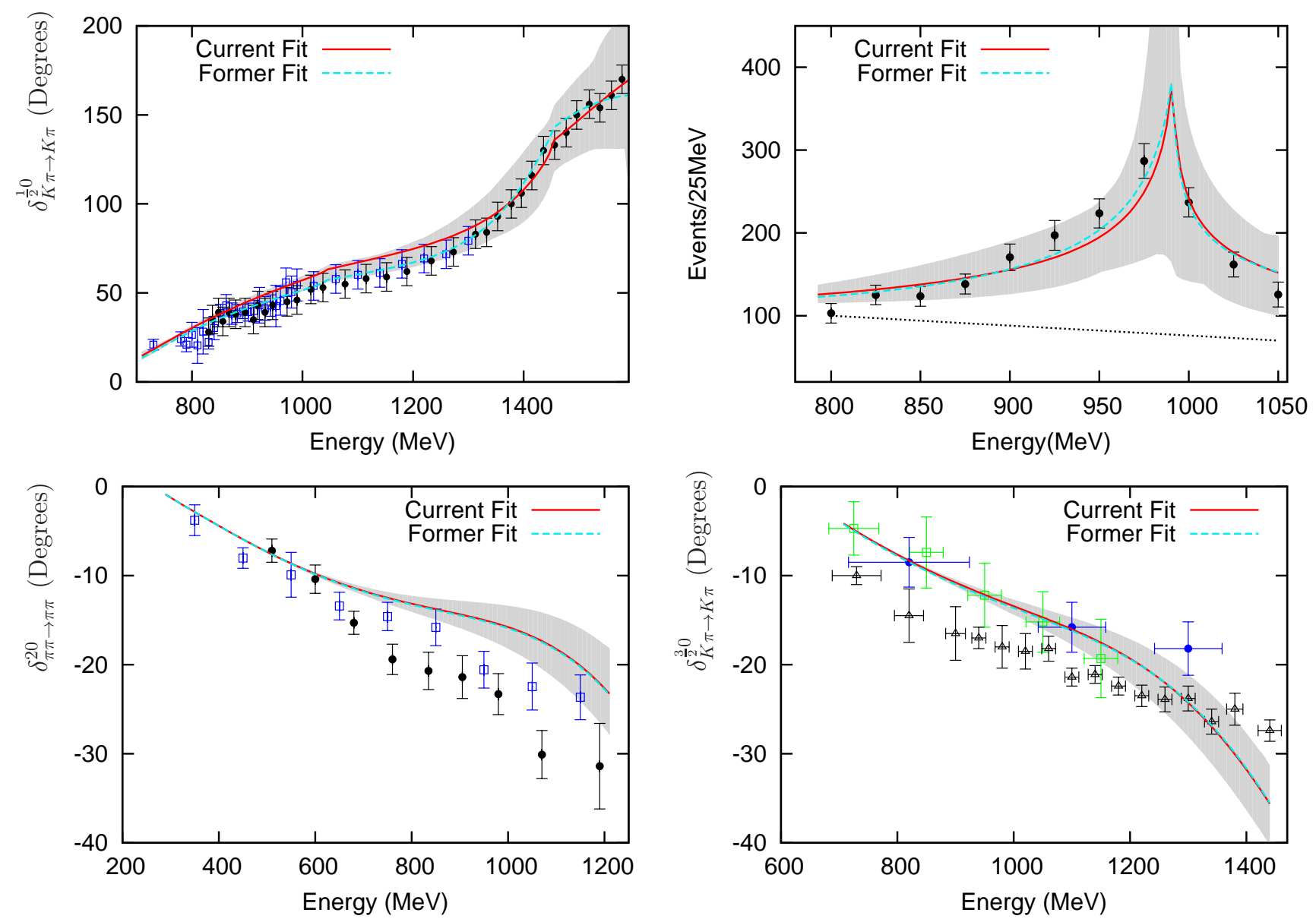

Figure 6: From top to bottom and left to right: the $K \pi \rightarrow K \pi$ phase shifts with quantum numbers $I J=\frac{1}{2} 0\left(\delta_{K \pi \rightarrow K \pi}^{\frac{1}{2} 0}\right)$, the $\pi \eta$ event distribution with $I J=10$, the $\pi \pi \rightarrow \pi \pi$ phase shifts with $I J=20\left(\delta_{\pi \pi \rightarrow \pi \pi}^{20}\right)$ and the $K \pi \rightarrow K \pi$ phase shifts with $I J=\frac{3}{2} 0\left(\delta_{K \pi \rightarrow K \pi}^{\frac{3}{2} 0}\right)$. The experimental points for $\delta_{K \pi \rightarrow K \pi}^{\frac{1}{2} 0}$ correspond to the average data from Refs. [94, 95, 96] (blue square), as employed in Ref. [26], and Ref.[97] (black circle). Data points for the $\pi \eta$ event distribution are taken from Ref. [98] and the dotted line stands for the background [26]. The data for $\delta_{\pi \pi \rightarrow \pi \pi}^{20}$ correspond to Refs. [99] (blue square) and [100] (black circle). The experimental data of $\delta_{K \pi \rightarrow K \pi}^{\frac{3}{2} 0}$ are from Refs. [101] (green square), [102] (blue circle) and [95] (black triangle). For the notation on the lines see Fig. 5 . 

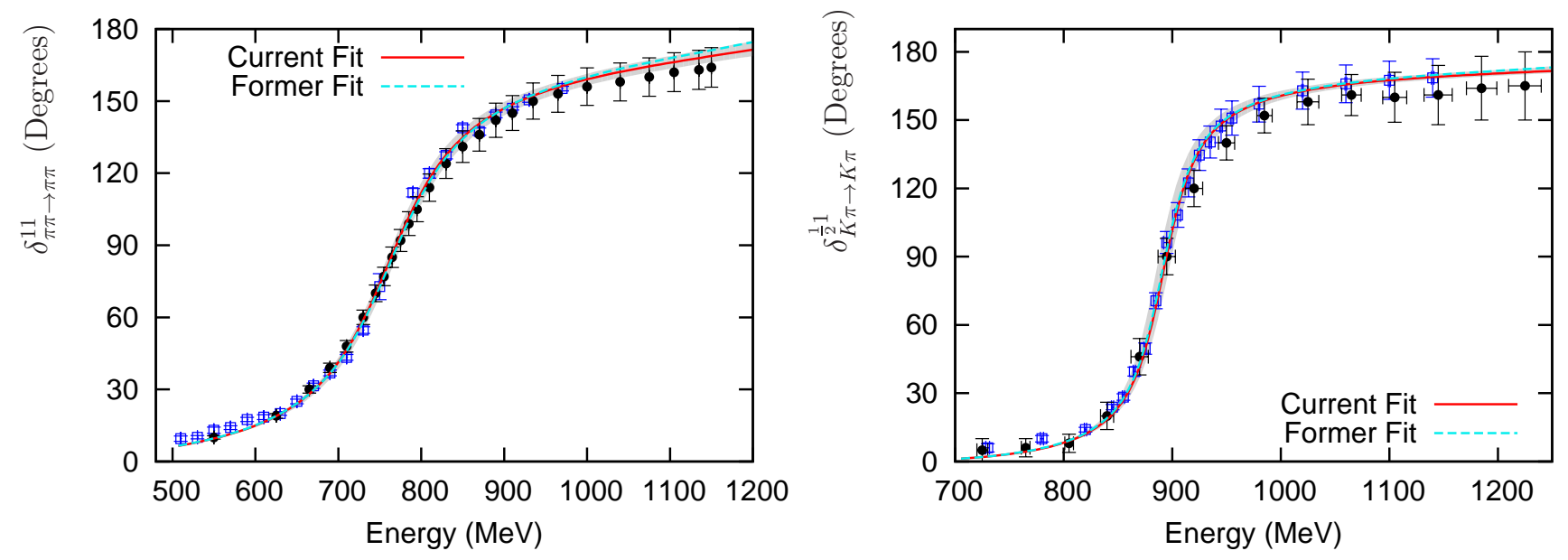

Figure 7: The left panel displays the results for the $I J=11$ channel and the right one is for the $I J=\frac{1}{2} 1$ case. The $\pi \pi \rightarrow \pi \pi$ phase shifts in the $I J=11$ case, $\delta_{\pi \pi \rightarrow \pi \pi}^{11}$, correspond to Refs. [103] (blue square) and [104] (black circle). The $K \pi \rightarrow K \pi$ phase shifts with $I J=\frac{1}{2} 1, \delta_{K \pi \rightarrow K \pi}^{\frac{1}{2} 1}$, are taken from Refs. [94] (black circle) and [95] (blue square). For the notation on the lines see Fig. 5 .

\begin{tabular}{|c|c|c|c|c|c|}
\hline $\mathrm{R}$ & $\mathrm{M}(\mathrm{MeV})$ & $\Gamma / 2(\mathrm{MeV})$ & $\mid$ Residues $^{1 / 2}(\mathrm{GeV})$ & Ratios & \\
\hline$f_{0}(500)$ & $442_{-4}^{+4}$ & $246_{-5}^{+7}$ & $3.02_{-0.04}^{+0.03}(\pi \pi)$ & $0.50_{-0.08}^{+0.04}(K \bar{K} / \pi \pi)$ & $\overline{0.17_{-0.09}^{+0.09}(\eta \eta / \pi \pi)}$ \\
\hline & & & & $0.33_{-0.10}^{+0.06}\left(\eta \eta^{\prime} / \pi \pi\right)$ & $0.11_{-0.06}^{+0.05}\left(\eta^{\prime} \eta^{\prime} / \pi \pi\right)$ \\
\hline$f_{0}(980)$ & $978_{-11}^{+17}$ & $29_{-11}^{+9}$ & $1.8_{-0.3}^{+0.2}(\pi \pi)$ & $2.6_{-0.3}^{+0.2}(K \bar{K} / \pi \pi)$ & $1.6_{-0.2}^{+0.4}(\eta \eta / \pi \pi)$ \\
\hline & & & & $1.0_{-0.2}^{+0.3}\left(\eta \eta^{\prime} / \pi \pi\right)$ & $0.7_{-0.3}^{+0.2}\left(\eta^{\prime} \eta^{\prime} / \pi \pi\right)$ \\
\hline$f_{0}(1370)$ & $1360_{-60}^{+80}$ & $170_{-55}^{+55}$ & $3.2_{-0.5}^{+0.6}(\pi \pi)$ & $1.0_{-0.3}^{+0.7}(K \bar{K} / \pi \pi)$ & $1.2_{-0.3}^{+0.7}(\eta \eta / \pi \pi)$ \\
\hline & & & & $1.5_{-0.5}^{+0.4}\left(\eta \eta^{\prime} / \pi \pi\right)$ & $0.7_{-0.3}^{+0.2}\left(\eta^{\prime} \eta^{\prime} / \pi \pi\right)$ \\
\hline$K_{0}^{*}(800)$ & $643_{-30}^{+75}$ & $303_{-75}^{+25}$ & $4.8_{-1.0}^{+0.5}(K \pi)$ & $0.9_{-0.3}^{+0.2}(K \eta / K \pi)$ & $0.7_{-0.3}^{+0.2}\left(K \eta^{\prime} / K \pi\right)$ \\
\hline$K_{0}^{*}(1430)$ & $1482_{-110}^{+55}$ & $132_{-90}^{+40}$ & $4.4_{-1.1}^{+0.2}(K \pi)$ & $0.3_{-0.3}^{+0.3}(K \eta / K \pi)$ & $1.2_{-0.2}^{+0.2}\left(K \eta^{\prime} / K \pi\right)$ \\
\hline$a_{0}(980)$ & $1007_{-10}^{+75}$ & $22_{-10}^{+90}$ & $2.4_{-0.4}^{+3.2}(\pi \eta)$ & $1.9_{-0.5}^{+0.2}(K \bar{K} / \pi \eta)$ & $0.03_{-0.03}^{+0.10}\left(\pi \eta^{\prime} / \pi \eta\right)$ \\
\hline$a_{0}(1450)$ & $1459_{-95}^{+70}$ & $174_{-100}^{+110}$ & $4.5_{-1.7}^{+0.6}(\pi \eta)$ & $0.4_{-0.2}^{+1.2}(K \bar{K} / \pi \eta)$ & $1.0_{-0.3}^{+0.8}\left(\pi \eta^{\prime} / \pi \eta\right)$ \\
\hline$\rho(770)$ & $760_{-5}^{+7}$ & $71_{-5}^{+4}$ & $2.4_{-0.1}^{+0.1}(\pi \pi)$ & $0.64_{-0.02}^{+0.01}(K \bar{K} / \pi \pi)$ & \\
\hline$\overline{K^{*}(892)}$ & $892_{-7}^{+5}$ & $25_{-2}^{+2}$ & $1.85_{-0.07}^{+0.07}(K \pi)$ & $0.91_{-0.02}^{+0.03}(K \eta / K \pi)$ & $0.41_{-0.06}^{+0.07}\left(K \eta^{\prime} / K \pi\right)$ \\
\hline$\phi(1020)$ & $1019.1_{-0.6}^{+0.5}$ & $1.9_{-0.1}^{+0.1}$ & $0.85_{-0.02}^{+0.01}(K \bar{K})$ & & \\
\hline
\end{tabular}

Table 1: Pole positions for the different resonances in $\sqrt{s} \equiv\left(\mathrm{M},-i \frac{\Gamma}{2}\right)$. The mass $(\mathrm{M})$ and the half width $(\Gamma / 2)$ are given in units of $\mathrm{MeV}$. The modulus of the square root of a residue is given in units of $\mathrm{GeV}$, which corresponds to the coupling of the resonance with the first channel (specified inside the parentheses). The last two columns are the ratios of the coupling strengths of the same resonance to the remaining channels with respect to the first one. Note that the residues for $\pi \pi, \eta \eta$ and $\eta^{\prime} \eta^{\prime}$ are given in the unitary normalization, see Ref. [1]. 


\subsection{Phenomenological results of semilocal duality}

We show in Table 2 the values of the ratios defined in Eqs. (38) and (39) from the current fit, Eq. (45). The dependence of these ratios with $N_{C}>3$ is discussed later. We also consider the contributions of the $D$-waves to the integrals in Eqs. (38) and (39), in addition to the $S$ - and $P$-waves. For including the tensor resonances, which originate the $D$-waves, we follow the formalism of Ref. [105] and take for the couplings the values determined there. The bare mass is adjusted such that the physical mass of the $f_{2}(1270)$ from the pole position agrees with the value in the PDG [36]. To avoid interrupting the current discussion, we give the expressions for the tensor contributions to meson-meson scattering in Appendix C.

In the leftmost column in Table 2 we indicate the partial waves involved in the evaluation of the integrals in Eqs. (38) and (39). The values of $n$ considered are given in the second column. In the rest of columns we give the quantities $R_{n}^{I}$ and $F_{n}^{21}$, as indicated in the top row. The values $t=0$ and $t=4 m_{\pi}^{2}$ are used in order to show the stability of the results under changes in $t$ that are small compared with $\mathrm{GeV}^{2}$. For the different quantities one should compare the numbers from Regge exchange and those obtained by including only the $(S+P)$-waves or in addition including as well the $D$-waves. Our results in Table 2 quantitatively confirm the conclusions of Ref. [54], that semilocal duality for $n=3$ with $I_{\mathrm{t}}=0$ and 1 can be perfectly satisfied by including only the $S$-and $P$-waves, while the fulfillment for $n=2$ is already marginal. For smaller values of $n$, the higher partial waves and higher cut-offs are crucial in order to satisfy semilocal duality. In this respect, we observe that once the $D$-waves are included semilocal duality is satisfied better for all the $n$ values discussed, but particularly for $n=0$ and 1 . We have also considered the role of the $\rho(1450)$ but it is negligible if one takes the $\pi \pi$ branching decay ratio from the PDG [36], which is only $6 \%$.

For $I_{\mathrm{t}}=2$ the situation is somewhat different. Before discussing the different numbers for $F_{n}^{21}$ in Table 2 , let us first comment on some specific values for the ratio $F_{n}^{21}$ in order to set up a criteria that allows one to consider a value small and then acceptable for satisfying semilocal duality. From Eq. (35), one has that $F_{n}^{21} \rightarrow-1$, if the scalar contribution is dropped (the absorptive part of the $I=2$ channel should be negligible compared with that of the scalar and vector channels). In contrast, $F_{n}^{21} \rightarrow 1$ results by neglecting the vector contribution. As we commented before, the ratio of $F_{n}^{21}$ should vanish if semilocal duality works well. Taking this in mind we then see that with the $S$ - and $P$-waves, we do not find any significant signal that semilocal duality is better satisfied for a specific value of $n$, even in some cases duality is satisfied worse for a larger value of $n$, in contrast to the situations with $I_{\mathrm{t}}=0$ and $I_{\mathrm{t}}=1$. However, in all cases the numbers are much smaller than 1 in absolute value, so that semilocal duality seems to be fulfilled quite accurately. In our scattering amplitudes higher scalar resonances are generated, instead of only the $f_{0}(500)$ as in Ref. [54], which leads to an improvement for the $I_{\mathrm{t}}=2$ channel by comparing the numbers for $F_{n}^{21}$ in Table 2 with the ones in Table VI of Ref. [54]. The masses of the heavier scalar resonances in our scattering amplitudes are close to or larger than $1 \mathrm{GeV}^{2}$, as shown in Table 1 . Hence, only their effects can be taken into account in the discussion of semilocal duality when the integration upper limit $\nu_{\text {max }}$ in Eq. (39) is larger than $1 \mathrm{GeV}^{2}$. Indeed, had we set instead $\nu_{\max }=1 \mathrm{GeV}^{2}$ the fulfillment of semilocal duality would be much worse than for the $\nu_{\max }=2 \mathrm{GeV}^{2}$ case, especially for $n=0$ and 1 . Then, in the later discussions, we only consider the ratio $F_{n}^{21}$ in Eq. (39) calculated at $\nu_{\max }=2 \mathrm{GeV}^{2}$. On the other hand, we find that the introduction of the $D$-waves, instead of narrowing the gap between the Regge prediction and the h.d.f., worsens the situation for $I_{\mathrm{t}}=2$ in the $n=0$ case. It is then advisable to focus in this work on $n>0$ for $F_{n}^{21}[106]$.

The ratios $F_{n}^{20}$ are smaller in absolute value than $F_{n}^{21}$ because the coefficient multiplying $T_{\mathrm{s}}^{(1)}$ in Eq. (35) is larger by a factor of two for $T_{\mathrm{t}}^{(0)}$ than for $T_{\mathrm{t}}^{(1)}$. In Table 2 we do not display their values since they can not reveal any new information comparing with $F_{n}^{21}$.

\subsection{Study of spectral-function sum rules}

After fixing the unknown parameters through the fit to data, we are ready to investigate the spectral-function sum rules presented in Eq. (17). To study them one has to include not only nonperturbative QCD dynamics but also perturbative QCD and operator product expansion (OPE) $[5,6]$. In this way we split the integral in two parts

$$
\int_{0}^{s_{0}}\left[\operatorname{Im} \Pi_{R}(s)-\operatorname{Im} \Pi_{R^{\prime}}(s)\right] d s+\int_{s_{0}}^{\infty}\left[\operatorname{Im} \Pi_{R}(s)-\operatorname{Im} \Pi_{R^{\prime}}(s)\right] d s=0 .
$$




\begin{tabular}{|c|c|c|c|c|c|c|c|}
\hline & $\mathrm{n}$ & $\begin{array}{l}R_{n}^{0} \\
t=t_{\mathrm{th}}\end{array}$ & $\begin{array}{l}R_{n}^{0} \\
t=0\end{array}$ & $\begin{array}{l}R_{n}^{1} \\
t=t_{\mathrm{th}}\end{array}$ & $\begin{array}{l}R_{n}^{1} \\
t=0\end{array}$ & $\begin{array}{l}F_{n}^{21} \\
t=t_{\mathrm{th}} \\
\nu_{\max }=\end{array}$ & $\begin{array}{l}F_{n}^{21} \\
t=0 \\
2 \mathrm{GeV}^{2}\end{array}$ \\
\hline \multirow[t]{4}{*}{ Regge } & 0 & 0.225 & 0.233 & 0.325 & 0.353 & $\sim 0$ & $\sim 0$ \\
\hline & 1 & 0.425 & 0.452 & 0.578 & 0.642 & $\sim 0$ & $\sim 0$ \\
\hline & 2 & 0.705 & 0.765 & 0.839 & 0.908 & $\sim 0$ & $\sim 0$ \\
\hline & 3 & 0.916 & 0.958 & 0.966 & 0.990 & $\sim 0$ & $\sim 0$ \\
\hline Ours & 0 & 0.669 & 0.628 & 0.836 & 0.817 & -0.113 & 0.040 \\
\hline$S+P$ & 1 & 0.837 & 0.812 & 0.919 & 0.908 & -0.230 & -0.087 \\
\hline \multirow[t]{2}{*}{ Waves } & 2 & 0.934 & 0.924 & 0.966 & 0.962 & -0.129 & 0.028 \\
\hline & 3 & 0.979 & 0.976 & 0.989 & 0.988 & 0.169 & 0.345 \\
\hline Ours & 0 & 0.410 & 0.400 & 0.453 & 0.468 & 0.531 & 0.587 \\
\hline$S+P+D$ & 1 & 0.653 & 0.643 & 0.694 & 0.706 & 0.154 & 0.236 \\
\hline \multirow[t]{2}{*}{ Waves } & 2 & 0.850 & 0.844 & 0.875 & 0.882 & 0.027 & 0.155 \\
\hline & 3 & 0.954 & 0.953 & 0.965 & 0.968 & 0.225 & 0.388 \\
\hline
\end{tabular}

Table 2: Current Fit: $R_{n}^{I}$ and $F_{n}^{I I^{\prime}}$ are defined in Eqs. (38) and (39), respectively. In the first column from the left the amplitudes involved in their evaluation are shown. The different values of $n$ are given in the second column. The rest of the columns correspond to $R_{n}^{I}$ and $F_{n}^{21}$ as indicated. Two values of $t, 0$ and $t_{\mathrm{th}} \equiv 4 m_{\pi}^{2}$, are considered, as shown in the second row.

The first integral, that extends along the lower-energy regime, comprises the nonperturbative region and we use our results in terms of h.d.f. to evaluate it. For the second one, higher in energy, the results from OPE are employed to evaluate the theoretical spectral functions. According to the OPE study of Ref. [10] the different spectral functions with $R=S, P$ and $R^{\prime}=S, P$ are equal in the asymptotic region in the chiral limit. ${ }^{4}$ As a result, the second integral in Eq. (53) is zero. Then, testing how well a spectral-function sum rule is satisfied reduces to evaluating the first integral, which extends along the energy region below $\sqrt{s_{0}}$. This is exactly the key object of our current study in this section.

As discussed above, we consider the strangeness conserving scalar and pseudoscalar spectral functions for $a=$ $0,8,3$. Hence there are 15 types of nontrivial spectral-function sum rules as those in Eq. (53). In order to show the results in a compact way, we display the individual values for the integration up to $s_{0}$ for each of the phenomenological spectral functions in Table 3, instead of the differences between the different spectral functions. Note that the second integral for $s>s_{0}$ is divergent, unless the difference between the spectral functions is taken as in Eq. (53). This divergent behavior is not an issue for the first integral because, as shown in Fig. 4, the phenomenological spectral functions are already very small for $s \gtrsim 2.5 \mathrm{GeV}^{2}$. In this way, the results from the integration do not depend so much on $s_{0}$ as soon as they are larger than $\sim 2.5 \mathrm{GeV}^{2}$. This vanishing behavior should be expected from Eq. (19). In the latter only a finite number of two-body channels are considered so that if the form factors vanish for $s \rightarrow \infty$ (as expected from QCD counting rules [107]) so their contribution to the spectral function does. Note also that $\rho_{i}(s)$, given in Eq. (20), tends to constant for $s \rightarrow \infty$. The definitions of the

\footnotetext{
${ }^{4}$ The calculation in Ref. [10] is done up to $\mathcal{O}\left(\alpha_{s}\right)$ and including up to dimension 5 operators.
} 
different quantities in Table 3 are

$$
\begin{aligned}
W_{i} & =16 \pi \int_{0}^{s_{0}} \operatorname{Im} \Pi_{i}(s) d s, \\
\bar{W} & =\frac{1}{3 \times 6} \sum_{i} W_{i}, \\
\sigma_{W}^{2} & =\sum_{i} \frac{\left(W_{i}-\bar{W}\right)^{2}}{17}, \quad i=S^{8}, S^{0}, S^{3}, P^{0}, P^{8}, P^{3},
\end{aligned}
$$

where we take three different values of $s_{0}$ to evaluate the integrations in order to show the dependences of the integrated results on $s_{0}$. The relative variance $\sigma_{W} / \bar{W}$ serves as a parameter to quantify how well the spectralfunction sum rules in the scalar and pseudoscalar cases hold.

Two situations by taking different masses for the pseudo-Goldstone bosons are investigated: the physical case and the chiral limit. In order to study the results at the chiral limit, it is necessary to perform the chiral extrapolation of our spectral functions. Though the resonance parameters in Eqs. (4)-(8) do not depend on the quark masses, the subtraction constants $a_{S L}$ in Eq. (27) introduced through the unitarization procedure vary with them. In Ref. [108], it is demonstrated that in the $S U(3)$ limit (as also in chiral limit) all the subtraction constants should be equal for any pseudo-Goldstone pair made of the $\pi, K$ and $\eta_{8}$ mesons. Thus we need to extrapolate the subtraction constants from the fit, which are not necessarily equal to each other, to a common value. We find that at the chiral limit such a value indeed exists in a reasonable region (roughly from -1 to 0 ), where the results of the two-point correlators are stable and the spectral-function sum rules are better satisfied compared to the physical situation. In the following, we show the typical results in this region (with a common value taken for the subtraction constant at the chiral limit of -0.5$)$.

The corresponding scalar spectral functions at the chiral limit are shown in Fig. $8 .^{5}$ It is easy to conclude from Table 3 that the spectral-function sum rules are much better fulfilled by the new fit in Eq. (45) than by the former one of [1]. The most significant changes in $W_{i}$ from both fits happen for the pseudoscalar cases, with $i=P^{0}, P^{8}$ and $P^{3}$, which are caused by the pseudoscalar resonances that are now included in the new fit. We obtain that the smallest value for the violation of the spectral-function sum rules, around $10 \%$, takes place at chiral limit by using the new fit result. Nevertheless, the result for the physical case from the new fit is also quite similar, with a violation of around $16 \%$.

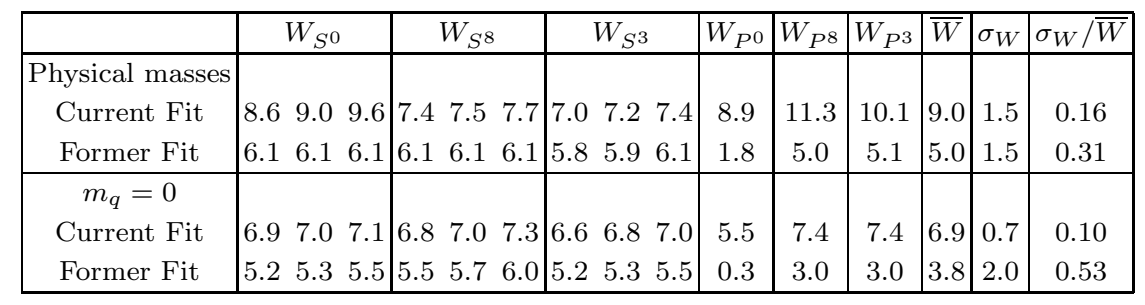

Table 3: Results from the integration of the spectral functions from 0 up to $s_{0}$, Eq. (54). We show three results in the columns $W_{S^{0}}, W_{S^{8}}$ and $W_{S^{3}}$ by taking three different values for $s_{0}: 2.5,3,3.5 \mathrm{GeV}^{2}$. The results for the pseudoscalar cases are not changed for different $s_{0}$, since the pseudoscalar spectral functions are just some Dirac $\delta$-functions Eq. (33). $W_{i}$ with $i=S^{0}, S^{8}, S^{3}, P^{0}, P^{8}, P^{3}$, the mean value $\bar{W}$ and $\sigma_{W}$ are defined in Eqs. (54)(55)(56), which are given in units of $\mathrm{GeV}^{2}$ in this table. In the last column we show the relative variance $\sigma_{W} / \bar{W}$.

\footnotetext{
${ }^{5}$ We show for later convenience the same spectral functions with $N_{C}=30$ in the right panel of Fig. 8.
} 

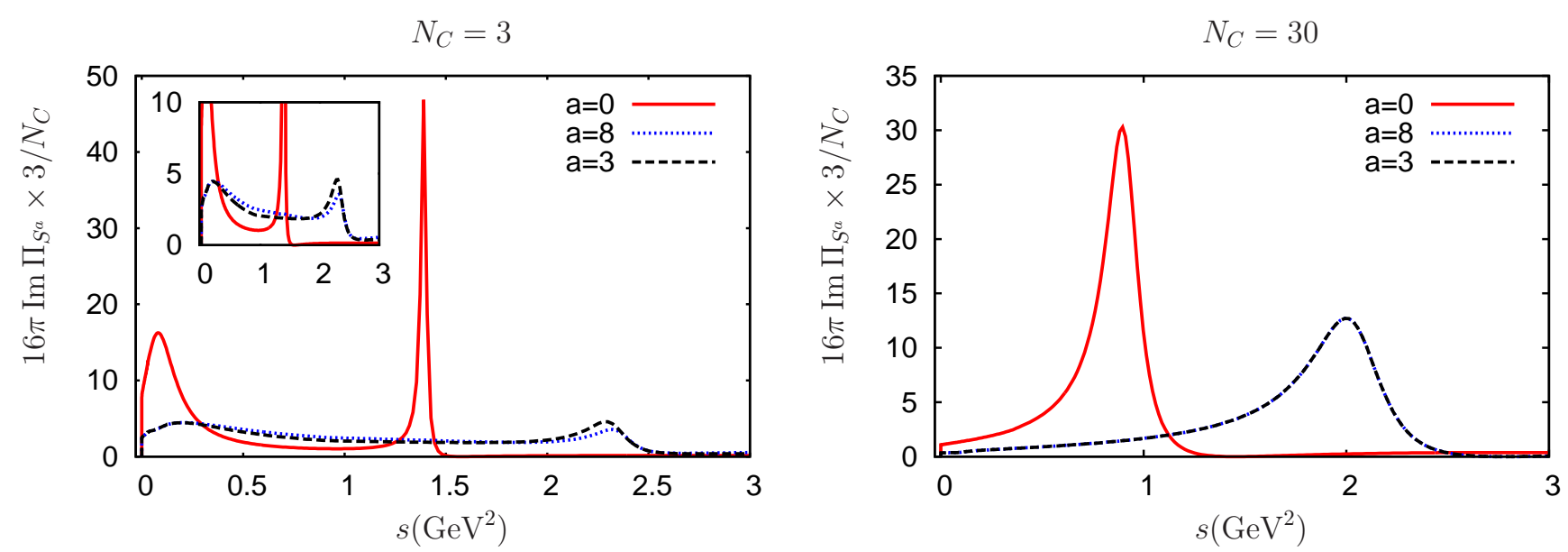

Figure 8: The scalar spectral functions at the chiral limit for $N_{C}=3$ (left panel) and $N_{C}=30$ (right panel) with the new fit of Eq. (45). The values $a=0$ (red solid line), 3 (black dashed line) and 8 (blue dotted line) are considered. The inset in the left panel shows the same figure with reducing scale, so that the spectral functions with $a=3$ and 8 are better seen.

In the left panel of of Fig. 8 the singlet spectral function (solid line) is clearly dominated in the low energy region by a peak corresponding to a pole that evolves continuously with the pseudo-Goldstone boson masses from the $f_{0}(500)$ resonance pole at the physical case. This affinity of the $f_{0}(500)$ resonance to the singlet scalar source is in agreement with the study of Ref. [109], which determined that the $f_{0}(500)$ meson was mostly a $S U(3)$ singlet. For the octet spectral function with $a=3$ one observes neatly another low energy peak (this is better seen in the inset of the left panel where the scale is changed to cover more adequately the values for $a=3$ and 8 ). This peak is due to the $a_{0}(980)$ resonance in the chiral limit, as we have checked. The higher energy peak at around $2.3 \mathrm{GeV}^{2}$ for $a=3,8$ is caused by the bare octet of scalar resonances with a common mass $M_{S_{8}} \simeq 1.4 \mathrm{GeV}$, Eq. (45), shifted to somewhat higher energies by interference with non-resonant dynamical contributions. Finally, the strong peak in the singlet spectral function at around $1.4 \mathrm{GeV}^{2}$ comes from several sources involving the bare singlet resonance $S_{1}$ with a mass $M_{S_{1}} \simeq 1.1 \mathrm{GeV}$, Eq. (45), and coupled channel dynamics with the $\eta \eta^{\prime}$ state. The lightest scalar resonances in the chiral limit were studied earlier in Ref. [26] and the pole positions for the $f_{0}(500)$ and $a_{0}(980)$ obtained there are in agreement with ours.

\section{Results from the extrapolation of $N_{C}$}

Through the fit to experimental data in Sec. 5.2, we get the unknown couplings that appear in the chiral Lagrangians as well as the subtraction constants. One advantage to employ the chiral Lagrangian approach in the phenomenological study is that once the chiral couplings are determined from one or several sets of data, we completely predict the other quantities that can be calculated from the same theory. For example, the resonance pole positions, pion scalar radius, spectral functions, spectral-function sum rules and semilocal duality that are discussed previously are all predictions from the fit to the scattering data.

Another advantage by using the chiral Lagrangian approach is to study the behavior of the various quantities by extrapolating in the number of colors of QCD, $N_{C}$, and then to confront with the results from large $N_{C}$ QCD [49]. This is straightforward in the chiral Lagrangian approach once the $N_{C}$ behavior of the parameters from the chiral Lagrangians are known $[41,4]$. Moreover, $U(3) \chi \mathrm{PT}$ is more appropriate to discuss the large $N_{C}$ running compared to $S U(3)$ or $S U(2) \chi \mathrm{PT}[3,4]$, since the singlet $\eta_{1}$, explicitly included in the $U(3)$ chiral theory, becomes the ninth pseudo-Goldstone boson in the large $N_{C}$ and chiral limits. Notice that this relevant degree of freedom is not treated as a dynamical active one in $S U(2)$ or $S U(3) \chi \mathrm{PT}[3,4]$. It is also worth stressing that the $\eta$ becomes much lighter with increasing $N_{C}$ [52], as explicitly shown in Ref. [1], an effect disregarded in previous studies $[34,42,43,44,45,46,47,48]$.

Only the leading order scaling with $N_{C}$ of the resonance parameters, such as couplings and masses, is known 
without ambiguities [41]. To show how robust is our knowledge on the $N_{C}$ behavior for the various quantities studied, the sub-leading orders for the parameters in the $N_{C}$ counting are necessary and could also be important [44, 47, 54, 1, 34]. In Ref. [54], this uncertainty induced by the sub-leading terms in the $1 / N_{C}$ expansion of the LECs is estimated approximately by taking different values of the renormalization scale $\mu$. We adopt a direct way to estimate the sub-leading order of $1 / N_{C}$ effects, that has been used in Ref. [34]. The idea is that through the fit to data, one can determine the bare resonance couplings and masses from the Lagrangian, which represent their values at $N_{C}=3$. Once their values at large $N_{C}$ are known, we perform the most general smooth extrapolation from $N_{C}=3$ to the large $N_{C}$ values up to and including $1 / N_{C}$ suppressed corrections. Of course, the values at large $N_{C}$ are not accessible directly by experiment, and can be ascertained only through theoretical considerations. In the last decades, a great progress along this line has been achieved in the analyses of short distance constraints of two- and three-point Green functions, form factors, $\tau$ decays and $\pi \pi$ scattering within $\mathrm{R} \chi \mathrm{T}[34,23,67,110,111,112,113,46]$.

The pion decay constant $F_{\pi}$ is calculated from the one-loop $U(3) \chi \mathrm{PT}$ in Ref. [1], that includes also sub-leading terms in the $1 / N_{C}$ expansion. Throughout we always consider both the leading and sub-leading $N_{C}$ scaling for $F_{\pi}$ when varying $N_{C}$ as given in Ref. [1]. The values for the fit parameters in Eq. (45) are the ones taken for $N_{C}=3$. On the other hand, due to the uncertainties of the values for the resonance parameters at large $N_{C}$, we consider several scenarios:

- Scenario 1: We take only the leading order running with large $N_{C}$ for all the resonance parameters, starting with their values at $N_{C}=3$. As discussed in more detail in Ref. [1, 41] the leading running with $N_{C}$ for the resonance parameters and meson-meson subtraction constants, $a_{S L}$, is given by:

$$
\begin{aligned}
\{ & \left.c_{d}\left(N_{C}\right), c_{m}\left(N_{C}\right), G_{V}\left(N_{C}\right), d_{m}\left(N_{C}\right)\right\} \\
= & \left\{c_{d}(3), c_{m}(3), G_{V}(3), d_{m}(3)\right\} \times \sqrt{\frac{N_{C}}{3}}, \\
\{ & M_{S_{1}}\left(N_{C}\right), M_{S_{8}}\left(N_{C}\right), M_{\rho}\left(N_{C}\right), M_{K^{*}}\left(N_{C}\right), M_{\omega}\left(N_{C}\right), M_{\phi}\left(N_{C}\right), \\
& \left.M_{P_{1}}\left(N_{C}\right), M_{P_{8}}\left(N_{C}\right), a_{S L}\left(N_{C}\right)\right\} \\
= & \left\{M_{S_{1}}(3), M_{S_{8}}(3), M_{\rho}(3), M_{K^{*}}(3), M_{\omega}(3), M_{\phi}(3),\right. \\
& \left.M_{P_{1}}(3), M_{P_{8}}(3), a_{S L}(3)\right\} .
\end{aligned}
$$

For the singlet couplings $\widetilde{c}_{d}, \widetilde{c}_{m}$ and $\widetilde{d}_{m}$, we take the large $N_{C}$ constraints in Eqs. (44) and (12).

About the $N_{C}$ running of the subtraction constant $a_{S L}$, we argue that it is natural to assume its constant behavior at large $N_{C}$, though some sub-leading $N_{C}$ corrections may exist. This is based on the fact that the unitarized scattering amplitude, defined in Eq. (28), is in fact the sum of a series of bubble diagrams with the kernel $N^{I J}(s)$, since Eq. (28) can be expanded as

$$
T^{I J}(s)=N^{I J}(s)-N^{I J}(s) g^{I J}(s) N^{I J}(s)+N^{I J}(s)\left[g^{I J}(s) N^{I J}(s)\right]^{2}+\ldots
$$

Within large $N_{C}$ QCD it is well known that the leading $N_{C}$ behavior of a meson-meson scattering amplitude, $N_{C}{ }^{\alpha}$, corresponds to $\alpha=-1$ and it can also contain other sub-leading pieces with $\alpha=-2,-3, \ldots[49]$. This feature for meson-meson scattering is inherited by the construction of $\chi \mathrm{PT}[4]$. Each single diagram in the geometric series expansion in powers of $g^{I J}(s)$ of the unitarized amplitude in Eq. (58) should decrease with $N_{C}$ at least as $1 / N_{C}$. Focusing on the first term in Eq. (58), i.e. the kernel $N^{I J}(s)$, it represents the perturbative results calculated from $\chi \mathrm{PT}$ and hence regardless of the resummation it should inherit the $N_{C}$ behavior of meson-meson scattering amplitudes dictated by large $N_{C}$ QCD. Its calculation within $\chi \mathrm{PT}$ tells us that it scales as $N_{C}^{\alpha}$ with $\alpha=-1$, including typically other sub-leading components. An immediate conclusion that follows is that the $N_{C}$ scaling index $\alpha$ for $g^{I J}(s)$ can be only an integer, following the above arguments. Moreover, $\alpha \geqslant 2$ can be also simply excluded otherwise the terms with $g^{I J}(s)$ in Eq. (58) could violate the large $N_{C}$ QCD prediction to the scattering amplitudes. The case $\alpha=0$, i.e. $g^{I J}(s)$ behaves as a constant at large $N_{C}$, is indeed the natural choice for the following reasons:

i) The relative size between a term and the next one in the expansion of Eq. (58) is $g^{I J}(s) N^{I J}(s)$. At leading order $N^{I J}(s)$ behaves as $p^{2} / F^{2}$, being $p^{2}$ a typical soft external four-momentum squared attached to 
the pseudo-Goldstone bosons. From the subtraction constant $a_{S L}$ in Eq. (27) we then have the suppression factor

$$
\frac{a_{S L} p^{2}}{(4 \pi F)^{2}} .
$$

For subtraction constants $a_{S L}$ of $\mathcal{O}(1)$ size, as the fitted values shown in Eq. (45), one then has the typical suppression for unitarity loops in $\chi \mathrm{PT}$, given in terms of the chiral symmetry breaking scale $\Lambda_{\chi P T}=4 \pi F$ [114]. In order to keep this interpretation with running $N_{C}$ it is necessary that every unitarity loop is suppressed by an extra power of $1 / N_{C}$ and, for that, the subtraction constants $a_{S L}$ should be $\mathcal{O}\left(N_{C}^{0}\right)$.

ii) The combination

$$
a_{S L}(\mu)-\log \mu^{2}
$$

in Eq. (27) is independent of the renormalization scale $\mu$. Let us consider another value $\mu^{\prime}$ for which $a_{S L}\left(\mu^{\prime}\right)=$ 0 . From the previous equation it follows that

$$
\mu^{\prime}=\mu e^{-a_{S L}(\mu) / 2}
$$

From here it is obvious that if $\left|a_{S L}(\mu)\right|$ is too different from 1 then $\mu^{\prime}$ exponentially diverges for $a_{S L}(\mu) \ll-1$ or tends to 0 for $a_{S L} \gg 1$. In both cases one has too different values from the typical one for a renormalization scale in $\chi \mathrm{PT}, \mu \sim 0.5-1 \mathrm{GeV}$, of the similar size to the previously introduced chiral symmetry breaking scale $\sim 1 \mathrm{GeV}$ or the mass of the $\rho$ resonance. The fitted values for the subtraction constants in Eq. (45) have the right size so as to keep an adequate value for $\mu^{\prime}$ which does not scale with $N_{C}$ (nor should the $a_{S L}(\mu)$ so that Eq. (61) is meaningful).

As commented in Eq. (14), since we explicitly include the resonance contributions to $L_{8}$, that grows like $N_{C}$ in the $1 / N_{C}$ expansion [41], we consider that $\delta L_{8}$ is just some remnant piece sub-leading in $N_{C}$. So we take

$$
\delta L_{8}\left(N_{C}\right)=\delta L_{8}(3)
$$

throughout the following discussion. Concerning the parameters $\Lambda_{2}$ and $M_{0}$, their leading $N_{C}$ scaling reads $[69]$

$$
\left\{\Lambda_{2}\left(N_{C}\right), M_{0}^{2}\left(N_{C}\right)\right\}=\left\{\Lambda_{2}(3), M_{0}^{2}(3)\right\} \times \frac{3}{N_{C}} .
$$

- Scenario 2: Comparing with Scenario 1, we include the sub-leading $N_{C}$ scaling for the vector resonance parameter $G_{V}$ in Eq. (5), which describes the interaction between the vector resonances and the pseudoGoldstone boson pairs, e.g. the $\rho(770) \pi \pi$ coupling. The original type of Kawarabayashi-Suzuki-RiazuddinFayyazuddin (KSRF) relation [115] predicts $G_{V}=F / \sqrt{2}$. This relation was also derived from the high energy constraint of the pion vector form factor at tree level [67]. An updated study of the vector form factor at the one-loop level [112] revealed a new version for the constraint:

$$
G_{V}=\frac{F}{\sqrt{3}} .
$$

This modified KSRF-like relation has also been confirmed in various contexts: partial wave $\pi \pi$ scattering [1, 46], radiative tau decay [113] and extra-dimension model for $\pi \pi$ scattering [116]. The large $N_{C}$ value for the pion decay constant in the chiral limit can be deduced from the $U(3) \chi \mathrm{PT}$ study of Ref. [1] with the current fit results in Eq. (45), leading to $F \simeq 80 \sqrt{\frac{N_{C}}{3}} \mathrm{MeV}$.

We impose the constraint for $G_{V}$ given in Eq. (64) at large $N_{C}$ and the extrapolation function between $N_{C}=3$ and $N_{C} \rightarrow \infty$ with $1 / N_{C}$ suppressed corrections included is:

$$
\begin{aligned}
G_{V}\left(N_{C}\right) & =G_{V}\left(N_{C}=3\right) \sqrt{\frac{N_{C}}{3}} \\
& \times\left[1+\frac{G_{V}\left(N_{C}=3\right)-G_{V}^{\mathrm{Nor}}\left(N_{C} \rightarrow \infty\right)}{G_{V}\left(N_{C}=3\right)}\left(\frac{3}{N_{C}}-1\right)\right],
\end{aligned}
$$


where $G_{V}^{\text {Nor }}\left(N_{C} \rightarrow \infty\right)=G_{V}\left(N_{C} \rightarrow \infty\right) \sqrt{\frac{3}{N_{C}}}$ and $G_{V}\left(N_{C} \rightarrow \infty\right)$ is given by Eq. (64). Notice that $G_{V}^{\text {Nor }}\left(N_{C} \rightarrow \infty\right)$ is finite in the large $N_{C}$ limit. With the running of $F_{\pi}$ as a function of $N_{C}$ from Ref. [1] we have the numerical value

$$
G_{V}^{\mathrm{Nor}}\left(N_{C} \rightarrow \infty\right) \simeq 46 \mathrm{MeV}
$$

Were the sub-leading $N_{C}$ scaling for $G_{V}$ not considered, i.e. if

$$
G_{V}^{\text {Nor }}\left(N_{C} \rightarrow \infty\right)=G_{V}\left(N_{C}=3\right)
$$

then Eq. (65) reduces to the leading behavior $G_{V}\left(N_{C}\right)=G_{V}\left(N_{C}=3\right) \sqrt{\frac{N_{C}}{3}}$, as in Eq. (57). We stress that the extrapolation function in Eq. (65) is unique if one considers only the next-to-leading order in the $1 / N_{C}$ scaling for the considered parameter. Similar extrapolation functions are also used for the other resonance parameters when needed, as specified below. For the other parameters, we keep the same setups from Scenario 1.

- Scenario 3: Here, in addition to Eq. (65) of Scenario 2, we also assume that the bare masses of the $\rho(770)$ resonance and the singlet scalar resonance $S_{1}$ (an important component of the $f_{0}(980)$ at $N_{C}=3$ ) approach to the same value at large $N_{C}$. We can realize this scenario by increasing the bare $\rho(770)$ mass by a $16 \%$ and decrease the bare $S_{1}$ mass by another $16 \%$, so that their large $N_{C}$ masses meet around $930 \mathrm{MeV}$. This value is indeed quite close to the preferred one for the $\rho(770)$ in Ref. [54] in the large $N_{C}$ limit. We take as extrapolation function the analogous one to Eq. (65):

$$
M^{2}\left(N_{C}\right)=M^{2}\left(N_{C}=3\right)\left[1+\frac{M^{2}\left(N_{C}=3\right)-M^{2}\left(N_{C} \rightarrow \infty\right)}{M^{2}\left(N_{C}=3\right)}\left(\frac{3}{N_{C}}-1\right)\right],
$$

with $M$ either the $\rho(770)$ or $S_{1}$ bare mass.

- Scenario 4: On top of the considerations in Scenario 3 we now consider the effects of the $D$-waves, which include additionally the contributions from the tensor resonances. For their resonance parameters in Eq. (C.13), we take the leading order scaling with $N_{C}$,

$$
g_{T}\left(N_{C}\right)=g_{T}(3) \times \sqrt{\frac{N_{C}}{3}}, \quad M_{T}\left(N_{C}\right)=M_{T}(3) .
$$

We summarize the different Scenarios 1-4 in Table 4. We also considered another situation in which together with the characteristics of Scenario 3 we take at large $N_{C}$ the mass of the octet of scalar resonances to be the same as that of the $S_{1}$ and $\rho(770)$. However, we checked that this new addition produces negligible contributions to the ratios $F_{n}^{I I^{\prime}}$ and $R_{n}^{I}$. The reason is because the coupling of the octet of scalar resonances to $\pi \pi$ is suppressed numerically compared with that of the singlet scalar resonance. Due to the fact that at large $N_{C}$ the $\bar{q} q$ resonances fall down to the bare mass position in the real axis, it will cause noticeable changes for the octet resonance pole trajectories. Nevertheless since the reason is obvious, we do not discuss any further this scenario.

\subsection{Semilocal duality for $N_{C}>3$}

For all the scenarios we plot in Fig. 9 the $N_{C}$ trajectories of the ratio $F_{n}^{21}$ with $t=4 m_{\pi}^{2}$, defined in Eq. (39). We verify that the results for $t=0$ are quite similar. The (red) solid line is for Scenario 1, the (green) dashed line is for Scenario 2, the (blue) dot-dashed line corresponds to Scenario 3 and the (magenta) dotted line does to Scenario 4. Between the first three scenarios the best is the third one since then the curves have the smallest absolute values for most of the $N_{C}$ axis, as required by the Regge theory and semilocal duality. The only exception is $F_{1}^{21}$ where Scenario 2 gives smaller values. Concerning Scenario 4, though it gives better results for $n=2,3$ than the others, it leads to too large values for the $n=0$ case. In Fig. 10, we show the imaginary part of the amplitudes with well defined isospin in the $t$-channel for Scenario 3 at $N_{C}=3$ and $N_{C}=30$. Their integration, according to Eq. (39), gives $F_{n}^{21}$. For $N_{C}=3$ one can clearly see in the bottom-left plot of Fig. 10 a resonant bump in the $1-3 \mathrm{GeV}^{2}$ 


\begin{tabular}{|l|l|l|l|}
\hline & $G_{V}$ & $M_{\rho}, M_{S_{1}}$ & $D$-wave \\
\hline Scenario 1 & - & - & - \\
\hline Scenario 2 & $\sqrt{ }$ & - & - \\
\hline Scenario 3 & $\sqrt{ }$ & $\sqrt{ }$ & - \\
\hline Scenario 4 & $\sqrt{ }$ & $\sqrt{ }$ & $\sqrt{ }$ \\
\hline
\end{tabular}

Table 4: Description of Scenarios 1-4. In the second and third columns the symbol - denotes that the sub-leading $N_{C}$ scaling for the corresponding parameters (indicated in the first row) is not considered. In turn, $\sqrt{ }$ denotes that the subleading $N_{C}$ scaling is taken into account. In the last column, the symbol - means that we do not consider the contribution from the $D$-waves and $\sqrt{ }$ indicates that the latter are taken into account.

region, which is absent in Ref. [54]. This is mainly contributed by the higher scalar resonance $f_{0}(1370)$, and it plays an important role to balance the contribution of the $\rho(770)$ resonance. Nonetheless, its contribution becomes less important with increasing $N_{C}$ and for $n \geq 2$ it only has a marginal contribution for all the $N_{C}$ values. When $N_{C}$ grows, the $f_{0}(500)$ resonance pole obtained in unitarized $U(3) \mathrm{R} \chi \mathrm{T}$ moves deeper and deeper in the complex energy plane and thus barely contributes. The role played in Ref. [54] by the subdominant $\bar{q} q$ component for the $f_{0}(500)$ with a mass around $1 \mathrm{GeV}$ to cancel the $\rho(770)$ contribution for $n=2,3$, is played in this work by the $f_{0}(980)$ resonance, which gradually evolves to the scalar singlet $S_{1}$ resonance and starts behaving as a $\overline{q q}$ state for $N_{C}>6$. In both works, a $\bar{q} q$ scalar state with a mass around $1 \mathrm{GeV}$ is needed in order to satisfy local duality. The evolution of the resonance poles with increasing $N_{C}$ will be discussed in detail in the next section.

Focusing on the solid (red) lines in the four panels of Fig. 9, resulting from Scenario 1, one can immediately conclude that semilocal duality, though satisfied at $N_{C}=3$ for all the values of $n$, it is not well satisfied at large $N_{C}$ for $n \geq 1$. Indeed, the situation taking place for Scenario 1 is quite similar to the one-loop IAM case of Ref. [54] where the $f_{0}(500)$ does not show a sub-leading $\bar{q} q$ component. For $n=0$, both the one- and twoloop IAM results satisfy approximately semilocal duality at large $N_{C}$, while only the results which include the $\bar{q} q$ sub-leading component for the $f_{0}(500)$ [54], show a clear sign of duality for $n=1,2$ and 3 . The appearance of this $\bar{q} q$ subdominant component, which approaches to the real axis in the complex energy plane at large $N_{C}$, plays the fundamental role in the fulfillment of duality in Ref. [54]. However, one should also keep in mind that the $N_{C}$-behavior for the $\rho(770)$ resonance in the two-loop result, with its pole position at $\sqrt{s_{\rho}}=M_{\rho}-i \Gamma_{\rho} / 2=$ $(950.0-i 34.8) \mathrm{MeV}$ for $N_{C}=12$, is quantitatively different compared with the one-loop result for the same value of $N_{C}, \sqrt{s_{\rho}}=(710.5-i 18.4) \mathrm{MeV}$ [54]. Due to the small ratios of $\Gamma_{\rho} / M_{\rho}$ for $N_{C}=12$ (in both cases it is less than 0.1 ), a reasonable approximation consists of using the narrow resonance approximate formula to estimate the $\rho(770)$ contribution in both cases to $\operatorname{Im} T^{I J=11}(s)$. It reads [53]

$$
\operatorname{Im} T^{11}(s)=16 \pi^{2} \frac{M_{\rho} \Gamma_{\rho}}{\bar{\sigma}\left(M_{\rho}^{2}\right)} \delta\left(s-M_{\rho}^{2}\right)
$$

where $\bar{\sigma}(s)=\sqrt{1-4 m_{\pi}^{2} / s}$. We have adjusted the normalization of the partial wave amplitude in Eq. (70) to the one defined in Ref. [1], and used in this work. Now, one can analytically calculate the $\rho(770)$ contribution to the right hand side of Eq. (36), which finally enters in the ratios of Eqs. (38) and (39). By applying Eq. (70), the $\rho(770)$ contribution to the FESR for $I_{\mathrm{t}}=2$ in terms of its width and mass is

$$
\int_{\nu_{1}}^{\nu_{\max }} \nu^{-n} \operatorname{Im} T_{t, \rho}^{I=2}(\nu, t) d \nu=-24 \pi^{2} \frac{\Gamma_{\rho} M_{\rho}^{1-2 n}}{\bar{\sigma}\left(M_{\rho}^{2}\right)} .
$$

where the integration region between $\nu_{1}$ and $\nu_{\max }$ always covers the $\rho(770)$ mass. For simplicity we show in the previous equation the result at $t=0$ and similar results can be straightforwardly deduced for other values of $t$. The ratio of the $\rho(770)$ contribution between the two- and one-loop cases from [54] as follows from Eq. (71) is

$$
\frac{\Gamma_{\rho, \text { two-loop }}}{\Gamma_{\rho, \text { one-loop }}}\left(\frac{M_{\rho, \text { two-loop }}}{M_{\rho, \text { one-loop }}}\right)^{1-2 n} \frac{\bar{\sigma}\left(M_{\rho, \text { one-loop }}^{2}\right)}{\bar{\sigma}\left(M_{\rho, \text { two-loop }}^{2}\right)} .
$$

Taking into account the $\rho(770)$ pole positions for $N_{C}=12$ from Ref. [54], that were explicitly shown above, the ratio in the previous equation is 0.43 for $n=3$. This implies that the $\rho(770)$ contribution is reduced by more than 
$\mathrm{n}=0$

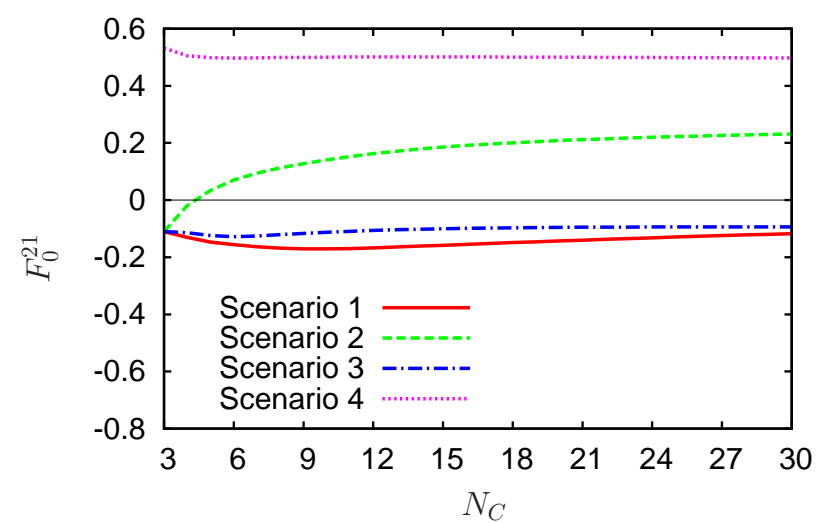

$\mathrm{n}=2$

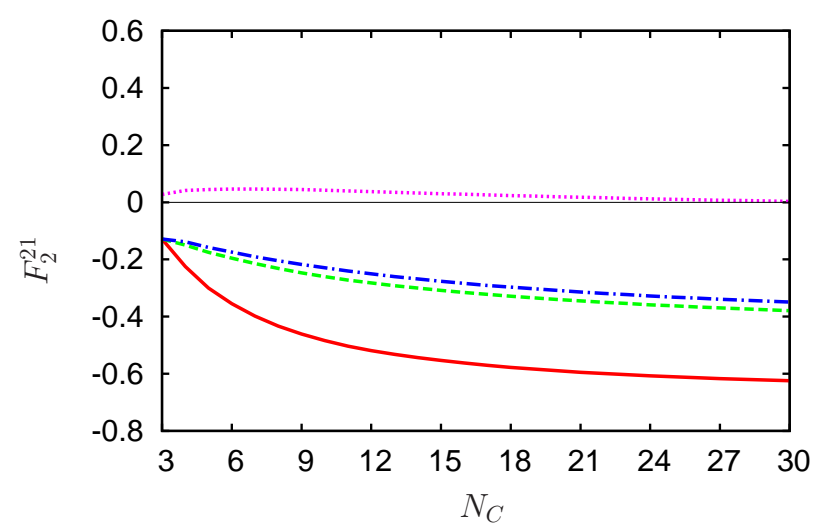

$\mathrm{n}=1$

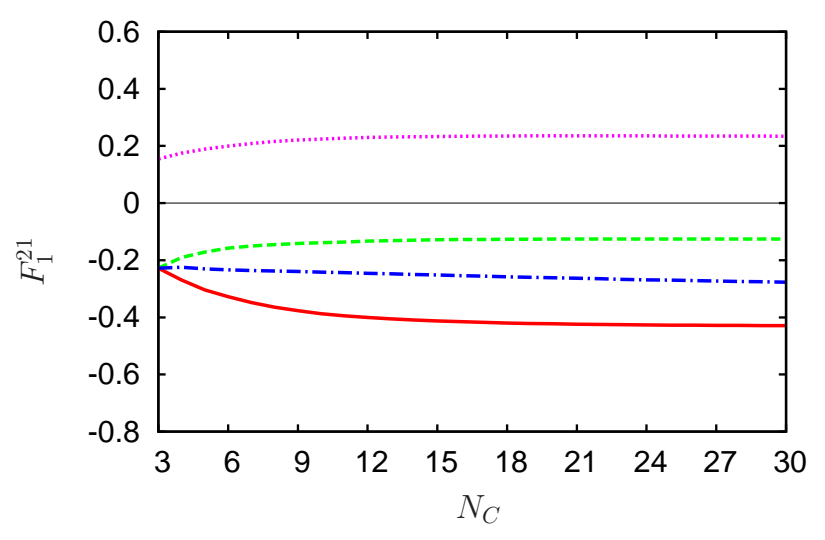

$\mathrm{n}=3$

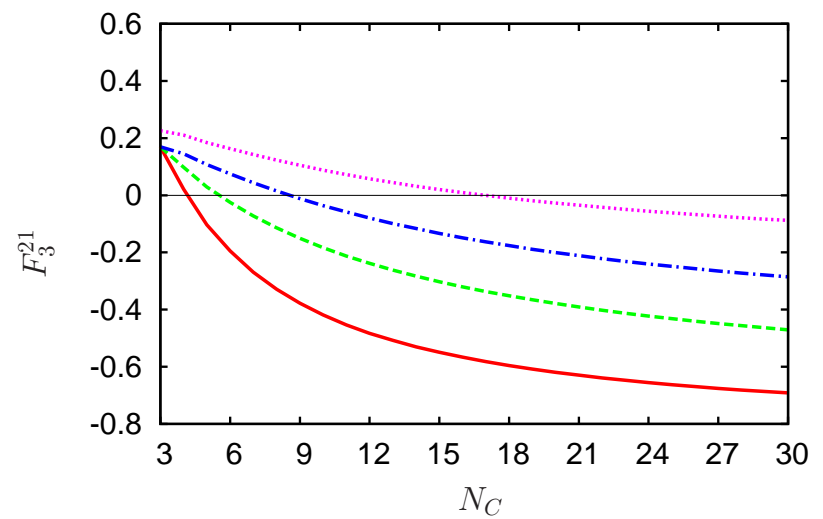

Figure 9: $F_{n}^{21}\left(t=4 m_{\pi}^{2}\right)$. The solid (red) lines correspond to the current fit and only the leading order of the $N_{C}$ scaling for the resonance parameters is considered, i.e. Scenario 1. The dashed (green) lines additionally include the sub-leading $N_{C}$ scaling for $G_{V}$, i.e. Scenario 2. The dot-dashed (blue) lines correspond to take into account the sub-leading $N_{C}$ scaling for $G_{V}, M_{\rho}$ and $M_{S_{1}}$, i.e. Scenario 3. The doted (magenta) lines Scenario 4 so that the $D$-wave contribution is included as well.

$50 \%$ in the two-loop result compared with the one-loop case in the IAM study of Ref. [54]. When increasing $N_{C}$ this ratio will stay put since the $\rho(770)$ resonance already starts to behave as a standard $\bar{q} q$ resonance at $N_{C}=12$, with its mass approaching to a constant and its width decreasing as $1 / N_{C}[42,43,54]$. Though this $N_{C}$ behavior of the $\rho(770)$ pole is not essential in Ref. [54] to satisfy local duality, it definitely helps to improve it.

This reduction of the $\rho$ signal with increasing $N_{C}$ is explained by our present approach in a quite transparent way. For that one needs to take into account the sub-leading $N_{C}$ scaling of the resonance parameters. It is also the case that when the latter are taken into account semilocal duality is also better fulfilled. Among the estimates of the sub-leading scaling for various resonance parameters, $G_{V}$ is the most reliable one, since it can be directly derived by requiring a proper high energy behavior of the partial wave amplitudes, which are the key input in the study of semilocal duality. In addition, this constraint has also been confirmed in different processes, as already discussed above $[112,1,46,113,116]$. It turns out that the large $N_{C}$ condition Eq. (64) considerably improves the fulfillment of semilocal duality. Thus, we provide another hint to confirm this constraint. This improvement is displayed in Fig. 9 by the difference between the solid (red) lines and the dashed (green) lines. The slight readjustment of the bare masses for the $\rho(770)$ and $S_{1}$ at large $N_{C}$ seems to improve the situations for $n=0$ and $n=3$, which can be seen from the differences between the dashed (green) lines and the dot-dashed (blue) ones. It causes significant effects for $n=0$ by alternating the sign of the ratio, nevertheless the magnitudes are always less than 0.2 .

Finally, we comment on the $D$-wave effects. Though the $D$-wave amplitudes can gain contributions from many sources, such as chiral loops, scalar, pseudoscalar and vector resonances exchanged in crossed channels, one expects 

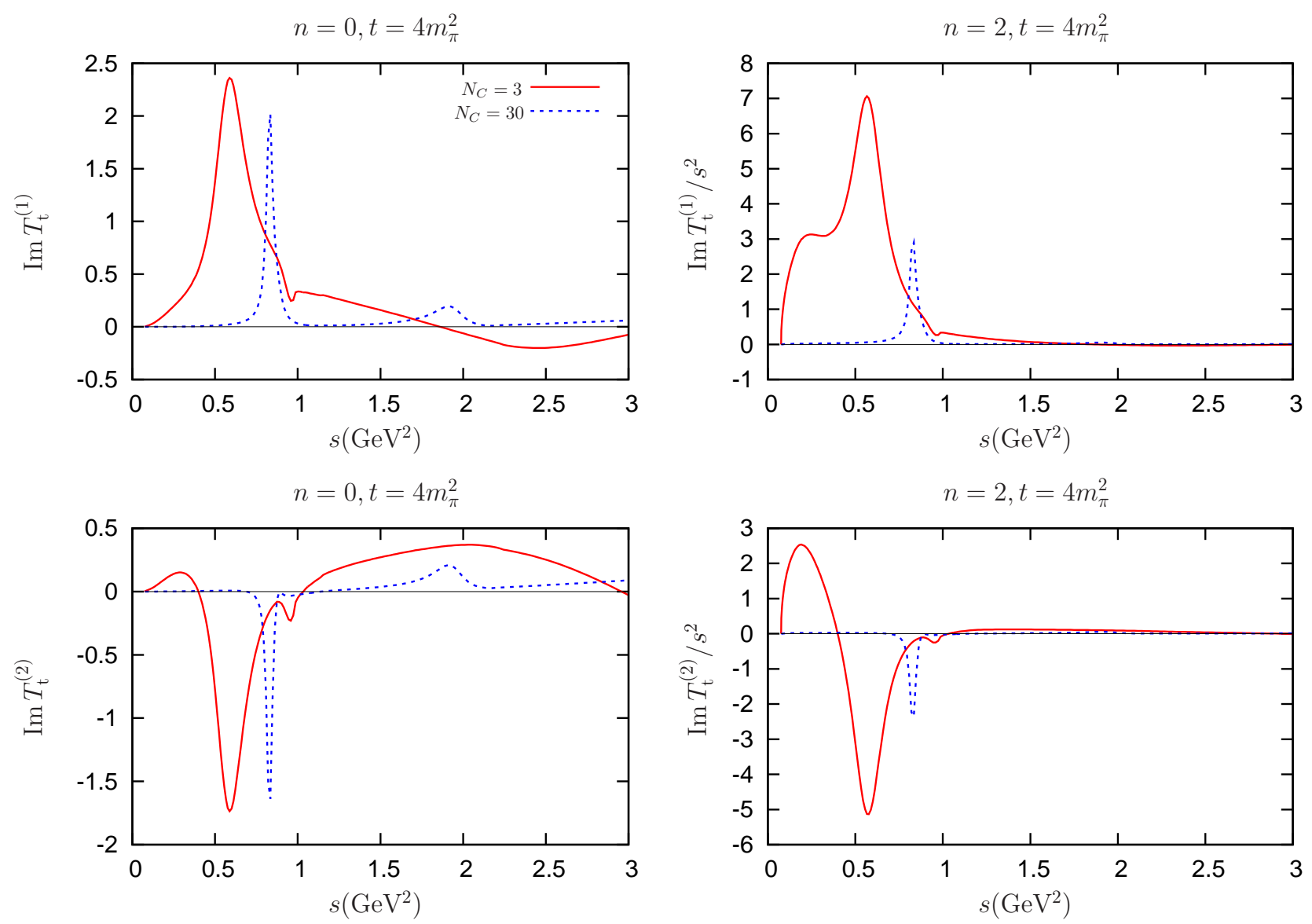

Figure 10: For Scenario 3, we show $\operatorname{Im} T_{\mathrm{t}}^{(1)}\left(t=4 m_{\pi}^{2}\right)$ in the two panels of the top row and $\operatorname{Im} T_{\mathrm{t}}^{(2)}\left(t=4 m_{\pi}^{2}\right)$ in the ones at the bottom. The results from $N_{C}=3$ (red solid lines) and 30 (dashed blue lines) are displayed for all the four figures.

from phenomenological reasons that the most important ones correspond to the tensor resonances [117, 118]. From the change between the dot-dashed (blue) lines and the dotted (magenta) ones in Fig. 9, one can discern the role that the $D$-waves play in the FESR. As one can see, it is significant for $n=0,1,2$ and slight for $n=3$ (and it should be even smaller for larger values of $n$ since then low-energy physics is enhanced, as already commented). For $n=1$, though the tensor resonances alternate the sign of the ratios, the magnitudes are still quite small when varying $N_{C}$. It also clearly improves the condition for $n=2$ and $n=3$, though for $n=0$ it clearly deteriorates the fulfillment of semilocal duality. We observe that the $D$-waves give rise to a large contribution that overbalances the one of the $\rho(770)$. This hints that higher vector resonances are necessary to cancel the $D$-wave contributions. In order to give a rough idea on whether the $\rho(1450)$ resonance in PDG [36] can counteract the $D$-wave contribution, we include another heavier vector resonance in $\pi \pi$ scattering following the formula in Eq. (70). We refer this resonance as $\rho^{\prime}$ in the following. The only difference now is that $\Gamma_{\rho^{\prime}}$ in Eq. (70) should be understood as the partial decay width $\Gamma_{\rho^{\prime} \rightarrow \pi \pi}$. Moreover, since we focus on semilocal duality below $2 \mathrm{GeV}^{2}$, we simply set the $\rho^{\prime}$ mass as $1350 \mathrm{MeV}$ in order to cover its peak for the integral in Eq. (36). We find the $\rho(1450)$-type resonance gives a negligible contribution for all the values of $n$, because of its too small decay branching ratio to $\pi \pi$ (around a $6 \%$ ), as follows from the present information in the PDG [36]. We verify that the decay branching ratio to $\pi \pi$ of the hypothetical resonance $\rho^{\prime}$ needs to be around $40 \%$ in order to decrease the large contribution by the $D$-wave for the $n=0$ case down to 0.2 . This is a hint in favor for the existence of a heavy $\rho^{\prime}$ with a significant branching decay ratio to $\pi \pi$. For $n=1,2,3$ no cancellations or just small ones are in fact needed in order to have a rather suppressed FESR with $I=2$ in the $t$ channel. Nevertheless, if included for these values of $n$ the resulting curves are equally satisfactory (even better for $n=1$ ). 


\subsection{Resonance pole trajectories with varying $N_{C}$}

Unless the opposite is stated, all the $N_{C}$ pole-trajectory evolutions studied from now on correspond to the Scenario 3 in Table 4. This is also the scenario that satisfies best semilocal duality, as seen in Sec. 6.1.

Due to the $N_{C}$ behavior of the singlet $\eta_{1}$ mass $M_{0}$ in Eq. (63), a novel feature of $U(3) \chi \mathrm{PT}$, as compared with the $S U(3)$ version, is that the masses of the pseudo-Goldstone bosons at large $N_{C}$, especially for $\eta$ and $\eta^{\prime}$, can be significantly different from their physical values at $N_{C}=3$, as shown in Ref. [1]. On the other hand, the masses of the pion and kaon barely change when varying $N_{C}$. Similar results are obtained from the new fit in Eq. (45), that are depicted in Fig. 11. One can see there that for $N_{C}=30$, the $\eta$ and $\eta^{\prime}$ masses decrease from their physical values down to around $300 \mathrm{MeV}$ and $700 \mathrm{MeV}$, respectively. We point out the $\eta^{\prime}$ retains a somewhat heavy mass at large values of $N_{C}$ mainly caused by the kaon mass [1]. This is easily seen by the leading order expression of the $\eta$ and $\eta^{\prime}$ masses from $U(3) \chi \mathrm{PT}[1]$. They result from the tree level calculation using the leading order Lagrangian Eq. (1) at the large $N_{C}$ limit, i.e. $M_{0} \rightarrow 0$ :

$$
\begin{aligned}
\bar{m}_{\eta}^{2} & =\bar{m}_{\pi}^{2}, \\
\bar{m}_{\eta^{\prime}}^{2} & =2 \bar{m}_{K}^{2}-\bar{m}_{\pi}^{2},
\end{aligned}
$$

where $\bar{m}_{\eta}$ and $\bar{m}_{\eta^{\prime}}$ stand for the $\eta$ and $\eta^{\prime}$ masses, respectively.

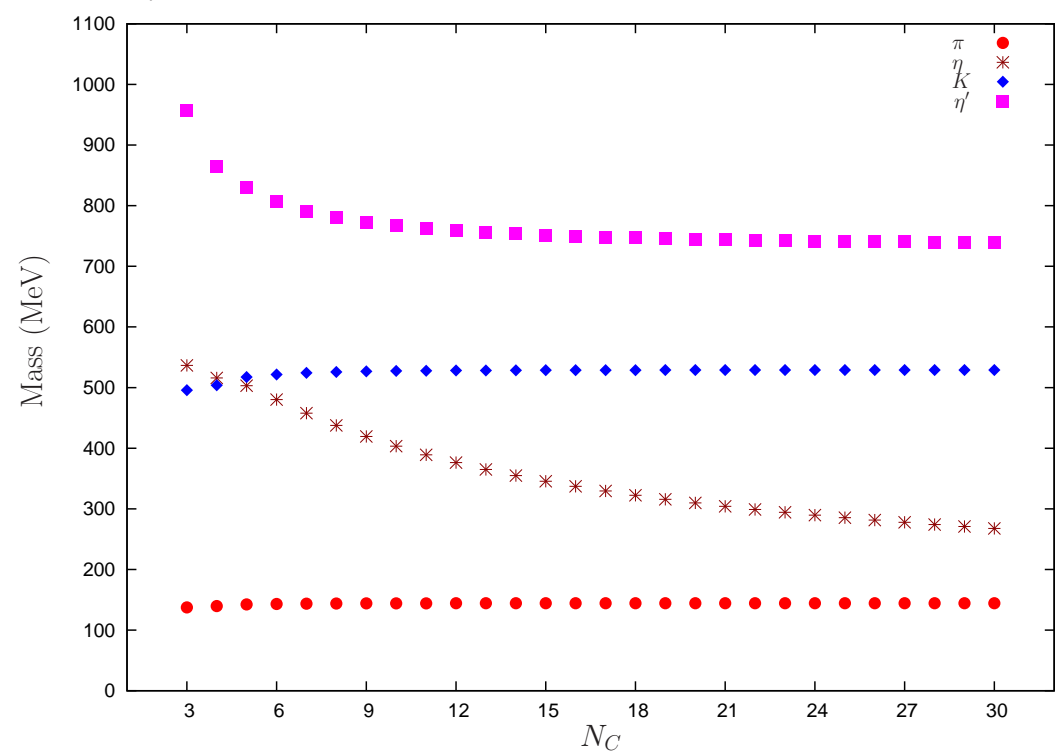

Figure 11: $N_{C}$ running for the masses of pseudo-Goldstone bosons.

The expressions that relate $\bar{m}_{\pi}^{2}$ and $\bar{m}_{K}^{2}$ with $m_{\pi}^{2}$ and $m_{K}^{2}$ at the one-loop level are given by Eqs. (A1), (B2), (B4) and (B7) of Ref. [1] to which one must add the new contribution Eq. (A.1), due to $\delta L_{8}$ and the exchange of the pseudoscalar resonances. The numerical values from the new fit in Eq. (45) for the pion and kaon leading-order masses extracted at the one-loop level are

$$
\bar{m}_{\pi}=136.4_{-1.7}^{+2.3} \mathrm{MeV}, \quad \bar{m}_{K}=499.6_{-30.4}^{+30.6} \mathrm{MeV},
$$

which leads to

$$
\bar{m}_{\eta}=136.4_{-1.7}^{+2.3} \mathrm{MeV}, \quad \bar{m}_{\eta^{\prime}}=693.3_{-43.8}^{+43.7} \mathrm{MeV}
$$

according to Eq. (73). We point out that at the chiral and large $N_{C}$ limits, all the masses of the nonet of Goldstone bosons vanish. The $N_{C}$ evolution of the leading order mixing angle in Eq. (23), given explicitly in Eq. (B7) of Ref.[1], is displayed in Fig. 12. At large $N_{C}$ it corresponds to ideal mixing, as it should. 


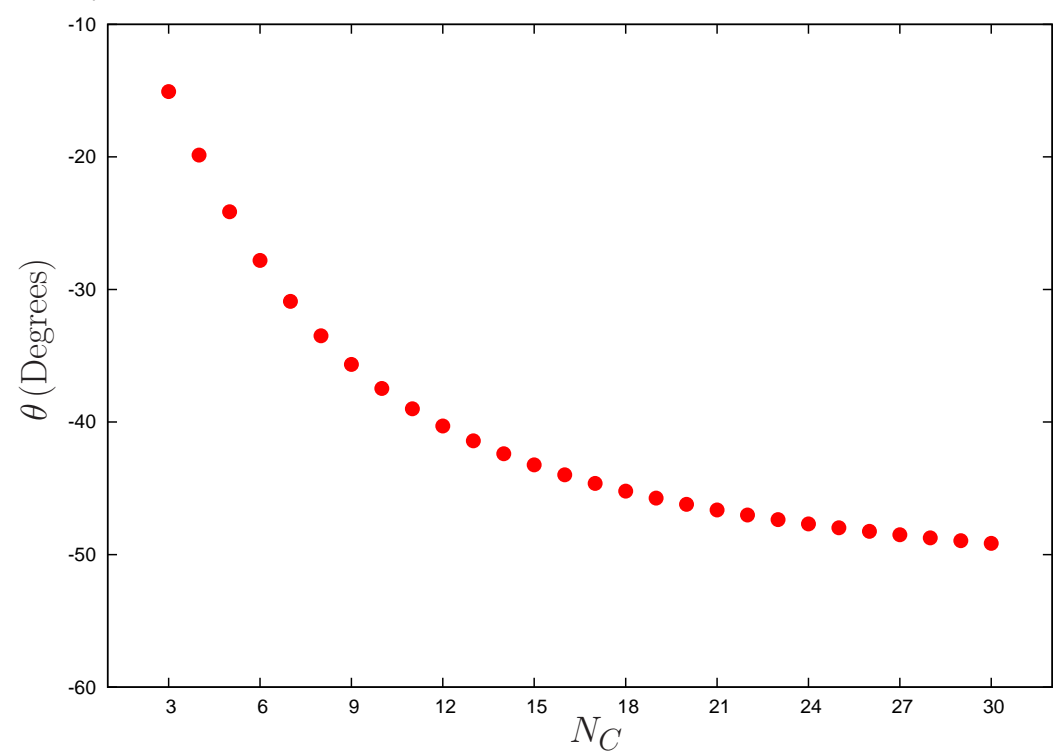

Figure 12: $N_{C}$ running for the leading order $\eta-\eta^{\prime}$ mixing angle $\theta$ introduced in Eq. (23).

In the study of the evolutions of the resonance pole positions we always take the $N_{C}$ running masses for the pseudo-Goldstone bosons as shown in Fig. 11. The resulting $N_{C}$ trajectories of the various resonance poles and their residues were studied with detail in the Ref. [1]. Qualitatively speaking we do not find any significant changes in the trajectories by using the new fit in Eq. (45) and thus we confirm the conclusions obtained in this reference: the $f_{0}(500), K_{0}^{*}(800)$ and $a_{0}(980)$ resonances go deeper in the complex energy plane when increasing the values of $N_{C}$. For the other resonances, $f_{0}(980), f_{0}(1370), K_{0}^{*}(1430), a_{0}(1450), \rho(980), K^{*}(892)$ and $\phi(1020)$, approach to the real axis and behave like the standard $\bar{q} q$ resonances at large $N_{C}$. Notice that the $f_{0}(980)$ for not so large values of $N_{C}$ does not follow the standard $\bar{q} q$ pattern (compare with the pole trajectories for the vector resonances in Fig. 15). E.g. its width indeed clearly increases up to $N_{C} \simeq 7$. This is a signal of the fact that the $f_{0}(980)$ has also a strong contribution to its nature as a $K \bar{K}$ bound state [25].

Two variant approximations, named as vector reduced and mimic $S U(3)$, were studied to explore the $N_{C}$ trajectories of the resonance pole positions in Ref. [1]. In the vector reduced case, we freeze out the full propagators of the vector resonances in the scattering amplitudes and only keep the leading local terms generated from them, which are $\mathcal{O}\left(p^{4}\right)$ in the chiral counting (or $\mathcal{O}\left(\delta^{3}\right)$ ). The purpose for introducing this approximation is to highlight the difference between using the tree level resonance exchanges and the local LECs in the meson-meson scattering amplitudes. As discussed in Ref. [1], we find that freezing the bare scalar resonances in the amplitudes does not lead to significant effects for the $f_{0}(500)$ resonance. This is why we only discuss the vector reduced case. In the approximation of mimic $S U(3)$, instead of taking the $N_{C}$ running masses for the pseudo-Goldstone bosons, we freeze them throughout and, in addition, we do not consider any $\eta-\eta^{\prime}$ mixing terms. Thus, in this case, the $\eta$ and $\eta^{\prime}$ correspond to the octet $\eta_{8}$ and singlet $\eta_{1}$ respectively. The masses of $\pi, K$ and $\eta$ are fixed at the physical values and the $\eta^{\prime}$ mass is taken from the leading order prediction of Eq. (1), which is around $1040 \mathrm{MeV}$. This mimics the conditions of $S U(3) \chi \mathrm{PT}$ that we consider for comparison with our $U(3) \chi \mathrm{PT}$ results. 

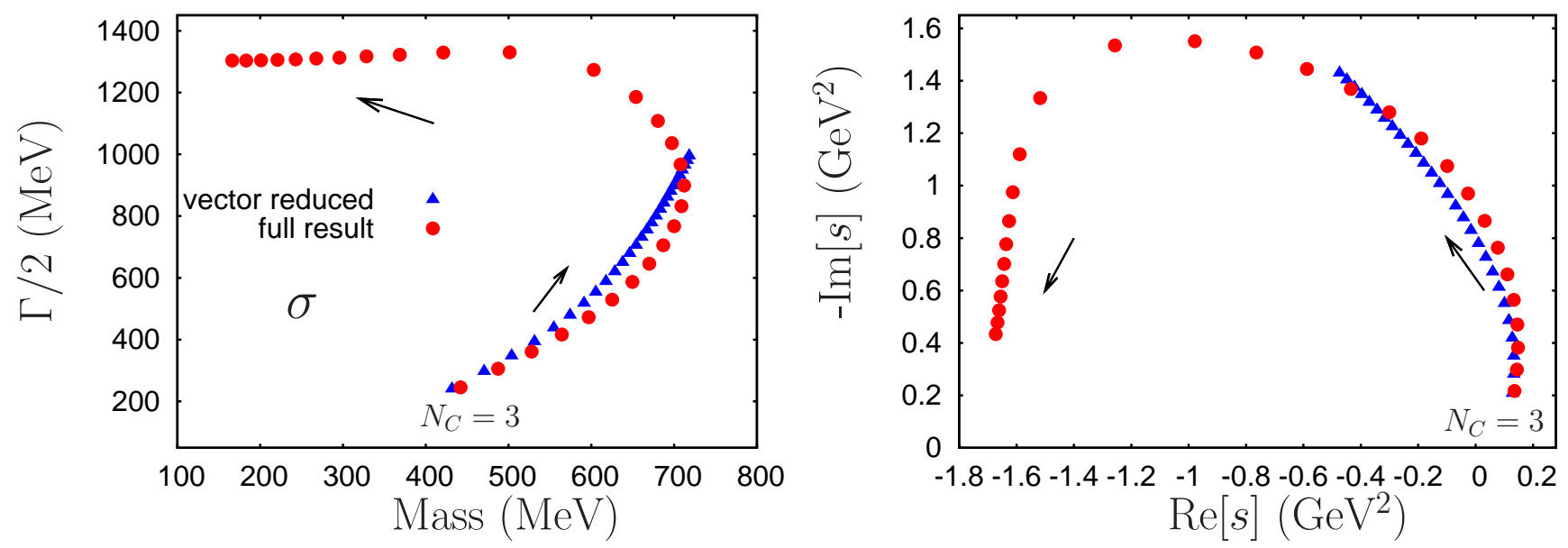

Figure 13: $N_{C}$ running of the pole positions for the $f_{0}(500)$ (or $\sigma$ ) resonance. We give the results for $N_{C}$ from 3 to 30 with one unit step. Both the full results and the ones from the vector reduced approximation are displayed. The left panel shows $\sqrt{s_{\sigma}}$ and the right one $s_{\sigma}$.

In Fig 13, we explicitly show the full (circles) and vector reduced (triangles) results for the $f_{0}(500)$ resonance pole $\left(s_{\sigma}\right)$ from $N_{C}=3$ up to $N_{C}=30$ in one unit step (as it will be also the case for the rest of pole trajectories). Though different strategies to estimate the sub-leading $N_{C}$ scaling for the resonance parameters have been used in the current work, the $N_{C}$ trajectories for the $f_{0}(500)$ are qualitatively consistent with those found in Ref. [1]. That is, the $f_{0}(500)$ resonance from the full calculation tends to fall down to the negative real axis in the $s_{\sigma}$-complex plane and moves further and further away along the nearby of the $s_{\sigma}$ negative axis as $N_{C}$ increases. For the vector reduced case this is not the case. The outcome from the latter resembles the $f_{0}(500)$ pole trajectory from the one-loop IAM $[42,44]$, and the full result trajectory is also one of the different $f_{0}(500)$ pole trajectories $[54,119]$. It is important to remark that both results, as well as the ones of Ref. [54], are compatible for values of $N_{C}<10$, and confirm again the results obtained in $[42,43]$, which predict a non dominant $\bar{q} q$ behavior for the $f_{0}(500)$. The outcome from the mimic $S U(3)$ approximation, which is not explicitly shown in the figure, is quite close to the full results, indicating the insensitivity of the $\sigma$ resonance to the $\eta$ and $\eta^{\prime}$ mesons, even when quite different masses result for these two particles as a function of $N_{C}$. As explained in Ref. [1] this relative insensitivity is due to the small couplings of the $f_{0}(500)$ to the $\eta \eta, \eta \eta^{\prime}$ and $\eta^{\prime} \eta^{\prime}$, even though these couplings are somewhat larger in the new fit than in the previous one. However, the largest couplings to $\pi \pi$ and $K \bar{K}$ are almost the same as in Ref. [1]. This pole trajectory clearly indicates that the $f_{0}(500)$ resonance has no significant $q \bar{q}$ or glueball components and it is in agreement with its dynamical generation from the isoscalar scalar $\pi \pi$ interactions. Ref. [38] calculates the quadratic scalar radius of the $\sigma,\left\langle r^{2}\right\rangle_{\sigma}^{S}=(0.19 \pm 0.02)-i(0.06 \pm 0.02) \mathrm{fm}^{2}$, so that it is concluded that this resonance is a compact one and the two pions merge inside it. As a result, a four-quark picture is more favorable than the molecular description.

On the other hand, the resulting pole trajectories of the heavier scalar resonances for the full result and the mimic $S U(3)$ approximation are quite different between each other, as one can see in Fig 14. This tells us that the scalar resonances $f_{0}(980), f_{0}(1370), K_{0}^{*}(1430)$ and $a_{0}(1450)$ are sensitive to the $\eta$ and $\eta^{\prime}$ states, to which they couple strongly as shown in Table 1. For the vector resonances, we show the $N_{C}$ trajectories in Fig 15, where the different treatments of the $\eta$ and $\eta^{\prime}$ mesons barely change the $\rho(770)$ resonance and only a little the $K^{*}(892)$. A kink structure has been found for the coupling strength between the $K^{*}(892)$ resonance and the $K \eta$ channel in the full result around $N_{C}=14$ [1], which is caused by the crossover of the $K^{*}(892)$ mass by the $K \eta$ threshold. This is mainly due to the variation of the $\eta$ mass. We find the structure from the new fit is quite similar to the one in Ref. [1]. For the $\phi(1020)$ and $\omega(780)$ resonances, we verify that it is also well behaved as a $\bar{q} q$ resonance at large $N_{C}$. The situation is even simpler in this case, since it only involves the $K \bar{K}$ channel in our approach. ${ }^{6}$ For the

\footnotetext{
${ }^{6}$ In the present study we are missing the important $3 \pi$ channel for a realistic treatment of the $\omega(780)$ resonance. We can then only study its mass which can be fixed to its experimental value by tuning its bare mass, as similarly done for the other vector resonances.
} 

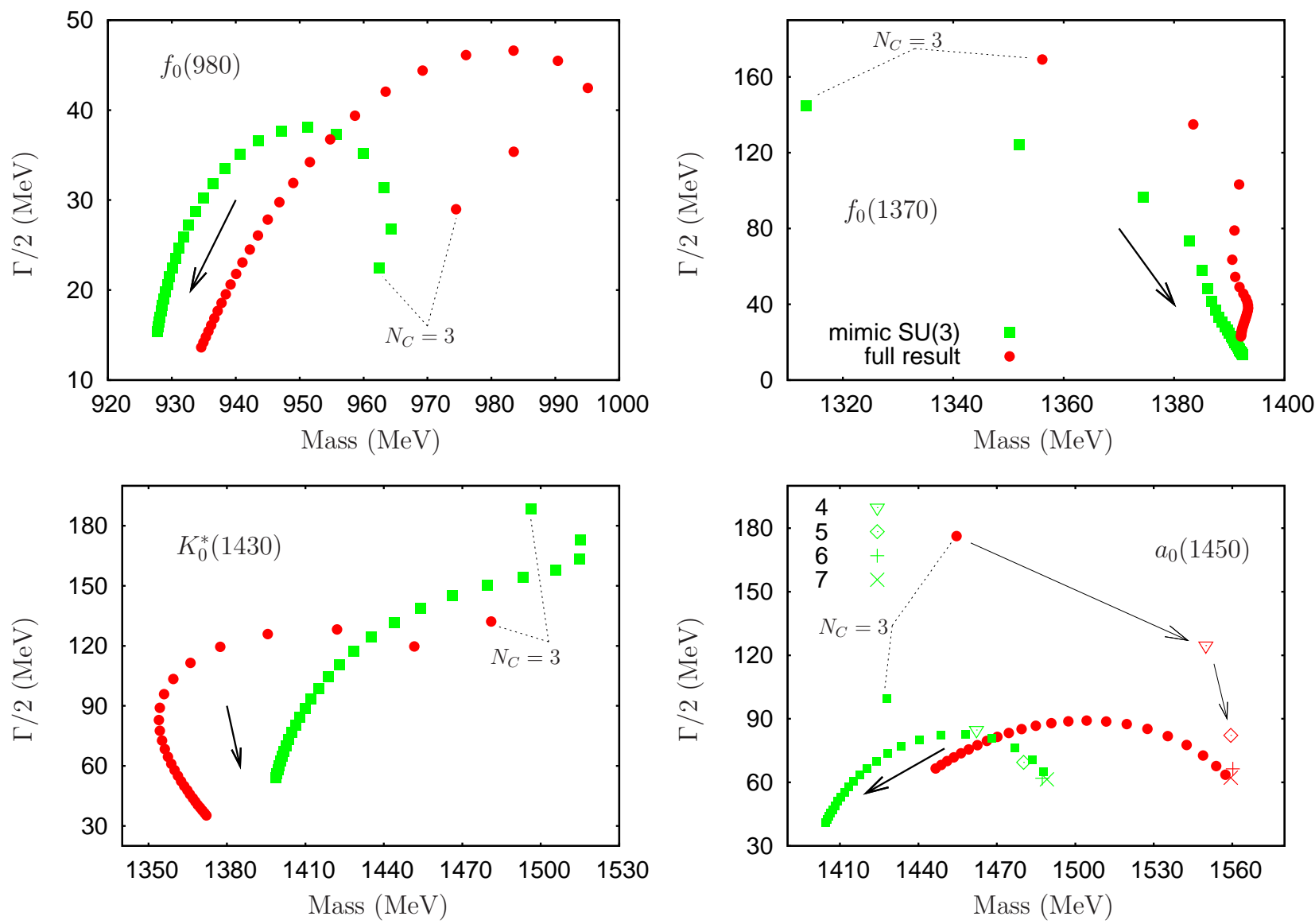

Figure 14: $N_{C}$ trajectories of the scalar resonances $f_{0}(980), f_{0}(1370), K_{0}^{*}(1430)$ and $a_{0}(1450)$. We show the results for $N_{C}$ from 3 to 30 with one unit step. The results for both the full (circles) and the mimic SU(3) (squares) calculations are shown. The differences between the two sets of trajectories show the sensitivity of the corresponding resonances to the $\eta$ and $\eta^{\prime}$ mesons. Specific symbols are assigned to the $a_{0}(1450)$ resonance for $N_{C}=4,5,6,7$ in order to show more clearly its pole trajectory.

$K_{0}^{*}(800)$ and $a_{0}(980)$ resonances, we re-confirm as in Ref. [1] that their pole positions go deeper and deeper in the complex energy plane when increasing $N_{C}$, which are displayed in Fig 16.

\subsection{Spectral-function sum rules for $N_{C}>3$}

As a further application, we study the $N_{C}$ evolution of the spectral functions and the spectral-function sum rules in this section. The scaling with $N_{C}$ for the two-point correlators, i.e. $W_{i}$ in Eq. (54), is proportional to $N_{C}$ in the large $N_{C}$ QCD [49]. The situation for the pseudoscalar correlators is obvious, since after substituting Eqs. (33), (B.11) and (B.12) into Eq. (54) one has

$$
W_{i} \propto F_{\pi}^{2}+8 d_{m}^{2}+\cdots, \quad i=P^{0}, P^{8}, P^{3},
$$

where the ellipsis stand both for sub-leading $N_{C}$ or suppressed pieces in the chiral counting. From Eq. (57) and the fact that $F_{\pi}$ scales as $\sqrt{N_{C}}$, it results that the leading $N_{C}$ scaling of the pseudoscalar correlators is proportional to $N_{C}$.

However, the situation for the scalar correlators is not so obvious. In fact, if we simply use the tree level results in Eqs. (B.6), (B.7) and (B.8) from the Lagrangian in Eq. (1) and substitute them into Eq. (19) to calculate the correlators in Eq. (54), one would conclude that the latter behave as a constant at large $N_{C}$, instead of running like 

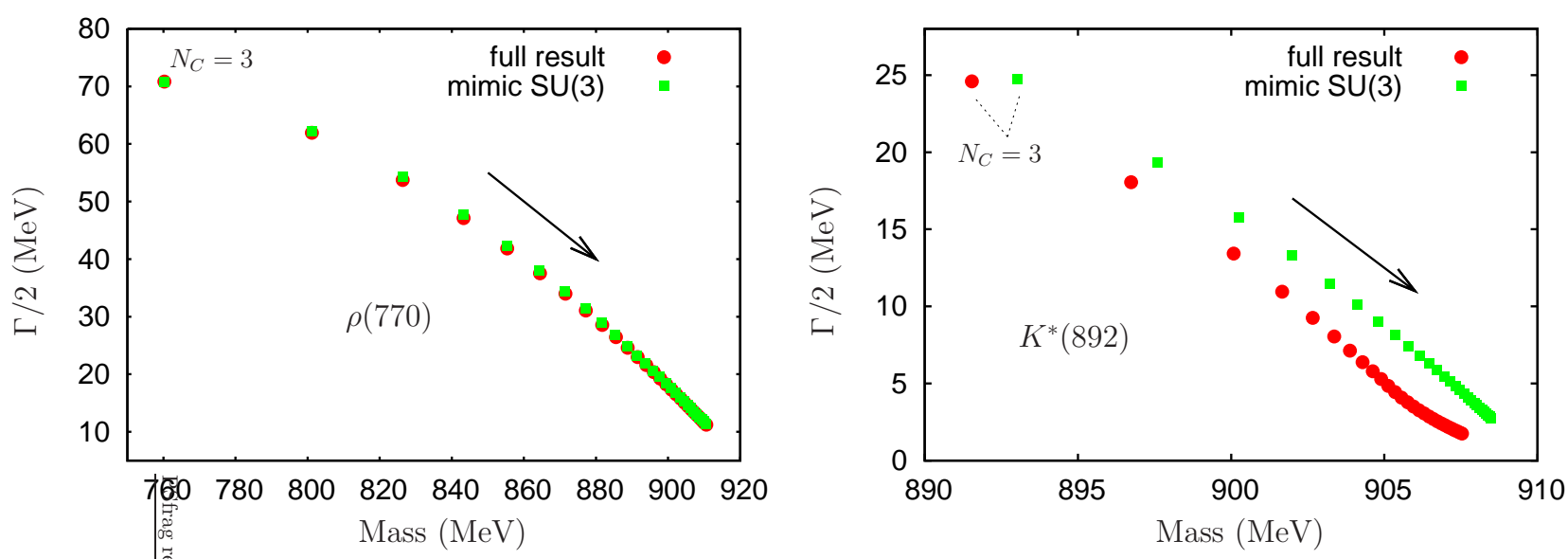

Figure 15: $N_{C}$ running of the pole positions for the $\rho(770)$ and $K^{*}(892)$ resonances. For notation see Fig. 14.
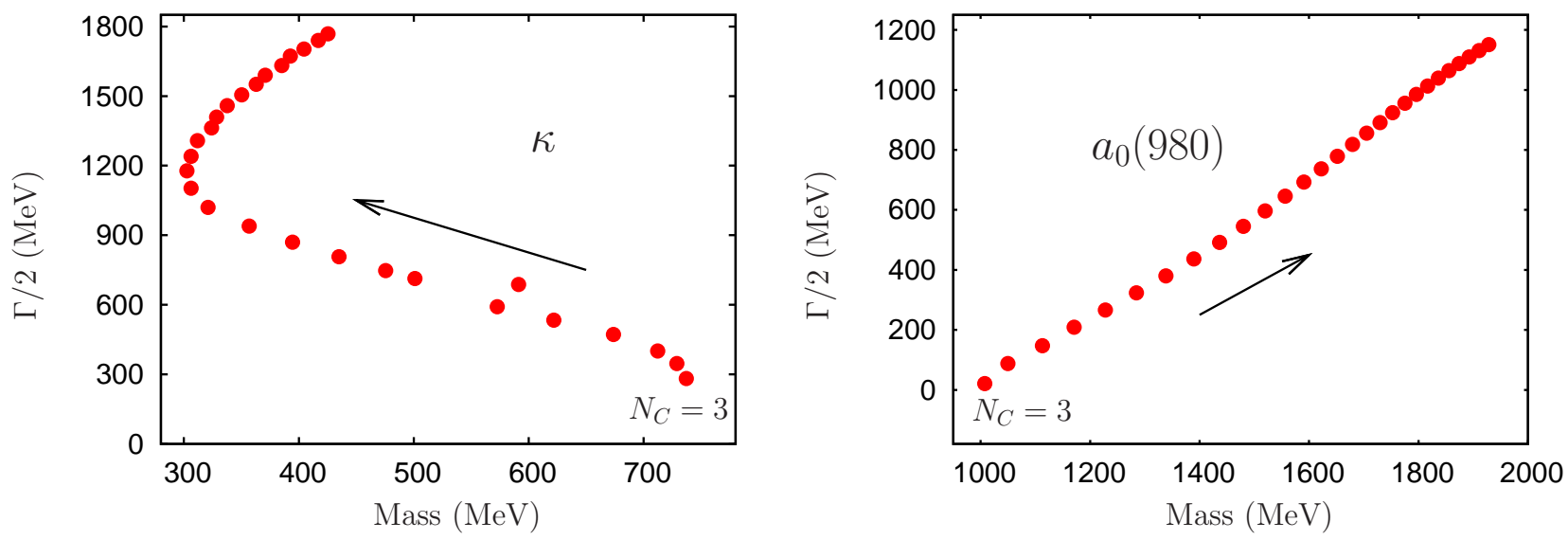

Figure 16: $N_{C}$ dependences of the $K_{0}^{*}(800)$ and $a_{0}(980)$ resonances both in the fourth Riemann sheets from $N_{C}=3$ to 30 with one unit step. See Ref. [1] for details on the definitions of the different Riemann sheets.

$N_{C}$. The subtlety, as shown in detail below, comes from the fact that at large $N_{C}$ the width of a $\bar{q} q$-like resonance tends to zero and the imaginary part of its propagator behaves then as a Dirac $\delta$-function, which is the dominant contribution to the correlators in Eq. (54).

In the following discussion, we demonstrate that by properly taking into account the resonance contributions in the unitarized scalar form factors Eq. (25) for the pseudo-Goldstone boson pairs, the scalar correlators in our formalism also scale like $N_{C}$, as required by large $N_{C}$ QCD. In order to make the analytical discussion neatly, we illustrate the proof in the chiral limit and consider the single channel case, i.e. we only include the $\pi \pi$ channel both for the scalar form factor and the scattering. The relevant parts $R^{I}(s), N^{I J}(s)$ and $g^{I J}(s)$ in Eq. (25) are just functions not matrices for the single channel. Notice also that in the large $N_{C}$ limit, only the tree level contributions, i.e. the (2)+Res parts in Eq. (26), can survive. We focus in the case $a=0$, while the others can be 
obtained analogously. The corresponding expressions in the chiral and large $N_{C}$ limits are:

$$
\begin{aligned}
R^{a=0}(s) & =-2-\frac{8 c_{d} c_{m}}{F^{2}} \frac{s}{M_{S}^{2}-s}, \\
N^{00}(s) & =\frac{s}{F^{2}}-\frac{G_{V}^{2}\left(2 M_{\rho}^{2}+3 s\right)}{F^{4}}+\frac{2 G_{V}^{2} M_{\rho}^{2}\left(M_{\rho}^{2}+2 s\right)}{F^{4} s} \log \left[1+\frac{s}{M_{\rho}^{2}}\right] \\
& +\frac{2 c_{d}^{2} M_{S}^{4}}{F^{4} s} \log \left[1+\frac{s}{M_{S}^{2}}\right]+\frac{c_{d}^{2}}{F^{4}} \frac{\left(2 s-M_{S}^{2}\right)\left(s+2 M_{S}^{2}\right)}{M_{S}^{2}-s}
\end{aligned}
$$

where we assume exact large $N_{C}$ nonet symmetry for the scalar resonances in Eqs. (4) and (8), i.e. imposing that the masses for singlet and octet scalar resonances are equal and taking the large $N_{C}$ relations in Eq. (44). In the previous equation $M_{S}$ denotes the bare mass of the scalar nonet. In fact for the scalar resonances, only the combination $\sqrt{\frac{1}{3}} S_{8}+\sqrt{\frac{2}{3}} S_{0}$ is the one relevant in the large $N_{C}$ limit for the $\pi \pi$ form factor and scattering. The interaction between the other orthogonal combination $\sqrt{\frac{1}{3}} S_{0}-\sqrt{\frac{2}{3}} S_{8}$ and $\pi \pi$ is $1 / N_{C}$ suppressed.

Let us concentrate in the $s$ region around the bare resonance pole at $s=M_{S}^{2}$ in Eqs. (77) and (78). Substituting Eqs. (77) and (78) into Eq. (25), we have the simple expression around the resonance pole $s \rightarrow M_{S}^{2}$ for the unitarized form factor

$$
F_{\pi \pi}^{a=0}(s)=-\frac{8 c_{d} c_{m} M_{S}^{2}}{F^{2}} \frac{1}{M_{S}^{2}-s-i \frac{3 c_{d}^{2} M_{S}^{4}}{16 \pi F^{4}}},
$$

where we have dropped the tiny contribution that stems from the real part of the function $g^{00}(s)$ (that would give rise to a self-energy contribution to the resonance bare mass), and explicitly show the contribution from the imaginary part in the chiral limit

$$
\operatorname{Im} g^{00}=-\frac{1}{16 \pi} .
$$

By substituting the tree level decay width of $S \equiv \sqrt{\frac{1}{3}} S_{8}+\sqrt{\frac{2}{3}} S_{0}$ to $\pi \pi$ calculated from the Lagrangian in Eq. (4) at the chiral limit [46]

$$
\Gamma_{S}=\frac{3 c_{d}^{2} M_{S}^{3}}{16 \pi F^{4}}
$$

into Eq. (79), we get the standard Breit-Wigner propagator for the scalar resonance

$$
F_{\pi \pi}^{a=0}(s)=-\frac{8 c_{d} c_{m} M_{S}^{2}}{F^{2}} \frac{1}{M_{S}^{2}-s-i M_{S} \Gamma_{S}} .
$$

Due to the fact that $\Gamma_{S}$ behaves as $1 / N_{C}$ in the large $N_{C}$ limit, we can use the standard narrow width approximation to write the form factor squared in terms of the Dirac $\delta$-function

$$
\begin{aligned}
\left|F_{\pi \pi}^{a=0}(s)\right|^{2} & =\frac{64 c_{d}^{2} c_{m}^{2} M_{S}^{4}}{F^{4}} \frac{1}{\left(M_{S}^{2}-s\right)^{2}+\left(M_{S} \Gamma_{S}\right)^{2}} \\
& \Rightarrow \frac{1024 \pi^{2}}{3} c_{m}^{2} \delta\left(s-M_{S}^{2}\right)
\end{aligned}
$$

where we have used Eq. (81) and the following way to approach the Dirac $\delta$-function:

$$
\frac{1}{\pi} \frac{M_{S} \Gamma_{S}}{\left(M_{S}^{2}-s\right)^{2}+\left(M_{S} \Gamma_{S}\right)^{2}} \Rightarrow \delta\left(s-M_{S}^{2}\right), \quad \text { when } M_{S} \Gamma_{S} \rightarrow 0
$$

By combining Eqs. (19), (83), (54) and (57) we obtain the leading $N_{C}$ behavior for the singlet two-point scalar correlator

$$
W_{S^{0}} \propto c_{m}^{2} \propto N_{C}
$$


which has the same scaling as the pseudoscalar case Eq. (76). This is important because otherwise we would run into the contradiction that it would not make sense to consider the spectral-function sum-rules as a function of $N_{C}$. The generalization to the cases with $a=3$ and 8 is straightforward.

We stress that we can make a close analytical discussion only at the leading order in $N_{C}$ and in the single channel case. Beyond these two conditions, it is rather difficult to perform the discussions analytically even in the chiral limit. Instead, our full calculations for the two-point correlators which include both the sub-leading $N_{C}$ parts, such as the chiral loops, and the multiple-channel dynamics, are carried out numerically. We show $W_{i} \times 3 / N_{C}$ in Fig. 17 for $i=S^{0}, S^{8}, S^{3}$ (left panel) and $P^{0}, P^{8}, P^{3}$ (right panel). The flat behavior at large $N_{C}$ clearly shows that all the two-point correlators $W_{i}$ are proportional to $N_{C}$ for large values of $N_{C}$. In addition, the asymptotic value is reached rather quickly with $N_{C}$, establishing already for $N_{C} \gtrsim 10$. The $W_{i}$ results are nearly the same at large values of $N_{C}$ either for the $S S$ correlators or the $P P$ ones as it is required by the spectral-function sum rules, Eqs. (17) (the discussion after Eq. (53) should be taken into account). To be more precise, we find that the relative variance $\sigma_{W} / \bar{W}$ for the six points at $N_{C}=30$ in Fig. 17 is only $5 \%$. This indicates that the spectral-function sum rules are better satisfied in large $N_{C}$ than for the $N_{C}=3$ case, reported in Table 3 with a relative variance of $10 \%$.

The reason behind this improvement can be attributed to the fact that at large $N_{C}$ the correlators reduce to the single resonance or pseudo-Goldstone exchanges and the large $N_{C}$ constraints from the $S S-P P$ sector requires that (as previously worked out in Ref. [20])

$$
c_{m}^{2}=d_{m}^{2}+\frac{F^{2}}{8}
$$

This is perfectly satisfied by the results from our research, with $c_{m}$ determined from the fit in Eq. (45), $d_{m}=30 \mathrm{MeV}$ adopted in this work $[41,20,17]$ and $F \simeq 80 \mathrm{MeV}$ predicted from the scalar resonance parameters in the fit Eq. (45) using the $U(3) \chi \mathrm{PT}$ one-loop calculation of Ref. [1].

It is also worth considering the evolution with $N_{C}$ of the scalar spectral functions. In the right panel of Fig. 8 the latter are shown for $N_{C}=30$. It is clear that the spectral functions are then completely dominated by the bare scalar pole $S_{1}$ and the octet of scalar resonances at around $\sqrt{s}=1.4 \mathrm{GeV}$. The low energy peaks in the left panel of Fig. 8 disappear for large $N_{C}$ due to its meson-meson dynamically generated nature, in agreement with what was already shown for the pole trajectories with $N_{C}$ in Sec. 6.2.

All the points in Fig. 17 are calculated by setting the upper limit of the integral in Eq. (54) to $s_{0}=3 \mathrm{GeV}^{2}$. We verify that the results are quite stable for $s_{0}=2.5 \mathrm{GeV}^{2}$ and $3.5 \mathrm{GeV}^{2}$, as it was already discussed for $N_{C}=3$ in Table 3 .

$W_{S^{a}}$

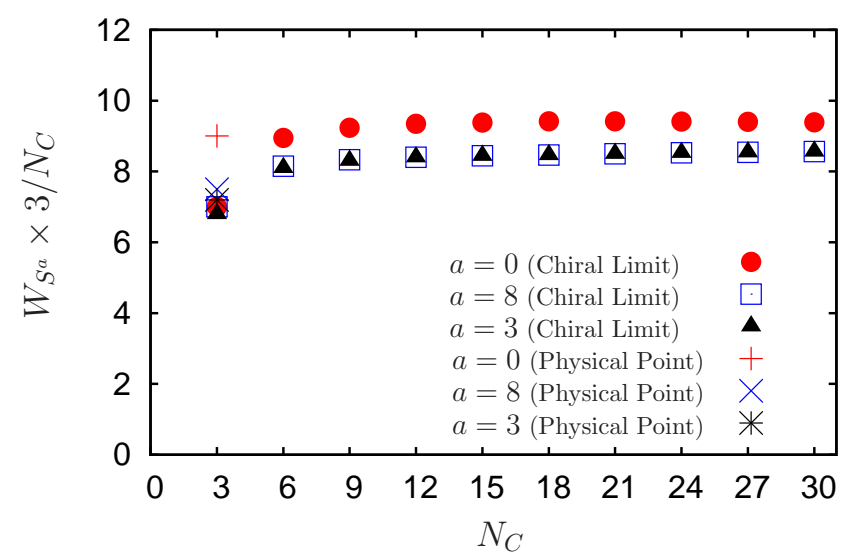

$W_{P^{a}}$

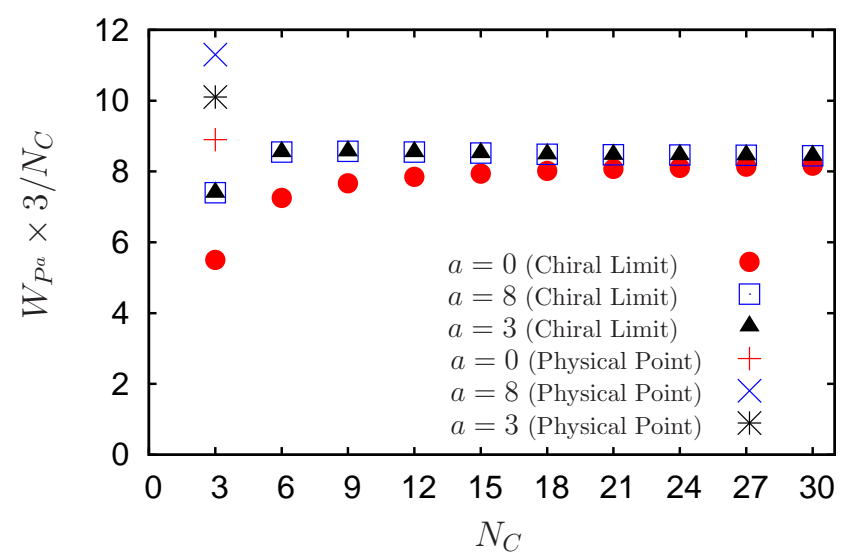

Figure 17: $W_{i} \times 3 / N_{C}$ as a function of $N_{C}$ within Scenario 3. We distinguish between the chiral limit and the physical case as indicated in the panels. We also show the corresponding value of $a$ for each point. 


\section{Conclusions}

In this work, we perform a complete one-loop calculation of the strangeness conserving scalar and pseudoscalar form factors within $U(3) \chi \mathrm{PT}$. We employ the resonance chiral Lagrangian to include the tree level resonance exchanges, instead of using the LECs from the local operators at higher chiral orders (which are supposed to be saturated by the resonance exchanges within the assumption of resonance saturation [41]). We extend the previous one-loop calculation on meson-meson scattering [1] by including the pseudoscalar resonance exchanges. The full results for the scalar form factors are calculated by using the unitarization method in Refs. [73, 72], based on the N/D method, taking as input the perturbative calculations of the scalar form factors and the partial wave scattering amplitudes. Then, we employ different form factors to calculate the various spectral functions, which allows us to study the spectral-function sum rules in the scalar and pseudoscalar sectors.

The unknown parameters in our theory are fitted to the experimental data. Comparing with the discussion in Ref. [1], a new fit is carried out in the present work, due to the inclusion of new ingredients, such as the pseudoscalar resonances and the second multiplet of scalar resonances. Various poles in the complex energy plane for the $f_{0}(500), f_{0}(980), f_{0}(1370), a_{0}(980), a_{0}(1450), K_{0}^{*}(800), K_{0}^{*}(1430), \rho(770), K^{*}(892)$ and $\phi(1020)$ resonances are found and they agree well with the values from PDG [36]. The coupling strengths of the various resonances to the pseudo-Goldstone boson pairs are also calculated. The resonance content of the new fit presented here and that of Ref. [1] is quite similar, both at the qualitative and quantitative levels. However, the new fit gives a better numerical value for the pion scalar radius than the one in Ref. [1]. The former also contains the pseudoscalar resonance exchanges which implies that the spectral-function sum rules in the scalar and pseudoscalar cases are satisfactorily fulfilled at the level of around a $10 \%$. In addition, the new fit results are employed to investigate semilocal duality in $\pi \pi$ scattering between Regge theory and the dynamics in terms of hadronic degree of freedom. We conclude that semilocal duality is well satisfied in general terms for $n \geq 1$.

An important advantage of working within the chiral Lagrangian approach is that it allows us to study the $N_{C}$ evolution of the various quantities calculated, once the $N_{C}$ scaling behavior of the parameters in the theory is settled. The leading evolution with $N_{C}$ is known unambiguously, while this is not the case for the sub-leading one. We propose three scenarios to take into account the uncertainties of the sub-leading $N_{C}$ scaling for the resonance parameters and another one to include the tensor resonances in the study of semilocal duality. Interestingly, we find that semilocal duality at large $N_{C}$ imposes strong constraints on the resonance parameters and Scenario 3 turns out to be the best one. Under this scenario we then study the extrapolation of $N_{C}>3$ for many other quantities: the masses of the pseudo-Goldstone bosons, the leading order $\eta-\eta^{\prime}$ mixing angle, the various resonance poles and the two-point correlators. We find that semilocal duality and the spectral-function sum rules in the scalar and pseudoscalar cases are well satisfied by using the parameters from the fit to experimental data, both for the physical case at $N_{C}=3$, and for varying $N_{C}>3$. It is important to stress that the fulfillment of semilocal duality and the spectral-function sum rules in the scalar and pseudoscalar sectors for both the physical and the large $N_{C}$ cases is not trivial and gives a deep insight in the isoscalar scalar spectrum for $\sqrt{s} \lesssim 1 \mathrm{GeV}$. In the physical case, the $f_{0}(500)$ resonance plays an important role for both semilocal duality and spectral-function sum rules, dominating the strength in the low-energy scalar isoscalar channel. However, when $N_{C}$ increases this resonance moves deeper in the complex energy plane and its contribution tends to vanish. Instead, the $f_{0}(980)$ resonance, which gradually evolves to the singlet scalar bare resonance $S_{1}$ when increasing $N_{C}$, plays the key role for the fulfillments of semilocal duality and spectral-function sum rules. It is then clear that the physical picture for the scalar isoscalar sector in the real and large $N_{C}$ worlds are very different. The former case is dominated by the $f_{0}(500)$ resonance, which should not have a significant $\bar{q} q$ or glueball components as it is clear from its $N_{C}$ pole trajectory. Within our approach it is dynamically generated by the $\pi \pi$ self-interactions. On the other hand, in the large $N_{C}$ limit the lightest resonance is the singlet $S_{1}$ bare or pre-existing resonance with a mass around 0.9-1.0 $\mathrm{GeV}$, which for $N_{C}=3$ is a component of the $f_{0}(980)$.

\section{Acknowledgements}

We would like to thank J. R. Peláez for interesting discussions. This work is partially funded by the grants MEC FPA2010-17806 and the Fundación Séneca 11871/PI/09. We also thank the financial support from the BMBF grant 06BN411, the EU-Research Infrastructure Integrating Activity "Study of Strongly Interacting Matter" 
(HadronPhysics2, grant No. 227431) under the Seventh Framework Program of EU and the Consolider-Ingenio 2010 Programme CPAN (CSD2007-00042). Z.H.G. acknowledges CPAN postdoc contract in the Universidad de Murcia and financial support from the grants National Natural Science Foundation of China (NSFC) under contract No. 11105038, Natural Science Foundation of Hebei Province with contract No. A2011205093 and Doctor Foundation of Hebei Normal University with contract No. L2010B04.

\section{Appendix}

\section{A Masses and mixing parameters}

In this section, we provide the contributions to the self-energies of the pseudo-Goldstone bosons that stem from the exchange of the pseudoscalar resonances, i.e. the operators in the last line of Eq. (10), and the $\delta L_{8}$ operator in Eq. (11). About the second multiplet of scalar resonances, its contributions share the same form as the lowest multiplet given in [1] with the obvious changes in couplings and masses. Hence, we do not reproduce them here. Notice that one needs to combine the results in the present section and those in the Appendices of Ref. [1] to get the full expressions.

We point out that the exchange of pseudoscalar resonances and the $\delta L_{8}$ operator do not contribute to the wave function renormalization of the pseudo-Goldstone bosons and the kinetic terms of $\bar{\eta}-\bar{\eta}^{\prime}$ mixing parameterized by $\delta_{\eta}, \delta_{\eta^{\prime}}$ and $\delta_{k}$ in Eq. (14) of Ref. [1]. For the pion and kaon masses these new contributions read

$$
\begin{aligned}
& m_{\pi}^{2}=\bar{m}_{\pi}^{2}+\frac{16 m_{\pi}^{4}}{F_{\pi}^{2}}\left(\delta L_{8}-\frac{d_{m}^{2}}{2 M_{P_{8}}^{2}}\right) \\
& m_{K}^{2}=\bar{m}_{K}^{2}+\frac{16 m_{K}^{4}}{F_{\pi}^{2}}\left(\delta L_{8}-\frac{d_{m}^{2}}{2 M_{P_{8}}^{2}}\right)
\end{aligned}
$$

The mass terms in the $\bar{\eta}-\bar{\eta}^{\prime}$ mixing are:

$$
\begin{aligned}
\delta_{m_{\bar{\eta}}}= & -\frac{8 d_{m}^{2}\left[c_{\theta}^{2}\left(m_{\pi}^{2}-4 m_{K}^{2}\right)^{2}+4 \sqrt{2} c_{\theta} s_{\theta}\left(4 m_{K}^{4}-5 m_{K}^{2} m_{\pi}^{2}+m_{\pi}^{4}\right)+8 s_{\theta}^{2}\left(m_{\pi}^{2}-m_{K}^{2}\right)^{2}\right]}{9 F_{\pi}^{2} M_{P_{8}}^{2}} \\
- & \frac{8 \widetilde{d}_{m}^{2}\left[8 c_{\theta}^{2}\left(m_{\pi}^{2}-m_{K}^{2}\right)^{2}+4 \sqrt{2} c_{\theta} s_{\theta}\left(2 m_{K}^{4}-m_{K}^{2} m_{\pi}^{2}-m_{\pi}^{4}\right)+s_{\theta}^{2}\left(m_{\pi}^{2}+2 m_{K}^{2}\right)^{2}\right]}{3 F_{\pi}^{2} M_{P_{1}}^{2}} \\
+ & \frac{16 \delta L_{8}}{3 F_{\pi}^{2}}\left[c_{\theta}^{2}\left(8 m_{K}^{4}-8 m_{K}^{2} m_{\pi}^{2}+3 m_{\pi}^{4}\right)+8 \sqrt{2} c_{\theta} s_{\theta} m_{K}^{2}\left(m_{K}^{2}-m_{\pi}^{2}\right)\right. \\
& \left.+s_{\theta}^{2}\left(4 m_{K}^{4}-4 m_{K}^{2} m_{\pi}^{2}+3 m_{\pi}^{4}\right)\right] \\
\delta_{m_{\bar{\eta}^{\prime}}^{2}}= & -\frac{8 d_{m}^{2}\left[8 c_{\theta}^{2}\left(m_{\pi}^{2}-m_{K}^{2}\right)^{2}-4 \sqrt{2} c_{\theta} s_{\theta}\left(4 m_{K}^{4}-5 m_{K}^{2} m_{\pi}^{2}+m_{\pi}^{4}\right)+s_{\theta}^{2}\left(m_{\pi}^{2}-4 m_{K}^{2}\right)^{2}\right]}{9 F_{\pi}^{2} M_{P_{8}}^{2}} \\
- & \frac{8 \widetilde{d}_{m}^{2}\left[c_{\theta}^{2}\left(m_{\pi}^{2}+2 m_{K}^{2}\right)^{2}-4 \sqrt{2} c_{\theta} s_{\theta}\left(2 m_{K}^{4}-m_{K}^{2} m_{\pi}^{2}-m_{\pi}^{4}\right)+8 s_{\theta}^{2}\left(m_{\pi}^{2}-m_{K}^{2}\right)^{2}\right]}{3 F_{\pi}^{2} M_{P_{1}}^{2}} \\
+ & \frac{16 \delta L_{8}}{3 F_{\pi}^{2}}\left[c_{\theta}^{2}\left(4 m_{K}^{4}-4 m_{K}^{2} m_{\pi}^{2}+3 m_{\pi}^{4}\right)-8 \sqrt{2} c_{\theta} s_{\theta} m_{K}^{2}\left(m_{K}^{2}-m_{\pi}^{2}\right)\right. \\
& \left.+s_{\theta}^{2}\left(8 m_{K}^{4}-8 m_{K}^{2} m_{\pi}^{2}+3 m_{\pi}^{4}\right)\right]
\end{aligned}
$$




$$
\begin{aligned}
\delta_{m^{2}}= & \frac{8 d_{m}^{2}}{9 F_{\pi}^{2} M_{P_{8}}^{2}}\left[2 \sqrt{2} c_{\theta}^{2}\left(4 m_{K}^{4}-5 m_{K}^{2} m_{\pi}^{2}+m_{\pi}^{4}\right)+c_{\theta} s_{\theta}\left(-8 m_{K}^{4}-8 m_{K}^{2} m_{\pi}^{2}+7 m_{\pi}^{4}\right)\right. \\
& \left.-2 \sqrt{2} s_{\theta}^{2}\left(4 m_{K}^{4}-5 m_{K}^{2} m_{\pi}^{2}+m_{\pi}^{4}\right)\right] \\
+ & \frac{8 \widetilde{d}_{m}^{2}}{3 F_{\pi}^{2} M_{P_{1}}^{2}}\left[2 \sqrt{2} c_{\theta}^{2}\left(2 m_{K}^{4}-m_{K}^{2} m_{\pi}^{2}-m_{\pi}^{4}\right)+c_{\theta} s_{\theta}\left(-4 m_{K}^{4}+20 m_{K}^{2} m_{\pi}^{2}-7 m_{\pi}^{4}\right)\right. \\
+ & \left.2 \sqrt{2} s_{\theta}^{2}\left(-2 m_{K}^{4}+m_{K}^{2} m_{\pi}^{2}+m_{\pi}^{4}\right)\right] \\
+ & \frac{64 \delta L_{8} m_{K}^{2}\left(m_{\pi}^{2}-m_{K}^{2}\right)\left(\sqrt{2} c_{\theta}^{2}-c_{\theta} s_{\theta}-\sqrt{2} s_{\theta}^{2}\right)}{3 F_{\pi}^{2}},
\end{aligned}
$$

where $\delta_{m \frac{2}{\eta}}, \delta_{m_{\eta^{\prime}}}$ and $\delta_{m^{2}}$ are defined in Eq. (14) of Ref. [1]. Notice that the two operators in the last line of the pseudoscalar resonance Lagrangian in Eq. (10) and the $\delta L_{8}$ operator of Eq. (11) do not contribute to the pion decay constant $F_{\pi}$.

\section{B Analytic expressions for scalar and pseudoscalar form factors}

In order to calculate the scalar form factors in the isospin basis of Eqs. (21) and (22), we need to evaluate for the isoscalar case eight form factors in the charged bases, namely: $F_{\pi^{0} \pi^{0}}^{0,8}, F_{\pi^{+} \pi^{-}}^{0,8}, F_{\pi^{-} \pi^{+}}^{0,8}, F_{K^{+} K^{-}}^{0,8}, F_{K^{0} \bar{K}^{0}}^{0,8}, F_{\eta \eta}^{0,8}, F_{\eta \eta^{\prime}}^{0,8}$ and $F_{\eta^{\prime} \eta^{\prime}}^{0,8}$. For the isovector case, one needs: $F_{\pi^{0} \eta}^{3}, F_{K^{+} K^{-}}^{3}, F_{K^{0}}^{3} \bar{K}^{0}$ and $F_{\pi^{0} \eta^{\prime}}^{3}$

The leading order contributions to the scalar form factors defined in Eq. (18) stem from the Lagrangian in Eq. (1). The expressions for $a=0$ read:

$$
\begin{aligned}
F_{\pi^{0} \pi^{0}}^{0} & =F_{\pi^{+} \pi^{-}}^{0}=F_{\pi^{-} \pi^{+}}^{0}=2 \sqrt{\frac{2}{3}}, \\
F_{K^{+} K^{-}}^{0} & =F_{K^{0} \bar{K}^{0}}^{0}=2 \sqrt{\frac{2}{3}}, \\
F_{\eta \eta}^{0} & =2 \sqrt{\frac{2}{3}} \\
F_{\eta \eta^{\prime}}^{0} & =0 \\
F_{\eta^{\prime} \eta^{\prime}}^{0} & =2 \sqrt{\frac{2}{3}} .
\end{aligned}
$$

For $a=8$ one has:

$$
\begin{aligned}
F_{\pi^{0} \pi^{0}}^{8} & =F_{\pi^{+} \pi^{-}}^{8}=F_{\pi^{-} \pi^{+}}^{8}=\frac{2}{\sqrt{3}}, \\
F_{K^{+} K^{-}}^{8} & =F_{K^{0} \bar{K}^{0}}^{8}=-\frac{1}{\sqrt{3}}, \\
F_{\eta \eta}^{8} & =-\frac{2 c_{\theta}\left(c_{\theta}+2 \sqrt{2} s_{\theta}\right)}{\sqrt{3}}, \\
F_{\eta \eta^{\prime}}^{8} & =\frac{2\left(\sqrt{2} c_{\theta}^{2}-c_{\theta} s_{\theta}-\sqrt{2} s_{\theta}^{2}\right)}{\sqrt{3}}, \\
F_{\eta^{\prime} \eta^{\prime}}^{8} & =\frac{2 s_{\theta}\left(2 \sqrt{2} c_{\theta}-s_{\theta}\right)}{\sqrt{3}} .
\end{aligned}
$$


And the results for $a=3$ are:

$$
\begin{aligned}
F_{\pi^{0} \eta}^{3} & =\frac{2\left(c_{\theta}-\sqrt{2} s_{\theta}\right)}{\sqrt{3}}, \\
F_{K^{+} K^{-}}^{3} & =-F_{K^{0} \bar{K}^{0}}^{3}=1, \\
F_{\pi^{0} \eta^{\prime}}^{3} & =\frac{2\left(\sqrt{2} c_{\theta}+s_{\theta}\right)}{\sqrt{3}} .
\end{aligned}
$$

The scalar form factors in the quark flavor basis defined in Eqs. (29) at leading order are

$$
\begin{aligned}
& F_{\pi^{0} \pi^{0}}^{\bar{u} u+\bar{d} d}=F_{\pi^{+} \pi^{-}}^{\bar{u} u+\bar{d} d}=F_{\pi^{-} \pi^{+}}^{\bar{u} u+\bar{d} d}=2, \\
& F_{K^{+} K^{-}}^{\bar{u} u+\bar{d} d}=F_{K^{0} \bar{K}^{0}}^{\bar{u} u+\bar{d} d}=1, \\
& F_{\eta \eta}^{\bar{u} u+\bar{d} d}=-\frac{2}{3}\left(c_{\theta}^{2}+2 \sqrt{2} c_{\theta} s_{\theta}-2\right), \\
& F_{\eta \eta^{\prime}}^{\bar{u} u+\bar{d} d}=\frac{2}{3}\left(\sqrt{2} c_{\theta}^{2}-c_{\theta} s_{\theta}-\sqrt{2} s_{\theta}^{2}\right), \\
& F_{\eta^{\prime} \eta^{\prime}}^{\bar{u} u+\bar{d} d}=\frac{2}{3}\left(-s_{\theta}^{2}+2 \sqrt{2} c_{\theta} s_{\theta}+2\right),
\end{aligned}
$$

and

$$
\begin{aligned}
F_{\pi^{0} \pi^{0}}^{\bar{s} s} & =F_{\pi^{+} \pi^{-}}^{\bar{s} s}=F_{\pi^{-} \pi^{+}}^{\bar{s} s}=0 \\
F_{K^{+} K^{-}}^{\bar{s} s} & =F_{K^{0} \bar{K}^{0}}^{\bar{s} s}=1 \\
F_{\eta \eta}^{\bar{s} s} & =\frac{2}{3}\left(c_{\theta}^{2}+2 \sqrt{2} c_{\theta} s_{\theta}+1\right), \\
F_{\eta \eta^{\prime}}^{\bar{s} s} & =-\frac{2}{3}\left(\sqrt{2} c_{\theta}^{2}-c_{\theta} s_{\theta}-\sqrt{2} s_{\theta}^{2}\right), \\
F_{\eta^{\prime} \eta^{\prime}}^{\bar{s} s} & =\frac{2}{3}\left(s_{\theta}^{2}-2 \sqrt{2} c_{\theta} s_{\theta}+1\right) .
\end{aligned}
$$

At the same order, the pseudoscalar form factors for the pseudo-Goldstone bosons defined in Eq. (34) are

$$
\begin{array}{rcl}
H_{\pi^{0}}^{0}=0, & H_{\eta}^{0}=-2 F_{\pi} s_{\theta}, & H_{\eta^{\prime}}^{0}=2 F_{\pi} c_{\theta}, \\
H_{\pi^{0}}^{8}=0, & H_{\eta}^{8}=2 F_{\pi} c_{\theta}, & H_{\eta^{\prime}}^{8}=2 F_{\pi} s_{\theta}, \\
H_{\pi^{0}}^{3}=2 F_{\pi}, & H_{\eta}^{3}=0, & H_{\eta^{\prime}}^{3}=0,
\end{array}
$$

and for the pseudoscalar resonances they read

$$
\begin{aligned}
& H_{\text {Pseudoscalar Resonances }}^{0}=-4 \sqrt{6} \widetilde{d}_{m}, \\
& H_{\text {Pseudoscalar Resonances }}^{8}=-4 \sqrt{2} d_{m}, \\
& H_{\text {Pseudoscalar Resonances }}^{3}=-4 \sqrt{2} d_{m} .
\end{aligned}
$$

We provide the remaining expressions, such as those from the chiral loops, scalar resonances, pseudoscalar resonances, $\Lambda_{2}$ and $\delta L_{8}$, in the Mathematica code [76].

\section{Tensor resonances in meson-meson scattering}

We follow the framework proposed in Ref. [105] to include the tensor resonances in meson-meson scattering. The relevant Lagrangian reads

$$
\mathcal{L}_{T}=-\frac{1}{2}\left\langle T_{\mu \nu} D_{\mathrm{T}}^{\mu \nu, \rho \sigma} T_{\rho \sigma}\right\rangle+g_{T}\left\langle T_{\mu \nu}\left\{u^{\mu}, u^{\nu}\right\}\right\rangle+\beta\left\langle T_{\mu}^{\mu} u_{\nu} u^{\nu}\right\rangle+\gamma\left\langle T_{\mu}^{\mu} \chi_{+}\right\rangle
$$


where the nonet of the tensor resonances are collected in the matrix

$$
T_{\mu \nu}=\left(\begin{array}{ccc}
\frac{a_{2}^{0}}{\sqrt{2}}+\frac{f_{2}^{8}}{\sqrt{6}}+\frac{f_{2}^{1}}{\sqrt{3}} & a_{2}^{+} & K_{2}^{*+} \\
a_{2}^{-} & -\frac{a_{2}^{0}}{\sqrt{2}}+\frac{f_{2}^{8}}{\sqrt{6}}+\frac{f_{2}^{1}}{\sqrt{3}} & K_{2}^{* 0} \\
K_{2}^{*-} & \bar{K}_{2}^{* 0} & -\frac{2 f_{2}^{8}}{\sqrt{6}}+\frac{f_{2}^{1}}{\sqrt{3}}
\end{array}\right)_{\mu \nu} .
$$

See Ref. [41] for the definition of the remaining chiral building blocks. Like in the vector resonance case [1], ideal mixing is also assumed for the tensor resonances:

$$
\begin{aligned}
f_{2}(1270) & =\sqrt{\frac{2}{3}} f_{2}^{1}+\sqrt{\frac{1}{3}} f_{2}^{8}, \\
f_{2}^{\prime} & =\sqrt{\frac{1}{3}} f_{2}^{1}-\sqrt{\frac{2}{3}} f_{2}^{8} .
\end{aligned}
$$

In the present work, the purpose to introduce the tensor resonances is to discuss semilocal duality in $\pi \pi$ scattering. So we will set equal all the tensor masses, which is determined by the most relevant resonance $f_{2}(1270)$. The first operator in Eq. (C.13) corresponds to the kinetic term while the remaining terms describe the interactions between the tensor resonances and the pseudo-Goldstone boson pairs. The terms proportional to $\beta$ and $\gamma$ do not contribute to the on-shell decay of the tensor resonances because the tensor field is traceless in the space-time indices [105]. Nevertheless, they do contribute to meson-meson scattering. It is argued in Ref. [105] that though the final result is independent of the choice of $\beta$, it is convenient to set $\beta=-g_{T}$ to avoid the inclusion of the $\mathcal{O}\left(p^{6}\right)$ LECs in order to fulfill the high energy constraints for the forward $\pi \pi$ scattering. The $\gamma$ operator is always accompanied by quark masses and its effects should be much less important than the $\beta$ term. So we will omit the former term throughout as done in Ref. [105].

Last but not least, one should guarantee the right high energy behavior of meson-meson scattering in the presence of tensor resonances resulting from the Lagrangian in Eq. (C.13). The high energy constraint imposed in Ref. [105] concerns the fulfillment of a once subtracted forward dispersion relation for $\pi^{+} \pi^{0}$ elastic scattering, which turns out to play a crucial role to get the correct prediction for the LECs. We follow the same approach here to satisfy this high energy constraint. Moreover we calculate all the relevant meson-meson coupled channels for scattering in $U(3) \chi \mathrm{PT}$, not just $\pi \pi$, since the other processes can enter through the unitarization procedure [1] that couples the different states with the same quantum numbers.

The final results on meson-meson scattering contributed by the tensor resonances and the high energy constraints are given in the Mathematica code [76]. Due to the consideration of the scattering processes involving $\eta$ and $\eta^{\prime}$, additional LECs are needed to guarantee the proper short distance constraint imposed by the forward scattering. The short distance constraints that we find here are

$$
\beta_{13}^{S D}=\frac{4 g_{T}^{2}}{M_{T}^{2}}, \quad \beta_{14}^{S D}=-\frac{g_{T}^{2}}{M_{T}^{2}}, \quad \beta_{15}^{S D}=-\frac{2 g_{T}^{2}}{M_{T}^{2}}, \quad \beta_{16}^{S D}=-\frac{g_{T}^{2}}{2 M_{T}^{2}},
$$

in addition to the ones determined in Ref. [105]

$$
\beta_{1}^{S D}=-\frac{g_{T}^{2}}{2 M_{T}^{2}}, \quad \beta_{2}^{S D}=-\frac{g_{T}^{2}}{M_{T}^{2}}, \quad \beta_{3}^{S D}=\frac{2 g_{T}^{2}}{M_{T}^{2}} .
$$

The convention to label the LECs in Eq. (C.16) is the same as in Ref. [120], where one can also find the corresponding monomials multiplied by the coefficients with $i=1,2,3,13,14,15$, and 16 . We checked that only after the short distance constraints are fulfilled, the introduction of the tensor resonances does not spoil the fit that we obtained in Ref. [1] and the new one in Eq. (45).

For the value of $g_{T}$, we take the result $g_{T}=28 \mathrm{MeV}$ from Ref. [105]. As in the vector channels [1], due to the dominant role played by the tree level tensor resonances, one does not expect the subtraction constants arising from the unitarization procedure to play an important role in $I J=02$. We simply fix their values to the $I J=00$ channel and checked that the results are stable under $\mathcal{O}(1)$ changes in these numbers, so that the subtraction constants keep natural values in Eq. (45). The bare mass of the tensor resonances is adjusted to $M_{T}=1300 \mathrm{MeV}$, leading to the pole position $\sqrt{s}=(1275.2-i 75.8) \mathrm{MeV}$, which is close to the values of the $f_{2}(1270)$ resonance given in the PDG [36]. 


\section{References}

[1] Zhi-Hui Guo and J.A. Oller, Phys. Rev. D 84 (2011) 034005.

[2] S. Weinberg, Physica A 96 (1979) 327.

[3] J. Gasser and H. Leutwyler, Annals Phys. 158 (1984) 142.

[4] J. Gasser and H. Leutwyler, Nucl. Phys. B 250 (1985) 465.

[5] K. G. Wilson, Phys. Rev. 179 (1969) 1499.

[6] P. Pascual and R. Tarrach, QCD: Renormalization for the practitioner. Springer-Verlag, Berlin Heidelberg, 1984.

[7] S. Weinberg, Phys. Rev. Lett. 18 (1967) 507.

[8] For a recent review: M. Davier, A. Hocker and Z. Zhang, Rev. Mod. Phys. 78 (2006) 1043.

[9] S. Narison, N. Paver, E. de Rafael and D. Treleani, Nucl. Phys. B 212 (1983) 365.

[10] M. Jamin and M. Munz, Z. Phys. C 60 (1993) 569.

[11] P. A. Baikov and K. G. Chetyrkin, Phys. Rev. Lett. 97 (2006) 061803.

[12] B. Moussallam, Eur. Phys. J. C 14 (2000) 111.

[13] B. Moussallam, JHEP 0008 (2000) 005.

[14] S. Peris, M. Perrottet and E. de Rafael, JHEP 9805 (1998) 011.

[15] J. Bijnens, E. Gamiz and J. Prades, JHEP 0110 (2001) 009.

[16] J. Bijnens, E. Gamiz and J. Prades, JHEP 0304 (2003) 055.

[17] J. J. Sanz-Cillero and J. Trnka, Phys. Rev. D 81 (2010) 056005.

[18] M. Jamin, J. A. Oller and A. Pich, Eur. Phys. J. C 24 (2002) 237; Phys. Rev. D 74 (2006) 074009.

[19] A. Dominguez-Clarimon, E. de Rafael and J. Taron, Phys. Lett. B 660 (2008) 49.

[20] M. F. L. Golterman and S. Peris, Phys. Rev. D 61 (2000) 034018.

[21] M. Jamin, J. A. Oller and A. Pich, JHEP 0402 (2004) 047.

[22] M. Jamin, J. A. Oller and A. Pich, Nucl. Phys. B 587 (2000) 331.

[23] M. Jamin, J. A. Oller and A. Pich, Nucl. Phys. B 622 (2002) 279.

[24] N. Kaiser, P. B. Siegel and W. Weise, Nucl. Phys. A 594 (1995) 325.

[25] J. Oller and E. Oset, Nucl. Phys. A 620 (1997) 438; (E)-ibid A 652 (1999) 407.

[26] J. A. Oller and E. Oset, Phys. Rev. D 60 (1999) 074023.

[27] R. Kaminski, J. R. Peláez and F. J. Ynduráin, Phys. Rev. D 77 (2008) 054015; R. Garcia-Martin, R. Kaminski, J. R. Peláez, J. Ruiz de Elvira and F.J. Yndurain, Phys. Rev. D 83 (2011) 074004.

[28] J. Nieves, M. Pavón Valderrama and E. Ruiaz Arriola, Phys. Rev. D 65 (2002) 036002.

[29] I. Caprini, G. Colangelo and H. Leutwyler, Phys. Rev. Lett. 96 (2006) 132001.

[30] S. Descotes-Genon and B. Moussallam, Eur. Phys. J. C 48 (2006) 553. 
[31] Z. Xiao and H. Q. Zheng, Nucl. Phys. A 695 (2001) 273.

[32] H. Q. Zheng, Z. Y. Zhou, G. Y. Qin, Z. Xiao, J. J. Wang and N. Wu, Nucl. Phys. A 733 (2004) 235.

[33] Z. -Y. Zhou and Z. Xiao, Phys. Rev. D 83 (2011) 014010.

[34] J. Nieves, A. Pich and E. Ruiz Arriola, Phys. Rev. D 84 (2011) 096002.

[35] M. Albaladejo and J. A. Oller, Phys. Rev. Lett. 101 (2008) 252002.

[36] J. Beringer et al. (Particle Data Group), Phys. Rev. D 86 (2012) 010001.

[37] Z.-H. Guo, J. A. Oller and J. Ruiz de Elvira, Phys. Lett. B 712 (2012) 407.

[38] M. Albaladejo and J. A. Oller, Phys. Rev. D 86 (2012) 034003.

[39] C. W. Bernard, A. Duncan, J. LoSecco and S. Weinberg, Phys. Rev. D 12 (1975) 792.

[40] H. Leutwyler, Nucl. Phys. B 337 (1990) 108.

[41] G. Ecker, J. Gasser, A. Pich and E. de Rafael, Nucl. Phys. B 321 (1989) 311.

[42] J. R. Peláez, Phys. Rev. Lett. 92 (2004) 102001.

[43] J. R. Peláez and G. Rios, Phys. Rev. Lett. 97 (2006) 242002.

[44] Z. X. Sun, L. Y. Xiao, Z. Xiao and H.Q. Zheng, Mod. Phys. Lett. A 22 (2007) 711.

[45] L. Y. Xiao, Z.-H. Guo and H. Q. Zheng, Int. J. Mod. Phys. A 22 (2007) 4603.

[46] Z.-H. Guo, J. J. Sanz-Cillero and H.-Q. Zheng, JHEP 06 (2007) 030.

[47] J. Nieves and E. Ruiz Arriola, Phys. Rev. D 80 (2009) 045023.

[48] L. Y. Dai, X. G. Wang and H. Q. Zheng, Commun. Theor. Phys. 57 (2012) 841.

[49] G. 't Hooft, Nucl. Phys. B 72 (1974) 461; E. Witten, Nucl. Phys. B 160 (1979) 57.

[50] A. V. Manohar, hep-ph/9802419.

[51] E. Witten, Nucl. Phys. B 156 (1979) 269; S. Coleman and E. Witten, Phys. Rev. Lett. 45 (1980) 100; G. Veneziano, Phys. Lett. B 95 (1980) 90.

[52] S. Weinberg, Phys. Rev. D 11 (1975) 3583.

[53] P.D.B. Collins, An introduction to Regge theory and high energy physics. Cambridge University Press, Cambridge, 1977.

[54] J. R. Peláez, M. R. Pennington, J. Ruiz de Elvira and D. J. Wilson, Phys. Rev. D 84 (2011) 096006.

[55] T. N. Truong, Phys. Rev. Lett. 61 (1988) 2526.

[56] A. Dobado, M. J. Herrero and T. N. Truong, Phys. Lett. B 235 (1990) 134.

[57] A. Dobado and J. R. Peláez, Phys. Rev. D 56 (1997) 3057.

[58] J. A. Oller, E. Oset and J. R. Peláez, Phys. Rev. Lett. 80 (1998) 3452; Phys. Rev. D 59 (1999) 074001; (E)-ibid D 60 (1999) 099906; (E)-ibid D 75 (2007) 099903.

[59] F. J. Llanes-Estrada, J. R. Pelaez and J. Ruiz de Elvira, Nucl. Phys. Proc. Suppl. 207-208 (2010) 169.

[60] P. Di Vecchia and G. Veneziano, Nucl. Phys. B 171 (1980) 253; C. Rosenzweig, J. Schechter and T. Trahem, Phys. Rev. D 21 (1980) 3388; E. Witten, Ann. Phys. 128 (1980) 363. 
[61] K. Karawabayashi and N. Ohta, Nucl. Phys. B 175 (1980) 477; Prog. Theor. Phys. 66 (1981) 1789.

[62] J. A. Oller and U.-G. Meißner, Phys. Lett. B 500 (2001) 263.

[63] W. A. Bardeen, Phys. Rev. 184 (1969) 1848.

[64] K. Fujikawa, Phys. Rev. D 21 (1980) 2848.

[65] S. L. Adler and W. A. Bardeen, Phys. Rev. 182 (1969) 1517.

[66] G.'t Hooft, Phys. Rev. D 14 (1976) 3432; Phys. Rep. 142 (1986) 357.

[67] G. Ecker, J. Gasser, H. Leutwyler, A. Pich and E. de Rafael, Phys. Lett. B 223 (1989) 425.

[68] M. Albaladejo, J. A. Oller and L. Roca, Phys. Rev. D 82 (2010) 094019.

[69] R. Kaiser and H. Leutwyler, Eur. Phys. J. C 17 (2000) 623.

[70] A. Pich, I. Rosell and J. J. Sanz-Cillero, JHEP 0807 (2008) 014.

[71] S. Weinberg, Nucl. Phys. B 363 (1991) 3.

[72] U. -G. Meißner and J. A. Oller, Nucl. Phys. A 679 (2001) 671.

[73] J. A. Oller, E. Oset and J. E. Palomar, Phys. Rev. D 63 (2001) 114009.

[74] J. A. Oller, Phys. Rev. D 71 (2005) 054030.

[75] http://www.um.es/oller/u3FullAmp16.nb

[76] http://www.um.es/oller/AmpFF.nb

[77] J. Gasser and H. Leutwyler, Nucl. Phys. B 250 (1985) 517.

[78] J. F. Donoghue, J. Gasser and H. Leutwyler, Nucl. Phys. B 343 (1990) 341.

[79] J. A. Oller, Phys. Lett. B 477 (2000) 187.

[80] U.-G. Meißner and J. A. Oller, Nucl. Phys. A 673 (2000) 311.

[81] J. A. Oller, E. Oset and A. Ramos, Prog. Part. Nucl. Phys. 45 (2000) 157.

[82] G. Colangelo, J. Gasser and H. Leutwyler, Nucl. Phys. B 603 (2001) 125.

[83] J. A. Oller and L. Roca, Phys. Lett. B 651 (2007) 139.

[84] Z. H. Guo and J. J. Sanz-Cillero, Phys. Rev. D 79 (2009) 096006.

[85] J. Bijnens and I. Jemos, Nucl. Phys. B 854 (2012) 631.

[86] C. D. Frogratt and J. L. Petersen, Nucl. Phys. B 129 (1977) 89.

[87] J. R. Batley et al. (NA48/2 Collaboration), Eur. Phys. J. C 54 (2008) 411.

[88] W. Ochs, Ph.D. Thesis, University of Munich , 1974.

[89] B. Hyams, et al., Nucl. Phys. B 64 (1973) 134; P. Estabrooks, et al., AIP Conf. Proc. 13 (1973) 37; G. Grayer, et al., Proceedings of the 3rd Philadephia Conference on Experimental Meson Spectroscopy, Philadephia, 1972 (American Institute of Physics, New York, 1972), p.5; S. D. Protopopescu and M. Alson-Garnjost, Phys. ReV. D 7 (1973) 1279.

[90] R. Kaminski, L. Lesniak and K. Rybicki, Z. Phys. C 74 (1997) 79. 
[91] D. Cohen, D. S. Ayres, R. Diebold, S. L. Kramer, A. J. Pawlicki, and A. B. Wicklund, Phys. ReV. D 22 (1980) 2595 .

[92] A. D. Martin and E. N. Ozmutlu, Nucl. Phys. B 158 (1979) 520.

[93] A. Etkin et al., Phys. Rev. D 25 (1982) 1786.

[94] A. Mercer, et al., Nucl. Phys. B 32 (1971) 381.

[95] P. Estabrooks, R. K. Carnegie, A. D. Martin, W. M. Dunwoodie, T. A. Lasinski and D. W. G. S. Leith, Nucl. Phys. B 133 (1978) 490.

[96] H. H. Bingham, et al., Nucl. Phys. B 41 (1972) 1.

[97] D. Aston, et al., Nucl. Phys. B 296 (1988) 493.

[98] T. A. Armstrong, et al., Z. Phys. C 52 (1991) 389.

[99] W. Hoogland, et al., Nucl. Phys. B 126 (1977) 109.

[100] M. J. Losty, V. Chaloupka, A. Ferrando, L. Montanet, E. Paul, D. Yaffe, A. Zieminski, J. Alitti, B. Gandois and J. Louie, Nucl. Phys. B 69 (1974) 185.

[101] A. M. Bakker, et al., Nucl. Phys. B 24 (1970) 211.

[102] Y. Cho, et al., Phys. Lett. B 32 (1970) 409.

[103] S. J. Lindenbaum and R. S. Longacre, Phys. Lett. B 274 (1992) 492.

[104] P. Estabrooks and A. D. Martin, Nucl. Phys. B 79 (1974) 301.

[105] G. Ecker and C. Zauner, Eur. Phys. J. C 52 (2007) 315.

[106] J. Ruiz de Elvira, J. R. Pelaez, M. R. Pennington and D. J. Wilson, AIP Conf. Proc. 1257 (2010) 467.

[107] G. P. Lepage and S. J. Brodsky, Phys. Rev. D 22 (1980) 2157.

[108] D. Jido, J. A. Oller, E. Oset, A. Ramos and U. G. Meissner, Nucl. Phys. A 725 (2003) 181.

[109] J. A. Oller, Nucl. Phys. A 727 (2003) 353.

[110] A. Pich, arXiv:hep-ph/0205030.

[111] V. Cirigliano, G. Ecker, M. Eidemuller, R. Kaiser, A. Pich and J. Portoles, JHEP 0504 (2005) 006.

[112] A. Pich, I. Rosell and J.J. Sanz-Cillero, JHEP 1102 (2011) 109.

[113] Z.-H. Guo and P. Roig, Phys. Rev. D 82 (2010) 113016.

[114] H. Georgi, Weak interactions and modern particle theory. Dover Publications, Inc., Mineola, New York, 2009.

[115] K. Kawarabayashi and M. Suzuki, Phys. Rev. Lett. 16 (1966) 255; Riazuddin and Fayyazuddin, Phys. Rev. 147 (1966) 1071.

[116] R.S. Chivukula, E.H. Simmons, H.-J. He, M. Kurachi and M. Tanabashi, Phys. Rev. D 75 (2007) 035005.

[117] J. A. Oller and E. Oset, Nucl. Phys. A 629 (1998) 739.

[118] A. Dobado and J. R. Pelaez, Phys. Rev. D 65 (2002) 077502.

[119] J. R. Pelaez, hep-ph/0509284.

[120] P. Herrera-Siklody, J. I. Latorre, P. Pascual and J. Taron Nucl. Phys. B 497 (1997) 345. 